\title{
Applications of the unsteady vortex-lattice method in aircraft aeroelasticity and flight dynamics
}

\author{
Joseba Murua a, Rafael Palacios b,*, J. Michael R. Graham ${ }^{\mathrm{b}}$ \\ ${ }^{a}$ Division of Mechanical, Medical and Aerospace Engineering, University of Surrey, Guildford GU2 7XH, United Kingdom \\ ${ }^{\mathrm{b}}$ Department of Aeronautics, Imperial College, London SW7 2AZ, United Kingdom
}

\section{A R T I C L E I N F O}

Available online 15 August 2012

\section{Keywords:}

Potential flow

Unsteady aerodynamics

Free-wake models

Aeroelasticity

Flight dynamics

\begin{abstract}
A B S T R A C T
The unsteady vortex-lattice method provides a medium-fidelity tool for the prediction of nonstationary aerodynamic loads in low-speed, but high-Reynolds-number, attached flow conditions. Despite a proven track record in applications where free-wake modelling is critical, other lesscomputationally expensive potential-flow models, such as the doublet-lattice method and strip theory, have long been favoured in fixed-wing aircraft aeroelasticity and flight dynamics. This paper presents how the unsteady vortex-lattice method can be implemented as an enhanced alternative to those techniques for diverse situations that arise in flexible-aircraft dynamics. A historical review of the methodology is included, with latest developments and practical applications. Different formulations of the aerodynamic equations are outlined, and they are integrated with a nonlinear beam model for the full description of the dynamics of a free-flying flexible vehicle. Nonlinear time-marching solutions capture large wing excursions and wake roll-up, and the linearisation of the equations lends itself to a seamless, monolithic state-space assembly, particularly convenient for stability analysis and flight control system design. The numerical studies emphasise scenarios where the unsteady vortex-lattice method can provide an advantage over other state-of-the-art approaches. Examples of this include unsteady aerodynamics in vehicles with coupled aeroelasticity and flight dynamics, and in lifting surfaces undergoing complex kinematics, large deformations, or in-plane motions. Geometric nonlinearities are shown to play an instrumental, and often counter-intuitive, role in the aircraft dynamics. The unsteady vortex-lattice method is unveiled as a remarkable tool that can successfully incorporate all those effects in the unsteady aerodynamics modelling.
\end{abstract}

(c) 2012 Elsevier Ltd. All rights reserved.

\section{Contents}

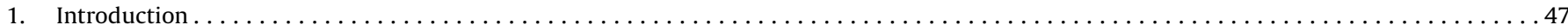

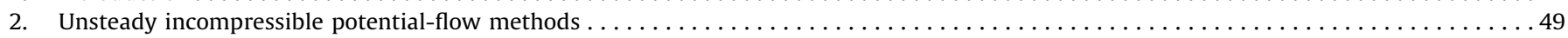

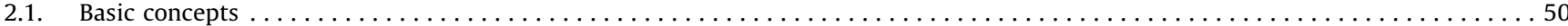

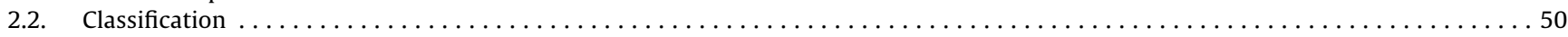

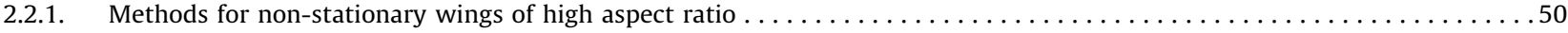

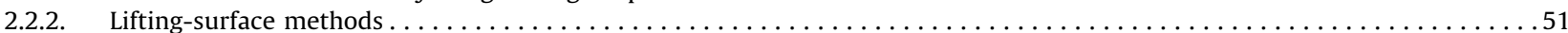

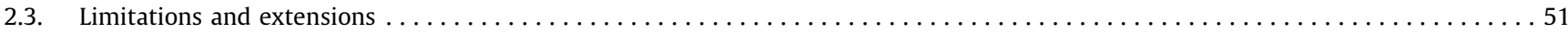

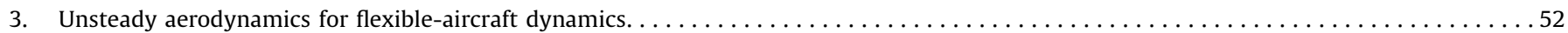

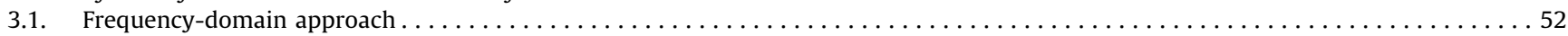

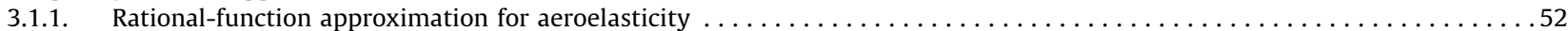

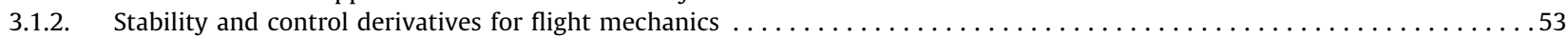

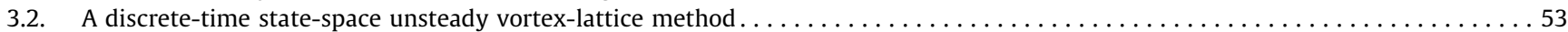

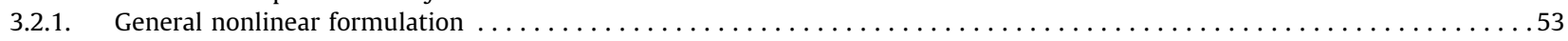

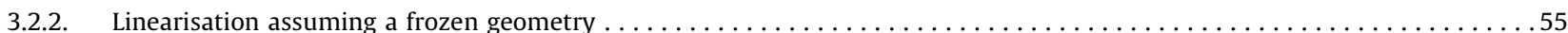

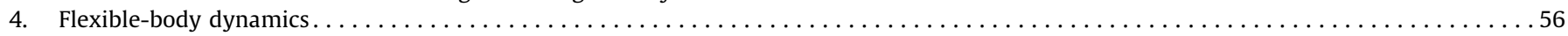

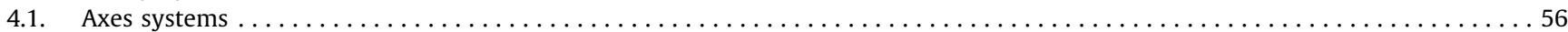

\footnotetext{
* Corresponding author. Room 355, Roderic Hill Building, South Kensington Campus, United Kingdom. Tel.: +44 2075945075.

E-mail address: rpalacio@imperial.ac.uk (R. Palacios).
} 


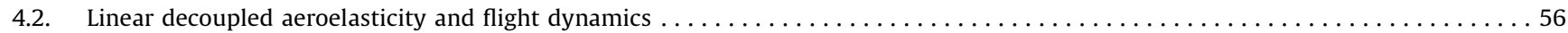

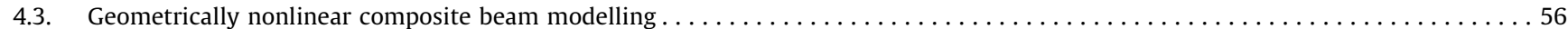

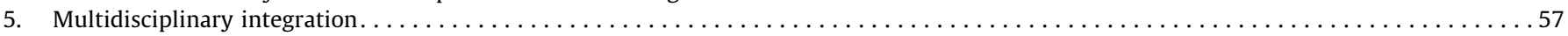

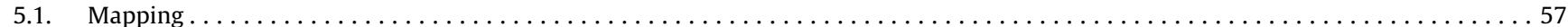

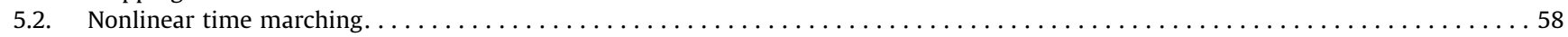

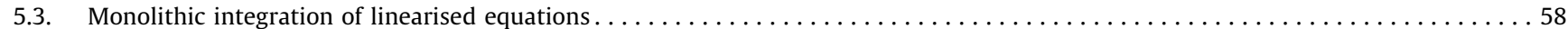

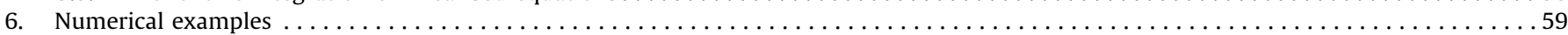

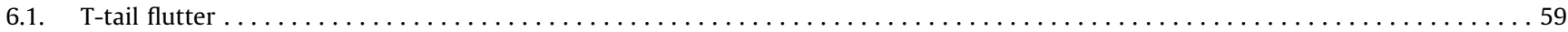

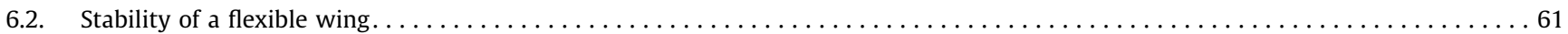

6.3. Linear stability analysis of an aircraft with coupled flight dynamics and aeroelastic response $\ldots \ldots \ldots \ldots$

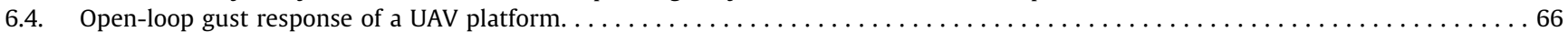

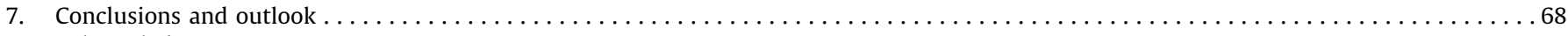

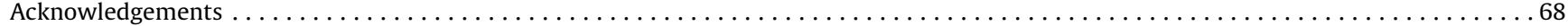

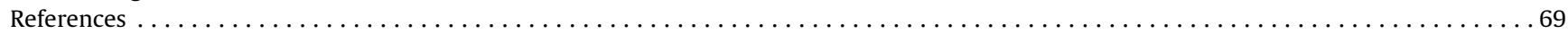

\section{Introduction}

Unsteady aerodynamics based on potential-flow theory has long furnished the basic foundation in low-speed aircraft aeroelasticity and dynamic-load analysis. The doublet-lattice method [1], in particular, has long been the fundamental tool of the aeroelastic community, and has provided a robust approach for non-stationary aerodynamic prediction, although simpler approaches based on strip theory and indicial functions are still used at the conceptual level [2]. However, the applicability of the existing design tools is being stretched as novel concepts are developed and conventional vehicles see their wing stiffness substantially reduced. Aircraft with coupled aeroelasticity and flight dynamics, and lifting surfaces undergoing complex kinematic motions or large deformations are becoming customary, requiring a new paradigm for modelling, analysis, and control synthesis. The unsteady vortex-lattice method, an aerodynamic model with a long and successful track record, can be reengineered to provide again an advantageous alternative in many of these scenarios.

The surge in the number of high-altitude long-endurance (HALE) aircraft, including extreme cases such as solar-powered platforms, attests some of the changes aviation is experiencing. These unmanned aerial vehicles (UAV) are not subject to many traditional restrictions on their overall configuration, and they usually consist of very light, slender and thus flexible structures. This may cause geometrically nonlinear deformations during normal operation and overlap of the aircraft's aeroelastic and flight dynamics natural frequencies, which, in turn, makes them particularly vulnerable to atmospheric disturbances. As a result, any successful modelling effort depends upon a multidisciplinary outlook that integrates aeroelasticity, flight dynamics and controls into a common framework [3,4].

A substantial research effort has been carried out in recent years towards this goal, and even though other alternatives have been explored [5-8], in most cases the presence of a characteristic dominant dimension in the primary flexible structures has motivated descriptions of the nonlinear structural dynamics through composite beam models and of the non-stationary aerodynamics by means of 2D strip theory [4,9-16]. This computationally inexpensive approach has led to the identification of critical phenomena in the behaviour of these vehicles, but neglects relevant 3D flow physics, such as the accurate prediction of wing-tip effects [17], and the aerodynamic interference between wakes and lifting surfaces [18]. Solution of the Navier-Stokes equations for 3D, unsteady flow-fields with large motions of the solid boundaries is still very demanding, not least because of the small meshes needed for boundary-layer resolution. Euler codes are more efficient, but they are also hindered by the requirement of creating and distorting the mesh as the geometrically nonlinear structure deforms. These methods have therefore found very limited applications so far in aircraft aeroelasticity and flight dynamics with large wing deformations [19-21].

At low subsonic speeds, 3D unsteady potential-flow methods provide excellent tools for aerodynamic analysis: without incurring excessive computational costs, they incorporate 3D effects, interference and wake modelling. These methods, however, are not appropriate when the wing enters stalled conditions, to predict viscous drag, or at low Reynolds number. Neither are they adequate at very high altitudes, potentially within the range of operations of HALE UAVs, due to dominance of rarefied flow effects. Note however that the critical load conditions will occur during climb and descent operations in the lower atmosphere, which is the scope of aeroelastic analysis.

The equation governing low-speed potential flows is Laplace's equation for the velocity potential. One of the key features of this linear differential equation is that a 3D flow-field problem can be converted to a reduced-dimension equivalent one by distributing singularity (elementary) solutions over the surface where the flow potential must be found (boundary-value problem). As a result, the numerical solution in terms of singularities is faster compared to field methods where the unknown quantities are distributed in the entire volume surrounding the body-although it must be also acknowledged that whereas the former requires the inversion of a fully populated matrix, the latter results in a sparsely banded one. The reduction of the $3 \mathrm{D}$ computational domain to a surface problem has led to the rapid development of computer codes for the implementation of potential-flow methods, and thanks to their flexibility and relative economy they continue to be widely used despite the availability of more exact approaches.

The foundations of potential-flow vortex-lattice methods (VLM) can be traced back to Helmholtz's seminal work on vortex flows and Joukowski's contributions on circulation [22], but the earliest formulations appeared in the 1930s. Rosenhead [23] studied a 2D vortex-layer by replacing it with a system of vortex filaments. He showed that the vortex sheet rolled up with time, and the rationale behind it was given by Lavrent'ev [24]. The term "vortex lattice" was coined by Falkner in 1943 [25]. The concept was simple but it relied on a numerical solution, so it was not until digital computers became available that practical implementations became widespread. In the meantime, other liftingsurface methods were also pursued, such as the kernel-function method [26], but its development was hindered by the fact that it required a priori knowledge of appropriate pressure modes, and was therefore configuration dependent [27-31].

Hedman [32] established the now classical (steady) VLM in 1965: he idealised the mean aerodynamic surface into small trapezoidal lifting elements each containing a horseshoe vortex with its bound 


\begin{tabular}{|c|c|c|c|}
\hline \multicolumn{2}{|c|}{ Nomenclature } & $\Psi$ & local Cartesian Rotation Vector (CRV) along the beam \\
\hline \multicolumn{2}{|c|}{ Symbols } & $\vec{\omega}$ & $\begin{array}{l}\text { reference line } \\
\text { inertial angular velocity of the body-fixed frame, } a\end{array}$ \\
\hline A & aerodynamic influence coefficient matrix & $\vec{\Omega}$ & inertial angular velocity at a beam location \\
\hline AoA & angle of attack & \multirow{3}{*}{\multicolumn{2}{|c|}{ Subscripts }} \\
\hline C & wake-convection matrix & & \\
\hline$C^{B a}$ & coordinate transformation matrix, from $a$ to $B$ & & \\
\hline$k$ & reduced frequency & $A$ & aerodynamic \\
\hline$Q$ & global column vector of generalised forces & $a$ & body-fixed reference frame \\
\hline$\vec{R}$ & local position vector along the beam reference line & $B$ & deformed (material) reference frame \\
\hline$t$ & physical time & $b$ & bound, corresponding to lifting surface \\
\hline$T$ & thrust per propeller & $c$ & control \\
\hline$\vec{v}$ & $\begin{array}{l}\text { inertial translational velocity of the body-fixed } \\
\text { frame, } a\end{array}$ & $\begin{array}{l}d \\
f\end{array}$ & $\begin{array}{l}\text { exogenous disturbance } \\
\text { flutter }\end{array}$ \\
\hline $\boldsymbol{w}$ & $\begin{array}{l}\text { column vector of velocities induced by the motions } \\
\text { of the lifting surfaces and the incident flow }\end{array}$ & $\begin{array}{l}g \\
F B\end{array}$ & $\begin{array}{l}\text { gust } \\
\text { flexible body, including rigid-body and } \\
\text { structural states }\end{array}$ \\
\hline$u$ & $\begin{array}{l}\text { input vector } \\
\text { ing }\end{array}$ & $k$ & vortex-ring counter \\
\hline$x$ & state vector & $n c$ & non-circulatory \\
\hline$y$ & output vector & $o$ & $\begin{array}{l}\text { tensor evaluated at reference (equilibrium) } \\
\text { conditions }\end{array}$ \\
\hline & & $R$ & rigid-body \\
\hline Greek & & $S$ & structural \\
\hline & & $w$ & wake \\
\hline$\delta$ & $\begin{array}{l}\text { circulation strength of a vortex ring } \\
\text { elevator deflection }\end{array}$ & $\infty$ & free-stream conditions \\
\hline$\zeta$ & coordinates of the aerodynamic lattice & & \\
\hline $\boldsymbol{\eta}$ & $\begin{array}{l}\text { vector of displacements and rotations at all finite } \\
\text { element nodes }\end{array}$ & & \\
\hline$\Theta$ & Euler angles & $\bullet{ }^{n}$ & time step $n$ \\
\hline$v$ & column vector of translational and angular velocities & $\bullet$ & $\begin{array}{l}\text { matrix or vector evaluated at reference (equilibrium) } \\
\text { conditions }\end{array}$ \\
\hline$\vec{\xi}$ & $\begin{array}{l}\text { relative position vector within a beam } \\
\text { section/aerofoil }\end{array}$ & & $\begin{array}{l}\text { time derivatives, } d / d t \\
\text { cross-product operator }\end{array}$ \\
\hline
\end{tabular}

spanwise element along the swept quarter-chord of the element, and locating the collocation points for the non-penetration boundary condition at the three-quarter chord. The downwash at each collocation point was computed through the Biot-Savart law, and compressibility was accounted for by means of the Prandtl-Glauert transformation. The method continued to be widely used in the following decades, as exemplified by the workshop held at NASA Langley in 1976 [33], and alongside different implementations of the code [34-36] various relevant numerical issues were also addressed: increasing convergence and accuracy [37-41], accounting for wake roll-up instead of assuming a flat wake [42], and modelling leadingedge separation [43-47]. The steady VLM has since been employed in a number of applications, such as modelling yacht sails [48], in the computation of stability derivatives and flight dynamics analysis [49-51], in aerodynamic interference of aircraft [52-54], or in multidisciplinary optimisation [55], among many others.

As the VLM was initially limited to steady load calculations it was natural to develop an unsteady equivalent. Albano and Rodden [1] extended the VLM to harmonically oscillating surfaces for an assumed flat wake. Replacing the vortex sheet by one of (equivalent) oscillating doublets, the doublet-lattice method (DLM) was obtained. Indeed, it reduces to the original VLM at zero reduced frequency [56]. The DLM underwent further refinements conducted mainly by Rodden and different collaborators [56-59], to become broadly used for unsteady load computations and the prevalent tool in subsonic aircraft aeroelasticity [60].

When referring to the vortex-lattice method, it is a common misconception (particularly within the aeroelastic community) to assume that it is limited to the steady equivalent of the doublet-lattice method. While this is true for the steady version, it is also known [61] that a panel with a piecewise constant doublet distribution is equivalent to a vortex ring around its periphery. Hence, the VLM can be directly extended to nonstationary situations, giving rise to the time-domain unsteady vortex-lattice method (UVLM). The UVLM is mentioned in many textbooks on aerodynamics, but the most comprehensive description is possibly given by Katz and Plotkin [62, Section 13.12].

Interestingly, the extension of the VLM into the unsteady aerodynamic regime was mainly driven by a viscous phenomenon, namely the need to model the leading-edge separation on delta wings. The pioneering works in the development of the UVLM were carried out by Belotserkovskii [63], Rehbach [64], and researchers at Virginia Tech $[65,66]$ and Technion $[67,68]$.

As opposed to the DLM, which is written in the frequency domain on a fixed geometry, the UVLM is formulated in the time domain and allows the shape of the force-free wake to be obtained as part of the solution procedure. The DLM offers a faster way of computing unsteady aerodynamic loads, but it is a linear method restricted to small out-of-plane harmonic motions with a flat wake. Hence, while the DLM has dominated in fixedwing aircraft aeroelasticity, the UVLM has been gaining ground in situations where free-wake methods become a necessity because of geometric complexity, such as flapping-wing kinematics [69-71], rotorcraft [72,73], or wind turbines [74-80]. With the advent of novel vehicle configurations and increased structural flexibility for which the underlying assumptions of the DLM 
no longer hold, the UVLM constitutes an attractive solution for aircraft dynamics problems and has been recently exercised in problems such as unsteady interference [53,81], computation of stability derivatives [82], flutter suppression [83], gust response $[8,84]$, optimisation [85], morphing vehicles [86], and coupled aeroelasticity and flight dynamics [18].

The UVLM is suitable for quick-turnaround single-processor simulations, and as the computation of each induced velocity is independent of the rest, parallelisation of the code is straightforward. However, the method can still be constrained by computational power for large enough problems: if $N$ is the number of bound and wake elements, the total number of operations per time step goes as $\mathcal{O}\left(N^{2}\right)$. Several procedures have been devised to speed up the solution process. A first possibility is linearisation of the problem. This was carried out by Hall [87], who transformed the UVLM equations into discrete state-space form. Its dominant eigenvalues would define a reduced-order model to, for instance, obtain dynamic aeroelastic stability characteristics. These ideas were explored further in Refs. [88-91]. Another approach is to target the number of evaluations of the Biot-Savart law, trying to reduce it while retaining the accuracy and fidelity of the flow field. In general, the underlying principle consists of separating vortex elements into "near field" and "far field", and making use of the diminishing influence as the distance between the point of evaluation and the vortex elements increases. The simplest alternative is to define a threshold beyond which the calculations are not performed, while retaining the influence over neighbouring elements (this could also include a wake truncation, but in this case the source of error introduced by ignoring the effect of far-field elements over their own near-field propagates and this should be done with care). A more systematic approach is to treat the effects of grouped far-field elements together, for which different alternatives exist, such as sub-grid schemes [92] or fast multipole expansions [70,93]. The latter is derived from the general solution to the n-body problem and reduces the number of operations per time step to $\mathcal{O}(N \log N)$. Other ideas have also been investigated to decrease computational costs, e.g., in helicopter applications. For example, Bagai and Leishman [94] tackled the problem through adaptive refinement of the wake grid, with interpolation of known information onto intermediate points. For a brief introduction about other computation acceleration methods proposed in the rotorcraft community, see Ref. [95, p. 617].

Its repeated evaluation is not the only issue concerning the Biot-Savart law. The use of discrete vortex-segments to account for the vorticity in the shed wake originates numerical difficulties due to the singularity at the vortex filament: The induced velocities at the segment itself and in its neighbourhood can be unphysically large, thereby leading to unrealistic circulation values. This may occur, for example, when a wake encounters a lifting surface or when the wake undergoes an intense roll-up causing crossings of vortex segments, making it difficult to distinguish between the known physical wake instabilities and those of numerical origin [96]. These cases require regularisation (desingularisation), which is extensively described, for instance, in Refs. [92,97-99]. Techniques such as introducing vortex-core models [100-103], using the sub-vortex technique [104], implementing distributed vorticity elements [105], or discarding wake connectivity (the "vortex-blob" method) [106-109] are some of the solutions.

Finally, it is worth pointing out the need to enforce the KuttaJoukowski condition (often shortened as the Kutta condition), necessary to guarantee the uniqueness of the solution in unsteady potential flow-fields, in addition to flow-tangency on impermeable surfaces. The Kutta-Joukowski condition is based on physical considerations, and states that the flow leaves the trailing edge of the aerofoil or wing smoothly, defining the shedding location for an infinitesimally thin wake in which all vorticity of the otherwise irrotational fluid is concentrated. The pressure difference across this sheet is zero. While in potential flows viscosity is neglected in the momentum conservation equations, the Kutta-Joukowski condition permits some aspects of viscous effects to be incorporated. It is well established for steady, attached flow situations, but its validity for time-dependent cases is controversial. Despite being applied in most numerical studies of unsteady wing-theory, in reality it may not hold for highly unsteady flows. Experimental results differ, and there is no definitive conclusion on the reduced-frequency threshold for its validity. It is however safe to assume that for reduced frequencies of $k<0.6$ the KuttaJoukowski condition is satisfied. For further discussion, see Refs. [97,98,110-116].

While Ref. [62] constitutes the fundamental reference for both the theory and numerical implementation of the UVLM, this paper will focus on the use of the UVLM in aeroelasticity and flight dynamics modelling of flexible aircraft. As a result, the paper will try to give some insight into current industrial practice, identifying alternative solution methods, and accentuating situations where the UVLM is particularly advantageous. The paper is therefore organised as follows:

Potential-flow methods for unsteady aerodynamic modelling are reviewed first in Section 2, including classification and limitations, as well as brief descriptions of the most relevant models for unsteady aerodynamic load calculations. The strengths and weaknesses of each option are highlighted. Section 3 compares 3D unsteady aerodynamic models in the context of full aircraft analysis. The conventional methods in aeroelasticity and flight dynamics are surveyed, and a state-space formulation of the UVLM is presented. The benefits and shortcomings of these two methodologies are underlined. Section 4 presents a quick overview of the modelling techniques for flexible-body dynamics. Conventional linear and decoupled analyses are compared to a nonlinear geometrically exact beam model, in which structural and rigid-body degrees of freedom are included. Once the necessary tools for flexible-aircraft dynamic analysis have been outlined, Section 5 details the different possibilities for integration of the separate disciplines into a unified framework for coupled aeroelasticity, flight dynamics, and control. Weakly (loosely) or strongly (tightly) coupling the UVLM with the flexible-body equations permits fully nonlinear time-marching simulations, whereas the linearisation of the equations leads to a very powerful monolithic discrete-time state-space formulation. The latter can be used for stability analysis by solving an eigenvalue problem, in control synthesis for gust alleviation, or can be marched in time. In Section 6, both linear and nonlinear implementations will be exercised in diverse situations in order to illustrate some of the problems this methodology can deal with, and the paper will be concluded in Section 7 by summarising the main merits of the UVLM, and outlining recommendations for the unsteady-aerodynamic modelling of flexible-aircraft dynamics.

\section{Unsteady incompressible potential-flow methods}

Potential theory is an elegant, well developed, and widely used concept in many branches of physics. In the context of fluid dynamics, it constitutes the foundation of low-speed aerodynamics, and, as such, it is described in detail in most textbooks [62,117-119]. It is thus not the aim of this section to give a thorough mathematical derivation of potential-flow theory, but just a quick overview of the basic ideas as they apply to lowspeed aerodynamics, including classification and limitations, and 
to review the most common numerical solution methods employed for aircraft dynamic analysis.

\subsection{Basic concepts}

Inviscid, incompressible, and irrotational flows are governed by Laplace's equation for the velocity potential, $\Phi$

$\nabla^{2} \Phi=0$.

This is a linear and elliptic partial differential equation, which results directly from the continuity equation of incompressible potential flows, and its solutions must be subject to a pair of boundary conditions. For many problems of aeronautical interest these consist of a boundary condition on the body surface enforcing flow tangency for impermeable surfaces, and a boundary condition at infinity. The latter requires that disturbances vanish in the far field. Whereas exact 2D analytical solutions of this boundary-value problem can be obtained for simple geometries by means of conformal transformations, the common procedure in modern aerodynamics is to compute approximate solutions numerically using the boundary-element method, i.e., applying Green's function theory. This approach, which unlike transformation theory, is applicable to $3 \mathrm{D}$ as well as 2D flows, is based on identifying elementary solutions (or singularities), which are algebraic functions of unknown strength that satisfy the linear differential equation. They can be then combined to represent the actual flow field through the superposition principle by distributing them over a surface. As a result, the problem of finding a solution to this partial differential equation over a $3 D$ domain is replaced by the problem of solving a set of algebraic equations for the unknown singularity distribution over the body surface, leading to much faster numerical algorithms. Singularity solutions automatically satisfy the far-field boundary-condition since the velocity fields they induce decay with distance. Their strength is determined by applying the non-penetration boundary condition (flow tangency) at a number of control points on the surface. The resulting system is undetermined with respect to the circulation around the body, and the Kutta-Joukowski condition needs to be imposed to obtain a unique solution.

\subsection{Classification}

Incompressible potential-flow methods can be categorised according to various criteria. Whether the flow is steady or unsteady is an obvious distinction, and this paper will concentrate on unsteady methods. Laplace's equation is time-independent, but temporal dependence is introduced through the boundary conditions. Although the equation is linear, nonlinearity can enter the problem through the geometry. This may occur through displacement of the body surface when this cannot be reduced to a uniform translation or rotation, such as flexible wing and bodies, or through evaluation of boundary conditions on a free wake.

A logical classification of these methods is based on the type of singularity used for the discretisation of the domain: the most familiar elementary solutions (2D and 3D) are sources, doublets and vortices, but computer codes with different combinations of them are also widespread. The so-called higher-order panel methods use singularity distributions that are not constant on the panel, and may also use elements which are non-planar. In theory, for subsonic cases, good results can be obtained using far fewer panels with higher-order methods. In practice, the need to resolve geometric details often leads to the requirement of small elements, and the advantages of higher-order elements are not necessarily obtained. There seems to be some consensus that loworder methods are faster and cheaper $[62,120]$ and that few highorder codes have proved cost-effective [92].
Wake modelling is an essential part in unsteady aerodynamics, since the wake is responsible for the delay in the development of aerodynamic forces, namely the aerodynamic lags. Quasi-steady approximations to the real unsteady flow rely on the assumption that these lags are negligible and that aerodynamic forces would reach instantaneously their steady-state value. In reality, the wake shed by a lifting surface in relative motion to the surrounding fluid is force free, and hence it moves following the local flow velocity. Therefore, incompressible potential-flow methods can also be divided according to the representation of the wake: free-wake models capture this behaviour by obtaining the shape of the wake as part of the solution procedure. This is a computationally expensive process, since it needs the evaluation of the local velocities at the wake grid-points and it is a common simplification to neglect this effect and to consider a prescribed wake shape. This can be determined through empirical observations, such as flow visualisations, or by assuming that the wake is convected with the free stream. For small deformations, this leads to a flatwake simplification, which works well in a broad range of scenarios, but is not suitable for complex kinematics or highly loaded wings.

As aforementioned, solutions to Laplace's equation around general body shapes are obtained by means of singularities distributed over panel elements on the body surface. When the body is thin, there is a considerable gain in solution speed to be had by invoking thin-aerofoil or lifting-surface theory, and placing the singularities and the control points at which the body boundary-condition is satisfied either on the camber-line or the chord-line surface. The former is more appropriate if the wing is thin but its camber, twist and/or deflection are large. When thinaerofoil or lifting-surface theory is used, thickness is represented by sources and all effects of local incidence and camber by elements which include circulation. Panel methods that use combinations of sources and doublets/vortices were routinely used for the steady case, and transformed the aeroplane design process [121]. However, the effect of thickness on overall forces such as lift and moment is second order and unless it is very large, around $30 \%$ of the chord [122], its influence is minimal in nonstationary flows. Hence it is usual to omit them in unsteady thinaerofoil or lifting-surface theory.

In thin-aerofoil or lifting-surface theory where boundary conditions including the wake are on a fixed streamwise plane, unsteady flows may be analysed in either the time of frequency domains, and being completely linear, they are the Fourier transforms of one another. When the singularities are on a nonconstant camber surface, and consistent with this a time-varying non-planar wake is modelled, the problem is not fully linear, and only time-domain solutions are valid. This paper will focus on such methods.

These classification criteria are not mutually exclusive. As already stated, some methods combine different singularities for modelling thin surfaces and wings with thickness, or will allow the user to choose between a prescribed or a free wake. As a consequence, there is no universal classification and different authors might resort to different rules.

\subsubsection{Methods for non-stationary wings of high aspect ratio}

Time-dependent thin-wing theory generates three main lengthscales: chord $c$, span $b$, and typical wavelength $\lambda$ of the wake (related to the reduced frequency, $k$, as $\lambda=\pi c / k$ ). Assuming $c \ll b$, one can identify different situations depending of the values of $\lambda$ [123]. ${ }^{1}$

\footnotetext{
${ }^{1}$ Note that very high-frequencies, $\lambda \ll c$, are not considered, since potential-flow theory is not a good approximation in these situations-recall the unsuitability of the Kutta-Joukowski condition in these cases.
} 
Very low frequency, $\lambda \gg b$ : this is the region of validity of Prandtl's classical lifting-line theory. The wing is divided into horseshoe vortices, the streamwise portions of these vortices are aligned with the free stream, and a quasi-steady representation of the vorticity shed into the wake is assumed.

Low frequency, $\lambda=\mathcal{O}(b)$ : this condition corresponds to lowfrequency unsteady lifting-line theories. Such models can be obtained through matched asymptotic expansions of the acceleration potential between an outer limit (defined by fixed span with chord going to zero) and an inner limit (with fixed chord and span going to infinity) [124]. The solution for flexible wings with longitudinal motions was developed by Ahmadi and Widnall [123], with an extension to arbitrary (but slow) wing trajectories due to Wilmott [125]. While this is a very elegant solution that defines Theodorsen's unsteady-aerofoil and Prandtl's lifting-line models as limiting cases, its applicability is limited by the assumption that $\lambda \gg c$ and it has seldom been used. An alternative unsteady lifting theory has been however proposed by Drela [6] which assumes an arbitrary time-domain variation of the circulation along the span and a (flat) convective wake. This is time marched and, once the instantaneous circulation is obtained, section forces are obtained by the unsteady version of Joukowski's theorem. This approach has been used in ASWING, an integrated framework for flexible aircraft dynamic analysis $[6,126]$.

Intermediate frequency, $c<\lambda<b$ : this condition implies resolving all three scales, which in practice requires lifting surface methods (see below).

High frequency, $\lambda=\mathcal{O}(c)$ : this is the range of validity of $2 \mathrm{D}$ unsteady-aerofoil theory. In the limit, the time averaging in the (periodic) wake cancels spanwise effects and wing aerofoils can be analysed independently. The closed-form solutions to several specific cases are well known: impulsive flows [127], step gusts [128], harmonic oscillations [129], and sinusoidal gusts [130] (the last pair is just the Fourier transform of the first two, even though for historical reasons they are normally presented independently). Based on them, different state-space formulations of the unsteady aerodynamics have been proposed [131,132], mainly, by the rotary-wing community. Ref. [133] provides a recent review of 2D unsteady-aerofoil theory.

This approach is typically referred to as strip theory (or bladeelement theory), and it has been extensively used for HALE UAV modelling. Refs. $[4,10,15]$ are based on Peters' finite-state model [132], while some authors (e.g. Ref. [12,134]) have used the indicial method of Leishman [131]. In addition to its simplicity, strip theory presents the advantage that it easily allows for corrections, including semi-empirical stall models [135,136], and steady viscous drag, using software such as XFOIL [137] and JavaFoil [138]. The main disadvantage is in the evaluation of spanwise variations, which may be critical, even for high-aspectratio wings, when large deflections occur [18]. It is customary to include wing-tip effects as a modulation on the amplitude of the unsteady solution based on the spanwise steady lift distribution $[4,10,15]$. This correction, however, is only valid at low frequencies, since the flow becomes strongly two-dimensional (even near the tip) at high frequencies [17]. This limits in practice the applicability of this approach across different time scales for a given aspect ratio. In addition, the unsteady interference between lifting surfaces and wakes (typically between the wake shed by the main wing and the tail) differs significantly from classical quasi-steady approximations even for simple longitudinal manoeuvres [18].

\subsubsection{Lifting-surface methods}

Models to account for the spanwise flow field of finiteaspect-ratio wings are considered next. In order to model lifting effects antisymmetric singularities such as doublets or vortices are needed, distributed over a mean surface instead of the real geometry and thus ignoring thickness. The 3D doublet-lattice method is probably the most widely used method in industrial dynamic aeroelasticity. The bibliography pertaining the DLM is vast, and two notable references are Blair's work in 1994 [139], who gathered all relevant contributions to the DLM up to that date to give a thorough description of the method, and Rodden's view of the historical development in an anecdotal way [140].

In the DLM, the elementary solution to Laplace's equation is the so-called acceleration-potential doublet. Lifting surfaces are divided in trapezoidal panels parallel to the free stream. The DLM is formulated in the frequency domain and the equations are obtained assuming small out-of-plane harmonic motions of the thing wing, and a flat wake. This restricts the applicability of the method. First of all, the boundary conditions are linearised and a prescribed flat wake is assumed, so the method is not applicable when large deformations occur. Second, the DLM is not ideal for situations in which in-plane kinematics play a pivotal role, such as T-tail flutter, and some type of correction is required $[141,142]$. A further limitation is that it is not able to capture all the steady aerodynamic loads required for flight dynamics analysis, and it is typically complemented by additional specific models [7] (e.g., the steady vortex-lattice method). Within its applicability boundaries, the DLM is a very reliable and efficient tool, in part thanks to the experience accumulated during the past few decades and the substantial literature. More than forty years after being devised, it remains as the standard tool for flutter clearance and gust response in industry, and it is at the core of most relevant commercial software for subsonic aeroelasticity $[143,144]$.

In the unsteady vortex-lattice method, lifting surfaces and wakes are discretised using so-called vortex rings, quadrilateral elements composed by discrete vortex segments in a closed loop. The wake vortex-rings are freely convected according to the local flow velocity, developing into a force-free wake. The basics of the explicit UVLM algorithm are thoroughly described in Ref. [62]. As opposed to the DLM, the shape of the wake is not prescribed and the boundary conditions are not necessarily linearised, and can be enforced on the deformed geometry. In addition, general kinematics are allowed, not being restricted to out-of-plane harmonic motions. As a consequence, the UVLM constitutes an advantageous modelling tool when complex and/or large wing motions occur, and when the prescribed-wake assumption does not hold. Nevertheless, the standard method is a time-marching scheme, not so well suited as the DLM in many aeroelastic simulations. An alternative state-space formulation can overcome this limitationsee Section 3.2.

Unsteady lifting-surface methods, such as the DLM and the UVLM, can be directly employed to model thickness by wrapping the elementary solutions around the real boundary [122]. In this case, a closed distribution of the singularities generates a singular influence coefficient matrix when using either Dirichlet or Neumann boundary conditions in 2D, and with Neumann conditions in 3D-which is the only possibility for the latter. This problem can be overcome by various techniques, such as adding the Kutta-Joukowski condition to all rows to desingularise the matrix.

\subsection{Limitations and extensions}

Laplace's equation is an exact representation of incompressible, inviscid, irrotational flow. This implies that rotational regions are confined to thin sheets which can be represented by the singularities. Delimiting exact parameter thresholds for validity of 
the formulation proves more intricate, though. Usually, the incompressibility condition in the steady case is expressed as $M^{2} \ll 1$, and this is extended to the unsteady case as $k^{2} M^{2} \ll 1$, where $M$ is the Mach number, and $k$ is the reduced frequency. Several compressibility corrections exist for inviscid, subsonic, compressible flow, the Prandtl-Glauert rule being the most well known. This correction is normally implemented, for example, in the DLM. As it is based on the linearised velocity potential equation, the Prandtl-Glauert correction is restricted to thin wings and small angles.

Theoretically, a flow can be regarded as inviscid if the Reynolds number is large enough and the flow remains attached. In such cases, viscous phenomena are contained within the boundary layer, outside which the flow can be effectively considered inviscid. For many practical applications the inviscid flow assumption is not suitable. For one, separation will be a prominent feature whenever the local effective incidence is large, such as when fast, large amplitude kinematics take place. Solutions to overcome this have been proposed in the literature, such as semi-empirical models for dynamic stall in 2D aerofoil theory [135,136], or ad hoc models for the UVLM $[8,145]$. Another way to deal with separation is to define additional wake-shedding points apart from those at the trailing edge [146], analogous to the leading-edge separation of delta wings, but this requires a priori (typically, experimental) knowledge of the separation conditions.

Even for high Reynolds numbers the assumption of inviscid flow presents an important shortcoming, as viscous drag cannot be predicted-the D'Alembert paradox. Aeroelasticity and flight dynamics are mostly concerned with lift and induced drag, respectively, but if the viscous drag component is relevant for the analysis, some type of approximation is necessary, such as using static 2D aerofoil data $[80,100,147]$. A more sophisticated approach to viscous modelling would be to couple a potentialflow method with a boundary-layer solver (see Ref. [62, Section 13.12] for an introduction into the topic), but this is far from trivial for unsteady problems.

The estimation of inviscid induced drag also constitutes a challenge in many potential-flow models. Lifting-surface methods present a singularity at the leading edge, due to zero thickness, and the streamwise component of the pressure loading overestimates the induced drag. This can be overcome by taking into account the leading-edge suction. In contrast, methods which place the singularities around the surface of a body of non-zero thickness and compute the surface pressures do not suffer from the leading-edge problem, and the induced drag is readily available.

Awareness of these limitations allows the use of incompressible potential-flow methods (or their extensions) in many fluid dynamics problems. They offer a great insight and provide remarkably accurate results in numerous situations at a fraction of the computational burden of higher fidelity tools.

\section{Unsteady aerodynamics for flexible-aircraft dynamics}

It is deduced from the discussion above, on the one hand, that lifting-line or strip theory have a limited range of applications (both in the geometry and in the wing dynamics), while, on the other hand, surface panel methods that include wing thickness provide little actual modelling advantage for unsteady aerodynamics at subsonic speeds. Consequently, aeroelastic modelling based on potential-flow theory has overwhelmingly relied on lifting-surface methods.

Moreover, the advantages of state-space formulations have long been recognised for aircraft dynamic analysis [148-151]: powerful algebraic tools, root-locus stability, modern control techniques, which simplify the evaluation of transient solutions (dynamic loads, continuous gust response), aeroservoelastic analytical sensitivity derivatives, and load alleviation models. This section describes how both DLM-based (frequency domain) and UVLM-based (time domain) lifting-surface methods are transformed into convenient state-space form.

\subsection{Frequency-domain approach}

The usual procedure for studies on aircraft dynamics relies on the separation of the time scales between elastic and rigid-body modes, effectively decoupling flight mechanics and aeroelastic analyses. Aerodynamic tools have therefore been developed independently to meet the specific requirements in each discipline. From an aeroelasticity point of view, the frequency-domain DLM is very well suited to flutter analysis. The aerodynamic influence coefficients evaluated with this method are then curved-fitted into state-space time-domain models through rational function approximations [60]. For coupled analysis, this is then linked to rigid-body aerodynamics models, which are typically based on an array of techniques to generate aerodynamic lookup tables with stability and control derivatives [152-154]. A brief summary of this process is included next.

\subsubsection{Rational-function approximation for aeroelasticity}

Typical linear aeroelastic analysis using DLM assumes harmonic displacements on the natural vibration modes of the structure (which are obtained from a finite-element model) and evaluates complex-valued aerodynamic influence coefficients (AICs) for a set of tabulated reduced frequencies, $k$, and flight conditions. It is also extended practice to correct those tables using CFD or wind tunnel data $[155,156]$. These AICs relate the generalised aerodynamic forces, $Q_{a}$, to the linear vibration modes of the flexible structure, $\eta$, the control inputs, $u_{c}$, and any exogenous disturbances (i.e., gust velocities), $u_{d}$, as [60]

$Q_{a}(i k)=q \phi^{T}\left[A I C_{\eta}(i k) \eta+A I C_{c}(i k) u_{c}+A I C_{d}(i k) u_{d}\right]$,

where $\phi$ is the structural modal matrix, and $q$ is the dynamic pressure. Frequency domain methods, such as ' $\mathrm{V}$-g' or 'p-k', can be used for stability analysis with AIC matrices interpolated from the tabulated ones. However, it is also desirable to transform frequency-domain aerodynamic forces furnished by the DLM into a set of time-domain equations, in particular for transient gust response. In order to achieve a state-space system, the generalised force coefficient matrices are approximated by means of rational functions, such as [150]

$Q_{a}(p) \approx A_{0}+A_{1} p+A_{2} p^{2}+\sum_{l=1}^{n_{L}} \frac{p}{p+\gamma_{l}} A_{l+2}$,

or

$Q_{a}(p) \approx A_{0}+A_{1} p+A_{2} p^{2}+D(I p-R)^{-1} E p$,

where $p$ is the nondimensional complex Laplace variable, and $\gamma_{l}$ are the so-called aerodynamic lag terms. All the matrix coefficients are real valued. There are two well-known techniques to determine the lag terms (or poles): Roger's approach [157,158] using Padé approximants, Eq. (3), and Karpel's [149] minimumstate method, Eq. (4). These equations can now be easily transformed into state-space expressions in the time domain and can then be appended to the structural dynamics equations, as elucidated in Section 4.2. This methodology typically requires the input of an experienced engineer: wrong selection of lag values may have a big impact on the results. Also, AICs should cover a wide range of reduced frequencies (even those seemingly of no interest) to obtain good results. 


\subsubsection{Stability and control derivatives for flight mechanics}

The AIC matrices defined above need to be augmented with the aerodynamic coefficients required for flight dynamics. These are typically pre-computed for a variety of flight and control states and are tabulated in databases [159]. These lookup tables are generated from data sheets, design formulae, empirical relations, wind-tunnel and flight-test results, computational methods, or different combinations of them. Experimental results may offer higher accuracy, but they tend to be expensive. Unsteady effects are often not included, and steady or quasi-steady potential-flow models are the most common to fill the aerodynamic tables, but higher fidelity CFD simulations are also gaining ground [160,161]. Databases may be vast and must comprise information covering diverse scenarios, such as small perturbations around trim and large dynamic manoeuvres. In order to incorporate flexibility effects in an otherwise rigid aircraft, derivatives and load distribution can be adjusted using flex-to-rigid ratios or flexibility increments determined by linear aeroelastic analysis [162].

The combination of the aeroelastic equations with timedomain unsteady aerodynamics, derived by either Eq. (3) or (4), and the flight dynamics equations with tabulated aerodynamic derivatives, provides a unified framework for aeroelasticity and flight dynamics. This process is demonstrated, for example, in Refs. [7,163,164].

\subsection{A discrete-time state-space unsteady vortex-lattice method}

The unsteady vortex-lattice method (UVLM) is an efficient computational technique to solve 3D potential flow problems about lifting surfaces. Vortex rings are distributed over the mean surface and the non-penetration boundary condition is imposed at a number of collocation points, leading to a system of algebraic equations. Aerodynamic control surfaces are directly modelled by prescribed deflections of trailing edge panels and the corresponding rotation of normal vectors. As the surface moves along its flight path, a force-free wake is obtained as part of the solution procedure, also represented by vortex rings (see Fig. 1).
The UVLM is normally solved using an explicit time-marching algorithm, which is described in excellent detail in Ref. [62]. Here, the general nonlinear governing equations are presented in discrete-time state-space fashion, which is found to be more suitable for multidisciplinary integration and aircraft dynamics modelling. The nonlinearities arise due to the enforcement of the boundary conditions on the deformed geometry and the free-wake model. While this formulation is necessary for fully nonlinear timedomain simulations, the computational cost is relatively high. Hence, the linearised form of the equations is also subsequently derived by performing small perturbation expansions and assuming a frozen aerodynamic geometry, i.e., flow tangency is enforced on the reference configuration and the wake convects with the free-stream velocity. This formulation follows upon the concepts outlined in Ref. [87].

\subsubsection{General nonlinear formulation}

Each lifting surface is discretised in rectilinear vortex rings. The leading segment of the vortex ring is placed on the panel's quarter-chord line and the collocation point is at the threequarter chord line, which falls at the centre of the vortex ring (see Fig. 1).

At discrete time step $n+1$, the vorticity distribution of the bound vortex elements is determined by applying the nonpenetration boundary condition, formulated as

$A_{c b} \boldsymbol{\Gamma}_{b}^{n+1}+A_{c w} \boldsymbol{\Gamma}_{w}^{n+1}+\boldsymbol{w}^{n+1}=0$,

where $\boldsymbol{\Gamma}_{b}$ and $\boldsymbol{\Gamma}_{w}$ are the column vectors with the circulation strengths in the bound and wake vortex-rings, respectively; $A_{c b}=A_{c b}\left(\zeta_{b}^{n+\epsilon}\right)$ and $A_{c w}=A_{c w}\left(\zeta_{b}^{n+\epsilon}, \zeta_{w}^{n+\epsilon}\right)$ are the wing-wing and wing-wake aerodynamic influence coefficient matrices, computed at the collocation points, and $\zeta_{b}$ and $\zeta_{w}$ are the column vectors with the bound and wake grid coordinates. $\boldsymbol{w}$ in Eq. (5) is the column vector of normal components of all velocities except those induced by bound and wake vorticity, which may encompass deployment of control surfaces, gust induced velocities, wing deformations and rigid-body motions, and will be denoted here as

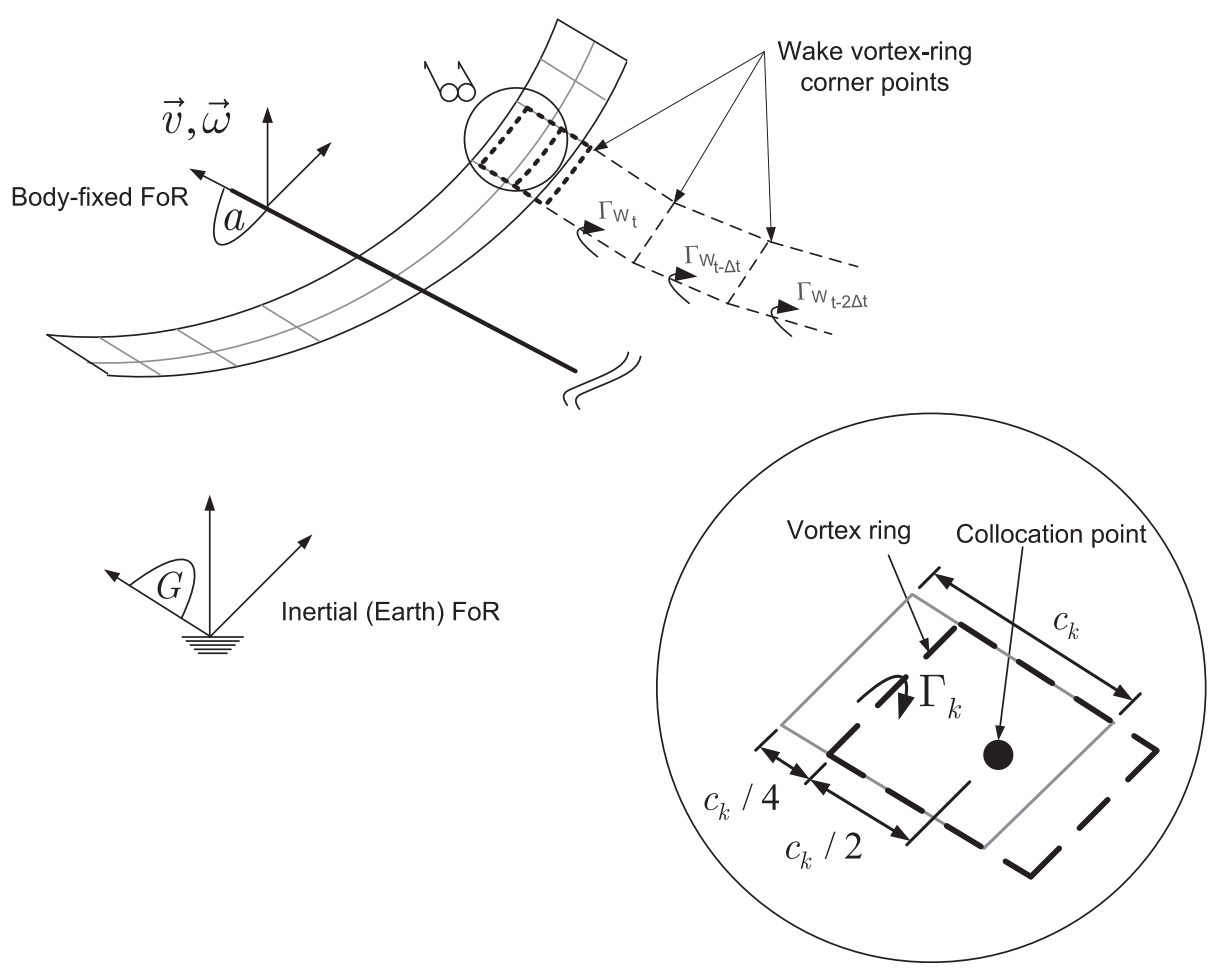

Fig. 1. Unsteady aerodynamics model: lifting-surface and wake discretisation using vortex-ring elements. 
"non-circulatory velocity". The time at which the different variables are evaluated within the current time step is determined by $t^{n+\epsilon}=t^{n}+\epsilon \Delta t$, with $0 \leq \epsilon \leq 1$, and depends on the integration scheme.

The velocity induced by vortex ring $l$ over collocation point $k$ is obtained from the Biot-Savart law, given by (for unit circulation strength)

$\vec{q}_{k l}=\oint_{C_{l}} \frac{d \vec{s}_{l} \times \vec{r}_{k l}}{4 \pi r_{k l}^{3}}$,

where $d \vec{s}$, represents the vortex segments that make up the complete ring, $C_{l}$, and $\vec{r}_{k l}$ is the vector from the collocation point $k$ to the relevant vortex segment of vortex ring $l$, with $r_{k l}$ the corresponding modulus. Numerically, this entails four evaluations of the Biot-Savart law, one for each vortex segment of the closed circuit, $C_{l}$. The elements of the aerodynamic influence coefficient matrices $A_{c b}$ and $A_{c w}$ are obtained by computing all cross-induced velocities at the collocation points and projecting them along the normal vector of the corresponding panel. This can be written as

$\left(A_{c b}\right)_{k l}=\vec{q}_{k l} \vec{n}_{k}, \quad k, l=1 \ldots N_{b}$,

$\left(A_{c w}\right)_{k v}=\vec{q}_{k v} \vec{n}_{k}, \quad k=1 \ldots N_{b}, \quad v=1 \ldots N_{w}$,

where $k, l$ are bound (surface) panel counters, $v$ is a wake vortexring counter, $N_{b}$ is the number of total bound panels, $N_{w}$ the number of wake panels, and $\vec{n}_{k}$ stands for the normal vector of the $k$ th vortex ring (at which the induced velocity is being computed). For numerical efficiency, the adjacent vortex segments shared by different rings need be computed only once. Note that for Eqs. (7)-(8) to be correct, it is necessary to express all magnitudes in the same frame of reference (FoR), and that this needs to be consistent with the other velocity contributions on the non-penetration boundary condition, Eq. (5).

In turn, the velocities induced by the motions of the lifting surfaces and the incident flow, that is, including all contributions save those by bound and wake vorticity (non-circulatory velocity), can be written as

$\boldsymbol{w}^{n+1}=W_{b} \cdot\left(\dot{\zeta}_{b}^{n+1}+\boldsymbol{v}_{d}^{n+1}\right)$

where $\boldsymbol{v}_{d}$ encompasses the effect of exogenous disturbances such as gusts, and $W_{b}\left(\zeta_{b}^{n+\epsilon}\right)$ is a matrix that projects the non-circulatory velocities along the normal direction to the panels-note that defining the normal vector for quadrilateral panels on a surface of double curvature is not straightforward. For a purely aerodynamic problem, the motion of the lifting surfaces will be prescribed and $\zeta_{b}$ and $\dot{\zeta}_{b}$ will be part of the inputs to the system. If a multidisciplinary problem is subject to study, such as coupled aeroelasticity and flight dynamics, $\zeta_{b}$ and $\dot{\zeta}_{b}$ will be a function of other states of the unified model, and they may also encompass other inputs such as deflections of wing control surfaces.

At each time step, as the circulation of the wing changes, a new row of vortex rings will be shed into the wake from the trailing edge of each lifting surface. In addition to this, the existing wake may be displaced following the local flow velocity (the freewake model). This is written as

$\zeta_{w}^{n+1}=C_{\zeta b} \zeta_{b}^{n+1}+C_{\zeta w} \zeta_{w}^{n}+\int_{t^{n}}^{t^{n+1}} \boldsymbol{V}(t) \mathrm{d} t$

where $C_{\zeta b}$ and $C_{\zeta w}$ are very sparse constant convection matrices that update the position of the prescribed wake: $C_{\zeta b}$ closes the newly shed wake panel with the trailing edge of the lifting surface, satisfying the Kutta-Joukowski condition, while $C_{\zeta w}$ preserves the wake of the previous time step unchanged. The column vector $\boldsymbol{V}$ in Eq. (10) includes the local induced flow velocities at the grid points of the wake mesh. If a prescribed wake were to be considered, the integral term would be dropped, but for a fully force-free wake it is necessary to retain it and timeintegration is required to determine the location of the rolled-up wake. Conventionally, this is done using an explicit one-step Euler method, but in order to improve the accuracy and/or stability of the wake roll-up other higher-order schemes have been also proposed in the literature, such as a two-step Euler [165] and the fourth-order Adams-Bashforth-Moulton [73].

These velocities at the vertices of the wake mesh, $\boldsymbol{V}$ in Eq. (10), can be written as

$\boldsymbol{V}=A_{v b} \boldsymbol{\Gamma}_{b}+A_{v w} \boldsymbol{\Gamma}_{w}+\boldsymbol{v}_{\infty}+\boldsymbol{v}_{d}$,

which is analogous to the non-penetration boundary condition, Eq. (5), but in this case the velocities are computed at the wake vertices, and as they are not projected along any vector, the three velocity components are retained-the velocities can also be computed at centres of wake panels or centres of vortex elements, and then interpolated. $\boldsymbol{v}_{\infty}$ represents the velocity due to the freestream conditions, $\boldsymbol{v}_{d}$ the contribution from exogenous disturbances, and the rest of the contributions to the velocity are due to vortex influence, where the entries of matrices $A_{v b}$ and $A_{v w}$ are obtained through the Biot-Savart law, Eq. (6). Note that again, for numerical efficiency, only the velocities at the independent wake nodes need to be computed.

The propagation equation for the wake circulation can be written in discrete time as

$\Gamma_{w}^{n+1}=C_{\Gamma b} \Gamma_{b}^{n}+C_{\Gamma w} \Gamma_{w}^{n}$,

where $C_{\Gamma b}$ and $C_{\Gamma w}$ map the circulation of the previous time step to the current one, and they are very sparse constant matrices which account for Kelvin's circulation theorem (that enforces the condition for wake shedding at the trailing edge) and Helmholtz's vortex theorem (in the convection of the wake). Note that the wake is a thin shear layer subject to Kelvin-Helmholtz instability, which may be captured for fine enough discretisations of the vortex sheet [166] — while the real instability is determined by the thickness of the sheet, the instability in the model is determined by the discretisation, with growth rates increasing as the inverse of the discretisation spacing. As the influence of the wake decreases very rapidly as it is convected away from the lifting surface, Eq. (6), the computational burden can be significantly alleviated by neglecting the influence of remote panels (wake truncation). Implementing dissipation models has also been tried [69,95], but consideration must be given to conservation of circulation issues.

Finally, once the distribution of vorticity has been obtained at each time step, the inviscid aerodynamic loads can be computed. The aerodynamic forces act on the plane defined by the normal vector of the vortex ring and the instantaneous non-circulatory velocity at the collocation point, $\vec{v}_{n c, k}=\left(\vec{\zeta}_{b}+\vec{v}_{d}\right)_{k}$, which encompasses the contributions of rigid-body motions, elastic

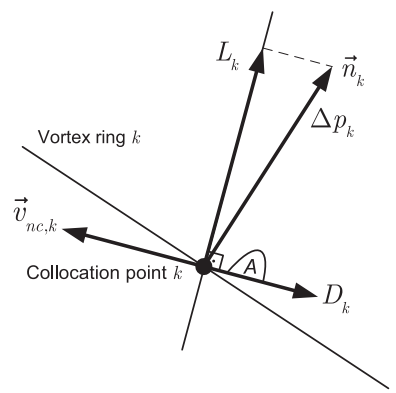

Fig. 2. 2D projection of local frame $A$ of vortex ring $k$, defined by the instantaneous non-circulatory velocity at the collocation point and the normal vector. 
deformations and incident flow-note that if the influence of bound and wake circulation were added, the velocity vector would be tangential to the vortex ring due to the non-penetration boundary condition. This defines a local frame, $A$, as illustrated in the $2 \mathrm{D}$ projection in Fig. 2. The pressure differential, obtained from the unsteady Bernoulli equation, acts along the normal vector. However, as the UVLM is based on thin-wing theory, it does not account for the leading-edge suction [167], and only the component normal to the non-circulatory velocity is retained, i.e., the contribution of pressure to the local lift. The contribution of the panel to the induced drag is aligned with the instantaneous non-circulatory velocity, $\vec{v}_{n c, k}$, and it can be computed through the approximation proposed in Ref. [62]. The vectors storing these inviscid loads are computed using the generalisation of the equations to cater for arbitrary motions not limited to small angles of attack, given in Ref. [71] as

$\boldsymbol{L}^{n}=\rho_{\infty} G_{c}\left[\left(U_{c} \Lambda_{c}+U_{s} \Lambda_{s}\right) \boldsymbol{\Gamma}_{b}^{n}+\dot{\boldsymbol{\Gamma}}_{b}^{n}\right]$,

$\boldsymbol{D}^{n}=\rho_{\infty}\left[-\hat{U} \Lambda_{c} \boldsymbol{\Gamma}_{b}^{n}+G_{s} \dot{\boldsymbol{\Gamma}}_{b}^{n}\right]$,

where $\Lambda_{c(s)}$ are matrices filled with 1 and -1 in the correct positions in order to account for adjacent panels; matrices $G_{c(s)}=$ $G_{c(s)}\left(\zeta_{b}^{n}, \dot{\zeta}_{b}^{n}\right)$ are diagonal matrices dependent on the panel geometry and local angle of incidence; $U_{c(s)}=U_{c(s)}\left(\Gamma_{w}^{n}, \zeta_{b}^{n}, \zeta_{w}^{n}, \dot{\zeta}_{b}^{n}\right)$ and $\hat{U}=\hat{U}\left(\boldsymbol{\Gamma}_{b}^{n}, \boldsymbol{\Gamma}_{w}^{n}, \zeta_{b}^{n}, \zeta_{w}^{n}\right)$ are diagonal matrices that store weighted velocities. Their exact definitions can be found in Ref. [168].

The above UVLM equations can be cast for convenience into general nonlinear discrete state-space form. The propagation expressions, Eqs. (5), (10), and (12), and the output ones, Eqs. (13), can be written, respectively, as

$f_{A}\left(\boldsymbol{x}_{A}^{n+1}, \boldsymbol{u}_{A}^{n+1}\right)=g_{A}\left(\boldsymbol{x}_{A}^{n}, \boldsymbol{u}_{A}^{n}\right)$,

$\boldsymbol{y}_{A}^{n}=h_{A}\left(\boldsymbol{x}_{A}^{n}, \boldsymbol{u}_{A}^{n}\right)$,

where the aerodynamic states and inputs that fully define the aerodynamic system are

$\boldsymbol{x}_{A}=\left\{\begin{array}{c}\boldsymbol{\Gamma}_{b} \\ \boldsymbol{\Gamma}_{w} \\ \dot{\boldsymbol{\Gamma}}_{b} \\ \boldsymbol{\zeta}_{w}\end{array}\right\}$ and $\boldsymbol{u}_{A}=\left\{\begin{array}{l}\zeta_{b} \\ \dot{\zeta}_{b}\end{array}\right\}$.

Note that $\dot{\boldsymbol{\Gamma}}_{b}$ has been included in the state vector since it appears on the aerodynamic loads. It may seem unusual to retain the inputs at the current time step, $\boldsymbol{u}_{A}^{n+1}$, but it will be seen that this allows no loss of generality when integrating the aerodynamic model into a multidisciplinary analysis framework (see Section 5.2).

\subsubsection{Linearisation assuming a frozen geometry}

Eqs. (14) correspond to the general state-space formulation of the UVLM, which can account for large deflections of the lifting surfaces in unsteady flow. In many cases, though, it will be useful to look at the linearised form of these equations, when relative deformations are small around an equilibrium condition, for problems such as stability analysis and design of linear control laws. In this context, the equilibrium condition will generally correspond to a trimmed aircraft configuration, which can exhibit large static deformations. This reference configuration represents the initial conditions for impulsive motions, and the mean conditions for periodic oscillations.

The linearisation of the unsteady aerodynamics, Eqs. (5)-(13) is carried out under the following assumptions:

- Body-fixed axes are used. This is the natural description to obtain the aerodynamic forces, but differs from the usual definition of stability axes used in flight dynamics analysis [152].

- The dynamic excursions around the statically deformed aircraft are small, and as a consequence, the non-penetration boundary condition, Eq. (5), can be enforced at the mean statically-deformed reference geometry. As a result, the dependencies on $\zeta_{b}$ are neglected, except for matrix $W_{b}$, Eq. (9), since this is necessary in order to account for local angle of incidence changes as the lifting surface deforms.

- The application point of the aerodynamic forces does not change as the surface undergoes small deformations and remains constant at the original configuration. Hence, the dependencies on $\zeta_{b}$ are ignored in Eqs. (13). However, the change in orientation of the aerodynamic loads, which depends on the velocities, $\dot{\zeta}_{b}$, is included. This influence appears on matrices $G_{c}$ and $G_{s}$ through the local angle of attack. This approach coincides with the traditional linearisation of aerodynamic forces, where the geometry is assumed frozen but the dependency on velocities is catered for.

- Free-wake effects around the reference condition are neglected. This assumption reduces the UVLM to a prescribed-wake method, and under this approximation it is not necessary to keep track of the wake shape after trim as it conforms to free-stream convection alone. Note that the wake is prescribed in this case, but it does not need to be flat, and it will be shed from the deformed lifting surface. Besides, a rolled-up wake can be considered in order to determine equilibrium more accurately (in a steady sense). As the wake is frozen, there is no need to retain Eq. (10), and $\zeta_{w}$ will remain constant at the reference configuration and is no longer a state of the system. The aerodynamic states that fully define the UVLM are only circulation strength distributions and the derivative of the bound circulation.

Using a mid-point integration scheme for the derivatives of the bound circulations, and performing a small perturbation analysis on the general UVLM equations, the linearised (incremental) propagation equations are obtained as

$A_{c b}^{\mathrm{o}} \Delta \boldsymbol{\Gamma}_{b}^{n+1}+A_{c w}^{\mathrm{o}} \Delta \boldsymbol{\Gamma}_{w}^{n+1}=\left(\frac{\partial W_{b}}{\partial \zeta_{b}}\right)_{\mathrm{o}} \dot{\zeta}_{b}^{\mathrm{o}} \Delta \zeta_{b}^{n+1}+W_{b}^{\mathrm{o}} \Delta \dot{\zeta}_{b}^{n+1}$,

$\Delta \Gamma_{w}^{n+1}=C_{\Gamma b}^{o} \Delta \Gamma_{b}^{n}+C_{\Gamma w}^{0} \Delta \Gamma_{w}^{n}$,

$\Delta \boldsymbol{\Gamma}_{b}^{n+1}-\frac{1}{2} \Delta t \Delta \dot{\boldsymbol{\Gamma}}_{b}^{n+1}=\Delta \boldsymbol{\Gamma}_{b}^{n}+\frac{1}{2} \Delta t \Delta \dot{\boldsymbol{\Gamma}}_{b}^{n}$,

where $\Delta t$ is the time step in the temporal discretisation and "o" defines the reference conditions about which the linearisation takes place and at which matrices and tensors are evaluatedsuperscripts are employed for matrices and column vectors, and subscripts for tensors. Defining $Y=U_{c} \Lambda_{c}+U_{s} \Lambda_{s}$ for conciseness, the linearised output equations for the aerodynamic loads are given by

$$
\begin{aligned}
& \Delta \boldsymbol{L}^{n}=\rho_{\infty}\left\{G_{c}^{\mathrm{o}} \gamma^{\bullet} \Delta \boldsymbol{\Gamma}_{b}^{n}+G_{c}^{\mathrm{o}}\left(\frac{\partial \Upsilon}{\partial \boldsymbol{\Gamma}_{w}}\right)_{\mathrm{o}} \boldsymbol{\Gamma}_{b}^{\mathrm{o}} \Delta \boldsymbol{\Gamma}_{w}^{n}+G_{c}^{\mathrm{o}} \Delta \dot{\boldsymbol{\Gamma}}_{b}^{n}\right. \\
& \left.+\left[G_{c}^{\mathrm{o}}\left(\frac{\partial \Upsilon}{\partial \dot{\zeta}_{b}}\right)_{\mathrm{o}} \boldsymbol{\Gamma}_{b}^{\mathrm{o}}+\left(\frac{\partial G_{c}}{\partial \dot{\boldsymbol{\zeta}}_{b}}\right)_{\mathrm{o}}\left(\Upsilon^{0} \boldsymbol{\Gamma}_{b}^{\mathrm{o}}+\dot{\boldsymbol{\Gamma}}_{b}^{\mathrm{o}}\right)\right] \Delta \dot{\boldsymbol{\zeta}}_{b}^{n}\right\}, \\
& \Delta \boldsymbol{D}^{n}=\rho_{\infty}\left\{-\left[\left(\frac{\partial \hat{U}}{\partial \boldsymbol{\Gamma}_{b}}\right)_{\mathrm{o}} \Lambda_{c} \boldsymbol{\Gamma}_{b}^{o}+\hat{U}^{\mathrm{o}} \Lambda_{c}\right] \Delta \boldsymbol{\Gamma}_{b}^{n}-\left(\frac{\partial \hat{U}}{\partial \boldsymbol{\Gamma}_{w}}\right)_{\mathrm{o}} \Lambda_{c} \boldsymbol{\Gamma}_{b}^{\mathrm{o}} \Delta \boldsymbol{\Gamma}_{w}^{n}\right. \\
& \left.+G_{s}^{o} \Delta \dot{\boldsymbol{\Gamma}}_{b}^{n}+\left(\frac{\partial G_{s}}{\partial \dot{\zeta}_{b}}\right)_{o} \dot{\boldsymbol{\Gamma}}_{b}^{o} \Delta \dot{\zeta}_{b}^{n}\right\} \text {. }
\end{aligned}
$$


The linear discrete-time equations, Eqs. (16)-(20), define the descriptor state-space form of the UVLM. In compact form they are

$E_{A} \Delta \boldsymbol{x}_{A}^{n+1}+F_{A} \Delta \boldsymbol{u}_{A}^{n+1}=A_{A} \Delta \boldsymbol{x}_{A}^{n}+B_{A} \Delta \boldsymbol{u}_{A}^{n}$,

$\Delta \boldsymbol{y}_{A}^{n}=C_{A} \Delta \boldsymbol{x}_{A}^{n}+D_{A} \Delta \boldsymbol{u}_{A}^{n}$,

where the outputs $\boldsymbol{y}_{A}$ are the aerodynamic loads, Eqs. (19)-(20). This descriptor form of the equations, with matrix $E_{A}$ premultiplying the updated value on the state variable is preferred to the canonical discrete-time form $\left(x^{n+1}=A x^{n}\right)$ because this is the natural expression obtained from the UVLM.

The linearised state-space UVLM provides a compact and efficient tool, of comparable fidelity to the DLM, but without some of its restrictions: the wake can be nonplanar, flow tangency is imposed on the statically deformed geometry, and in-plane deformations are captured. Besides, it will be shown in Section 5.3 that it enables the incorporation of rigid-body motions and elastic deformations in a unified monolithic framework.

The UVLM can also replace the DLM in virtually any aeroelastic/flight mechanic analysis. Being a time-domain method, procedures for CFD-based aeroelasticity are directly applicable here (e.g., the Eigensystem Realization Algorithm of Ref. [169]). For flight mechanics applications, the steady vortex-lattice method is already used for the determination of quasi-steady stability and control derivatives [49-51], and implementing the unsteady version of the code would directly incorporate nonstationary effects. Specifically, Eqs. (19)-(20) would provide the variations of the aerodynamic loads with the motions defined by $\zeta_{b}$ and $\dot{\zeta}_{b}$ in order to generate the aerodynamic database.

\section{Flexible-body dynamics}

A small digression is needed at this stage to introduce the elastic and rigid-body equations of motion that are required to describe the dynamics of the flexible vehicle. This section summarises the state-of-the-art in these disciplines.

\subsection{Axes systems}

The choice of axes, both orientation and location, to describe vehicle dynamics has traditionally varied across disciplines. In flight mechanics, it is common to adopt body axes for a Lagrangian description of the motion instead of Earth or ground inertial axes (Eulerian). The motivation to select certain directions and origin lies on the potential to eliminate inertia couplings or to derive stability derivatives more easily. For instance, if the axes are aligned instantaneously with the principal axes, the inertia tensor does not present off-diagonal terms. In turn, stability axes, also known as aerodynamic or wind axes, offer advantages when handling aerodynamic terms.

Regarding the location of the origin of the reference frame, the centre of mass is the norm for a rigid aircraft. However, for a vehicle that undergoes deformations, this approach manifests complications: under dead loads, the centre of gravity of the unsupported structure remains constant (as long as forces are in equilibrium), but its position changes if aerodynamic (i.e., follower) forces are present. Axes systems attached to the (moving) centre of mass of a deformable aircraft define a floating frame [170]. This has led to the mean axes assumption [171,172], which assumes inertia decoupling between the structural-dynamics and rigid-body equations when the latter are referred to the instantaneous centre of mass and principal axes of inertia. While this approach is still used by several authors, the validity of the assumption remains controversial at best [173].

The second, most general, option is therefore to use body-fixed axes at an arbitrary point of the undeformed structure. The origin of the reference frame maintains a constant location on the vehicle and the elastic deformations are referred to it. This does not offer inertial decoupling of elastic and rigid-body degrees of freedom, but sets an appropriate basis for geometrically-exact descriptions $[4,11,17]$.

\subsection{Linear decoupled aeroelasticity and flight dynamics}

As aforementioned, aeroelasticity and flight dynamics can be decoupled for stiff enough vehicles in slow turns (small gyroscopic forces). This is justified in many aeroplanes, and it provides an efficient and adequately accurate way of tackling the dynamics of relatively stiff aircraft. Furthermore, the vehicle structural dynamics can be reduced to a small number of degrees of freedom through modal analysis (based on the free-free modes of the unsupported structure). The existence of mean axes can then be hypothesised, so that the Newton-Euler equations at the centre of gravity of the rigid aircraft can be used to replace the rigid-body modes of the rigid structure. This results in a set of decoupled equations of motion [172]

$$
\begin{aligned}
& \frac{d}{d t}(m \vec{v})=\vec{F}, \\
& \frac{d}{d t}(I \vec{\omega})=\vec{M},
\end{aligned}
$$$$
M_{r} \ddot{\boldsymbol{q}}+C_{r} \dot{\boldsymbol{q}}+K_{r} \boldsymbol{q}=\boldsymbol{Q}_{a},
$$

where $M_{r}, C_{r}$, and $K_{r}$ are the reduced modal inertia, damping, and stiffness matrices, respectively; $m$ is the mass of the aircraft, $I$ is the inertia tensor, $\vec{v}$ and $\vec{\omega}$ are the linear and angular velocities, respectively, and $\vec{F}$ and $\vec{M}$ are the applied forces and moments at the centre of gravity, which may include aerodynamic, propulsive, and gravitational loads. The unsteady aerodynamic loads, $\boldsymbol{Q}_{a}$, are obtained as the corresponding generalised forces for these mode shapes and can be computed from any of the methods defined above. They introduce additional states, either from the lags in the rational function approximation, Eqs. (3) and (4), or from the circulations in the state-space UVLM.

These equations are finally complemented with the propagation equations of the rigid body, which computes the orientation of the vehicle (through, e.g., Euler angles, $\Theta$ ) [153]. For stability analysis, the rigid-body equations are linearised about an equilibrium configuration (trimmed aircraft), and are complemented by the stability derivatives introduced in Section 3.1.2.

\subsection{Geometrically nonlinear composite beam modelling}

For vehicles with larger, more flexible and higher-aspect-ratio wings (e.g., HALE UAVs), geometrically exact beams are considered as a means of incorporating nonlinear effects, and integrating into a unified framework flexible-body dynamics. The primary structures (wing, fuselage, empennage) are modelled as composite curvilinear beams capable of large deflections and global rotations, but under the assumption of small local strains [9,174]. They undergo 3D displacements and rotations, with cross-sectional properties calculated along the span. Typically those displacements and rotations are the primary variables in the numerical solution of the structural problem $[175,176]$, but alternative solutions exists using the strain of the beam elements as primary variables $[4,15,177]$, or taking both the local velocities and strains (the intrinsic description) as independent degrees of freedom [178-180] — the latter can provide some numerical advantages on aircraft-type geometries. A comparative study on these different structural models for flexibleaircraft dynamics was presented in Ref. [17].

Here, nodal displacements, $\vec{R}$, and the cartesian rotation vector (CRV), $\Psi$, are taken as primary degrees of freedom. There are no constraints on the undeformed configuration allowing the 


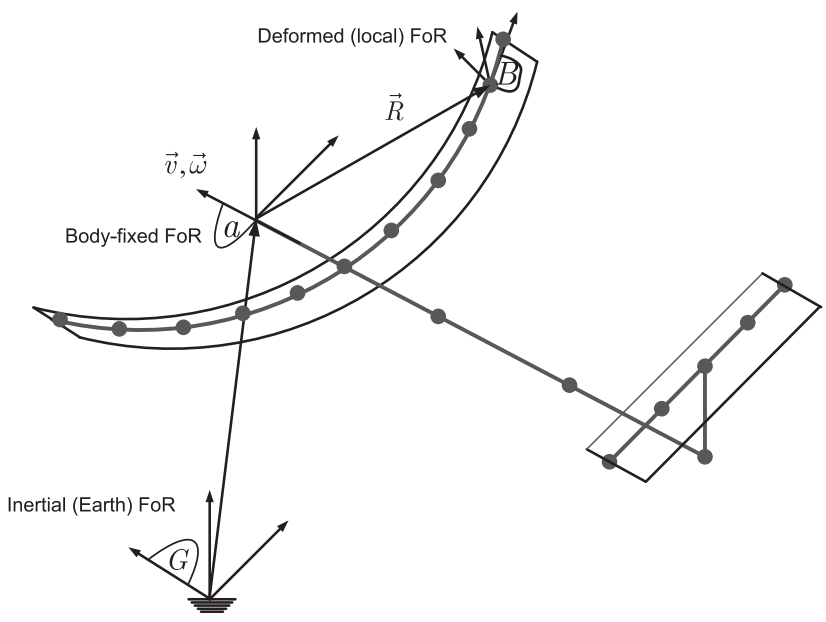

Fig. 3. Flexible-body model: geometrically exact beam elements.

beam to be initially curved and twisted. As shown in Fig. 3, the vehicle dynamics are described by a body-fixed frame of reference (FoR), $a$, which moves with respect to an inertial frame, $G$, by the translational, $v_{a}(t)$, and angular, $\omega_{a}(t)$, velocities of its originsubscripts are used to indicate the coordinate system in which each vector magnitude is projected. The orientation of the body-fixed frame with respect to the inertial one is given by the coordinate transformation matrix $C^{G a}(t)$, determined via Euler angles, $\Theta$, and a flat Earth is assumed. The local orientation of the beam cross sections (aerofoils) is defined by their local coordinate systems, $B$, in the deformed (or current) configuration.

The equations of motion are obtained from Hamilton's principle [17], for which the potential, $\mathcal{U}$, and kinetic, $\mathcal{T}$, energy densities per unit length are first computed as

$\mathcal{U}=\frac{1}{2}\left\{\gamma^{T} \kappa^{T}\right\} \mathcal{S}\left\{\begin{array}{c}\gamma \\ \kappa\end{array}\right\} \quad$ and $\quad \mathcal{T}=\frac{1}{2}\left\{V_{B}^{T} \Omega_{B}^{T}\right\} \mathcal{M}\left\{\begin{array}{c}V_{B} \\ \Omega_{B}\end{array}\right\}$.

Here, $V_{B}$ and $\Omega_{B}$ are the inertial velocities of the local deformed frame, $\gamma$ and $\kappa$ are the beam strains, and $\mathcal{M}$ and $\mathcal{S}$ are the mass and stiffness matrices, respectively, which are obtained through an appropriate cross-sectional analysis methodology [181,182]. The orientation of cross-sections at each point in the current configuration is described in terms of finite rotations from the body-fixed reference frame, $a$, to the local deformed frame, $B$, using the CRV, $\Psi(s, t)$. The corresponding coordinate transformation matrix will be $C^{B a}(\Psi)$. Strains and velocities can then be expressed in terms of the independent set of variables, $R_{a}(s, t)$ and $\Psi(s, t)[174,176]$. This general description of the beam dynamics is independent of any discretisation used. Here, the position and rotation vectors within the $n$-th element are approximated by given shape functions. If $\boldsymbol{\eta}$ is the column vector of all nodal displacements and rotations, $\boldsymbol{\eta}=\left[\begin{array}{ll}\boldsymbol{R}_{a}^{T} \boldsymbol{\Psi}^{T}\end{array}\right]^{T}$, and defining $v=\left[v_{a}^{T} \omega_{a}^{T}\right]^{T}$, the equations of motion of the aircraft can be cast into the following discrete form [17]:

$M(\boldsymbol{\eta})\left\{\begin{array}{c}\ddot{\boldsymbol{\eta}} \\ \dot{v}\end{array}\right\}+Q_{g y r}(\boldsymbol{\eta}, \dot{\boldsymbol{\eta}}, v)+Q_{s t i f}(\boldsymbol{\eta})=Q_{e x t}\left(\boldsymbol{\eta}, \dot{\boldsymbol{\eta}}, v, \Theta, \boldsymbol{u}_{F B}\right)$,

where matrix $M$ is the tangent mass matrix and $Q_{g y r}, Q_{s t i f f}$ and $Q_{e x t}$ are the discrete gyroscopic, stiffness, and external generalised forces, respectively. The input vector $\boldsymbol{u}_{F B}=\left[\boldsymbol{u}_{S}^{T} \mid \boldsymbol{u}_{R}^{T}\right]^{T}$ includes the dependency of the external loads on any other variable in the most general form. In particular, for the aeroelastic and flight dynamics analysis, $\boldsymbol{u}_{F B}$ will depend on the aerodynamic loads, which in turn depend on the aerodynamic states.
The linearised (incremental) form of Eq. (24) around an equilibrium point is given by

$M\left(\boldsymbol{\eta}_{o}\right)\left\{\begin{array}{c}\Delta \ddot{\boldsymbol{\eta}} \\ \Delta \dot{v}\end{array}\right\}+C\left\{\begin{array}{c}\Delta \dot{\boldsymbol{\eta}} \\ \Delta v\end{array}\right\}+K\left\{\begin{array}{c}\Delta \boldsymbol{\eta} \\ 0\end{array}\right\}=\Delta Q_{\text {ext }}$,

where $C$ and $K$ are the tangent damping and stiffness matrices, evaluated at the equilibrium point.

The states that fully determine the flexible-body dynamics are therefore

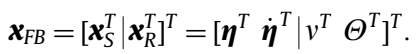

\section{Multidisciplinary integration}

When referring in previous sections to traditional methods for aircraft dynamics, it has been insisted upon that habitually, following the frequency separation approach, aeroelasticity and flight mechanics are studied as independent blocks. The modules are then assembled using interpolation matrices, and adjustments might be made to account for elasticity in the rigidbody equations or vice versa. Assuming that the mean axes assumption holds, this decoupled linear approach reduces the size of computational models and enables straightforward implementation of standard control synthesis techniques.

However, this procedure is not suitable for vehicles that exhibit moderate-to-large wing excursions, and a substantial effort has been recently made to build unified frameworks that incorporate flexible-multibody dynamics and potential-flow aerodynamics $[4,7,10,11,16]$. These have nevertheless been restricted so far to $2 \mathrm{D}$ aerodynamics or linearised boundary conditions.

Coupling the unsteady vortex-lattice method (Section 3.2) with a geometrically nonlinear beam (Section 4.3) overcomes some of the limitations of the above models, and allows fully nonlinear time-marching simulations and linearised monolithic state-space solutions. The mapping between discretisations and different types of integration are presented next, and the reader is referred to Refs. [183,184] for a more general overview of fluid/ structure coupling schemes.

\subsection{Mapping}

As the flexible-body model is based on beams (curves in space) and the aerodynamic lattice is distributed over a lifting surface, a mapping procedure is required between both meshes. This is accomplished here by assuming that the lifting-surface crosssections remain undeformed, that the finite-element discretisation of the beam coincides with the spanwise aerodynamic grid, and that the aerodynamic loads can be approximated as isolated forces acting on the centre of the leading segment of each vortex-ring [18].

First, displacements and rotations of the beam nodes, $\boldsymbol{R}_{a}$ and $\boldsymbol{\Psi}$, and the corresponding rates, $\dot{\boldsymbol{R}}_{a}$ and $\dot{\boldsymbol{\Psi}}$, have to be transformed to deformations and velocities of the grid points of the aerodynamic lattice, $\zeta_{b}$ and $\dot{\zeta}_{b}$. For the vortex-ring vertices, the transformations of the relative position with respect to the body-fixed frame, $\left(\zeta_{b}\right)_{a}$, and inertial velocity expressed in the $a$ frame, $\left(\dot{\zeta}_{b}\right)_{a}$, are given by

$\left(\zeta_{b}\right)_{a}=R_{a}+C^{a B} \xi_{B}$,

$\left(\dot{\zeta}_{b}\right)_{a}=v_{a}+\widetilde{\omega}_{a} R_{a}+\dot{R}_{a}+C^{a B} \widetilde{\Omega}_{B} \xi_{B}$,

where $\xi_{B}$ is the (constant) distance between a vortex-ring corner point and the relevant node, and $\Omega_{B}=T(\Psi) \dot{\Psi}+C^{B a}(\Psi) \omega_{a}$ is the local inertial angular velocity, with $T(\Psi)$ the so-called tangential operator [178]. Note that variables are not bold since the transformation corresponds to a single vortex-ring vertex. Positions and velocities of the collocation points are obtained through 
linear interpolation. The corresponding linear relations are given by

$$
\begin{aligned}
\left(\Delta \zeta_{b}\right)_{a}= & \Delta R_{a}-C^{a B} \widetilde{\xi}_{B} T \Delta \Psi, \\
\left(\Delta \dot{\zeta}_{b}\right)_{a}= & \widetilde{\omega}_{a}^{o} \Delta R_{a}+C^{a B}\left[\left(\widetilde{\xi}_{B} \Omega_{B}\right)-\widetilde{\xi}_{B} A_{1}-\widetilde{\xi}_{B} \widetilde{C^{B a}} \omega_{a}^{o} T\right] T \Delta \Psi \\
& +\Delta \dot{R}_{a}-C^{a B} \widetilde{\xi}_{B} T \Delta \dot{\Psi}+\Delta v_{a}-\left(\widetilde{R}_{a}^{o}+C^{a B} \widetilde{\xi}_{B} C^{B a}\right) \Delta \omega_{a},
\end{aligned}
$$

where $C^{a B}, T, \Omega_{B}$, and $A_{1}$ are evaluated at reference conditions, and the expression for the latter can be found in Ref. [176].

Second, the inviscid aerodynamic forces computed in Eqs. (13) are converted into forces and moments acting upon the beam nodes. They are expressed in the local aerodynamic frame, $A$, defined by the instantaneous non-circulatory velocity, and thus need to be transformed to the body-fixed $a$ frame, in order to be consistent with the flexible-beam equations, Eqs. (24). Finally, they are lumped into the nodes of the deformed beam, splitting them between adjacent ones. Integration of these nodal values yields the resultant forces and moments on the body-fixed FoR. These operations can be summarised as

$\boldsymbol{Q}_{a}^{S}=\left\{\begin{array}{c}\hat{\boldsymbol{F}}_{a} \\ \hat{\boldsymbol{M}}_{a}\end{array}\right\}=\chi_{v r \rightarrow n o} \bar{C}^{a A} \boldsymbol{F}_{A}$ and $Q_{a}^{R}=\chi_{n o \rightarrow b f} \boldsymbol{Q}_{a}^{S}$,

where $\bar{C}^{a A}\left(\zeta_{b}, \dot{\zeta}_{b}\right)$ is a block diagonal matrix, being each block given by the corresponding coordinate transformation matrix from the local aerodynamic to the body-fixed frame, $C^{A A}$. In turn, $\chi_{v r \rightarrow n o}=$ $\chi_{v r \rightarrow n o}\left(\boldsymbol{R}_{a}, \boldsymbol{\Psi}, \zeta_{b}\right)$ is a very sparse matrix that lumps the forces acting on the aerodynamic lattice, expressed in $a$, into forces and moments applied on the beam nodes, and $\chi_{n o \rightarrow b f}=\chi_{n o \rightarrow b f}\left(\boldsymbol{R}_{a}, \boldsymbol{\Psi}\right)$ integrates the nodal forces and moments to give the resultant loads at the bodyfixed frame.

In the linear case, it is assumed that matrices $\chi_{v r \rightarrow n o}$ and $\chi_{n o \rightarrow b f}$ remain constant, which is consistent with the frozen aerodynamic geometry assumption. However, as $\bar{C}^{a A}$ depends on grid velocities, this needs to be accounted for, leading to a linear mapping of the form

$\Delta \boldsymbol{Q}_{a}^{S}=\chi_{v r \rightarrow n o}^{o}\left[\left(\bar{C}^{a A}\right)^{o} \Delta \boldsymbol{F}_{A}+\left(\frac{\partial \bar{C}^{a A}}{\partial \dot{\zeta}_{b}}\right)_{o} \boldsymbol{F}_{A}^{o} \Delta \dot{\zeta}_{b}\right]$,

$\Delta Q_{a}^{R}=\chi_{n o \rightarrow b f}^{o} \Delta \boldsymbol{Q}_{a}^{S}$,

where $\Delta \boldsymbol{F}_{A}$ is given by Eqs. (19) and (20), $\Delta \dot{\boldsymbol{\zeta}}_{b}$ is obtained from Eq. (28), and the expressions to compute the tensor $\partial \bar{C}^{a A} / \partial \dot{\zeta}_{b}$ can be found in Ref. [168].

\subsection{Nonlinear time marching}

The aerodynamic and flexible-body equations have been outlined as independent modules, and their interdependency has been formulated as given by certain inputs, $\boldsymbol{u}$, and outputs, $\boldsymbol{y}$. For fully nonlinear time-domain simulations, the second-order continuous-time flexible-body equations are discretised in time and coupled with the discrete-time UVLM formulation.

The typical approach to march in time the flexible-body equations is the Newmark-beta method [4,176], which allows artificial damping to be introduced into the system, and is unconditionally stable for the right selection of parameters. Temporal discretisation of the beam equations enables the integrated model to be cast into the following compact notation:

$f_{A}\left(\boldsymbol{x}_{A}^{n+1}, \boldsymbol{u}_{A}^{n+1}\right)=g_{A}\left(\boldsymbol{x}_{A}^{n}, \boldsymbol{u}_{A}^{n}\right)$,

$f_{F B}\left(\boldsymbol{x}_{F B}^{n+1}, \boldsymbol{u}_{F B}^{n+1}\right)=g_{F B}\left(\boldsymbol{x}_{F B}^{n}, \boldsymbol{u}_{F B}^{n}\right)$,

where the state vectors are given in Eqs. (15) and (26), and the input vectors $\boldsymbol{u}_{A}$ and $\boldsymbol{u}_{F B}$ contain the fluid/structure mappings plus any other inputs to the system, such as gusts and controls. In particular, for the open-loop problem with no external disturbances (homogeneous problem), the inputs to the aerodynamic module only depend on the flexible-body outputs and vice versa, and the mapping relationships are expressed as

$\boldsymbol{u}_{A}^{n+\epsilon}=h_{A-F B}\left(\boldsymbol{y}_{F B}^{n+\epsilon}\right)$,

$\boldsymbol{u}_{F B}^{n+\epsilon}=h_{F B-A}\left(\boldsymbol{y}_{A}^{n+\epsilon}\right)$,

where the aerodynamic outputs, $\boldsymbol{y}_{A}=\boldsymbol{y}_{A}\left(\boldsymbol{x}_{A}, \boldsymbol{u}_{A}\right)$ are the aerodynamic loads given by Eqs. (13), and the flexible-body outputs and states coincide, such that $\boldsymbol{y}_{F B}=\boldsymbol{x}_{F B}$.

The time-domain solution of the equations of motion is a partitioned time-marching scheme. Strongly and weakly coupled solutions can be implemented, depending on whether a subiteration routine to converge aerodynamics and beam dynamics is included. The strong coupling includes subiterations, and might offer higher accuracy and better numerical stability properties. However, if a first-order-in-time explicit Euler method is used to convect the force-free wake, Eq. (10), the implementation of subiterations does not provide an advantage. The weakly coupled approach is found to work well with the appropriate selection of simulation parameters, and leads to a smaller computational burden.

The fully nonlinear time-marching solution described here accounts for large geometry changes, both in the structure and in the 3D aerodynamics, by updating the relevant inertia, gyroscopic and stiffness terms, and by enforcing the boundary conditions at the instantaneous deformed shape. In addition, the model includes an inviscid representation of the free aircraft-wake. This methodology, though necessary for very flexible structures, is computationally intensive compared to other potential-flow solvers, and does not lend itself to an accessible formulation for flight control system design or stability analysis. To that goal, the linearised version of the coupled equations is presented next.

\subsection{Monolithic integration of linearised equations}

Using the standard Newmark- $\beta$ method for the temporal discretisation, the linearised flexible-body equations, Eq. (25), are written as

$E_{F B}\left\{\begin{array}{l}\Delta \boldsymbol{x}_{S} \\ \Delta \boldsymbol{x}_{R}\end{array}\right\}^{n+1}+F_{F B}\left\{\begin{array}{l}\Delta \boldsymbol{u}_{S} \\ \Delta \boldsymbol{u}_{R}\end{array}\right\}^{n+1}=A_{F B}\left\{\begin{array}{l}\Delta \boldsymbol{x}_{S} \\ \Delta \boldsymbol{x}_{R}\end{array}\right\}^{n}+B_{F B}\left\{\begin{array}{l}\Delta \boldsymbol{u}_{S} \\ \Delta \boldsymbol{u}_{R}\end{array}\right\}^{n}$,

$\left\{\begin{array}{l}\Delta \boldsymbol{y}_{S} \\ \Delta \boldsymbol{y}_{R}\end{array}\right\}^{n}=\left\{\begin{array}{l}\Delta \boldsymbol{x}_{S} \\ \Delta \boldsymbol{x}_{R}\end{array}\right\}^{n}$.

Note that the output equation in the coupling with the UVLM is the full deformed state. In turn, the linear mapping relations given in Section 5.1 can be expressed as

$\Delta \boldsymbol{u}_{A}^{n}=p_{A S}^{n} P_{A S} \Delta \boldsymbol{y}_{S}^{n}+p_{A R}^{n} P_{A R} \Delta \boldsymbol{y}_{R}^{n}$,

$\Delta \boldsymbol{u}_{S}^{n}=p_{S A}^{n} P_{S A} \Delta \boldsymbol{y}_{A}^{n}$, and $\Delta \boldsymbol{u}_{R}^{n}=p_{R A}^{n} P_{R A} \Delta \boldsymbol{y}_{A}^{n}$,

where capital $P$ matrices represent the actual mapping and lowercase $p^{n}$ scalar values depend on the tuning parameters of the Newmark- $\beta$ and the time step.

As a result, assembling together the linearised UVLM, Eqs. (21), with the linearised flexible-body equations, Eqs. (33), and using the linear interface between both models, Eqs. (34), the resulting system can be cast into a monolithic discrete-time state-space formulation, which for the homogeneous problem has the form

$E_{\text {sys }} \Delta \boldsymbol{x}^{n+1}=A_{\text {sys }} \Delta \boldsymbol{x}^{n}$,

where the state vector that completely determines the linear system is

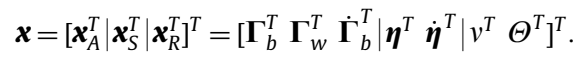

The coupling is monolithic in this case since the interface between aerodynamics and flexible body-dynamics is determined 
analytically. The linearisation might take place about a highly deformed configuration, and although the wake is frozen, interference-induced unsteady downwash effects are modelled.

From Eq. (35) a discrete-time generalised eigenvalue problem can be derived, which can be solved directly, without precomputing aerodynamic forces in the frequency domain or projecting on the structural modes, yielding a very powerful formulation for stability boundary prediction, as all derivatives have been obtained analytically.

The entries to matrices $E_{s y s}$ and $A_{s y s}$ depend on the equilibrium conditions. For the system to be stable, $\left|z_{i}\right| \leq 1, \forall i$, where $\left|z_{i}\right|$ represents the magnitude of the $i$ th discrete-time eigenvalue, and equality corresponds to the neutral stability boundary. Alternatively, the discrete-time eigenvalues can be transformed to the more familiar continuous-time counterparts $\lambda_{i}$, given by $z_{i}=e^{\lambda_{i} \Delta t}$. In this case, a positive real part of any of the $\lambda_{i}$-s will imply instability. Throughout this paper, unless otherwise stated, continuous-time eigenvalues will be presented for an easier interpretation.

In addition, as Eq. (35) represents a discrete-time state-space problem, it allows any type of analysis that is possible on a statespace system, such as time marching, reduced order modelling, and appending gusts and controls. For instance, in order to look at the closed-loop problem in which a controller is introduced for gust alleviation, the homogeneous problem shown in Eq. (35) is simply expanded as

$E_{s y s} \Delta \boldsymbol{x}^{n+1}=A_{s y s} \Delta \boldsymbol{x}^{n}+B_{d} \Delta \boldsymbol{u}_{d}^{n}+B_{c} \Delta \boldsymbol{u}_{c}^{n}$,

where $\boldsymbol{u}_{d}$ represents exogenous atmospheric disturbances and $\boldsymbol{u}_{c}$ control inputs (e.g., deflections of the aerodynamic surfaces). To conclude, note that a geometrically nonlinear beam model was used to introduce nonlinear static equilibrium conditions, but the analysis could be also based on the linear normal modes of the structure.

\section{Numerical examples}

By integrating the unsteady aerodynamics with the flexiblebody dynamics equations, the full aeroelastic and flight dynamics characterisation of the vehicle is completed. Other external forces, in particular, gravity forces, can also be included into the equations of motion. Different solution approaches can then be defined, including: tightly coupled static aeroelasticity, trim, asymptotic and Lyapunov stability, and open- and closed-loop dynamics simulations. Those should be carried out using linear or nonlinear solutions, when appropriate.

The results in this section mainly correspond to an implementation of such methodology carried out by the authors. A framework for simulation of high aspect ratio planes (SHARP) has been developed, built on a modular architecture in MATLAB, but with low-level libraries in Fortran. SHARP allows running independently the flexible-body dynamics and aerodynamics solvers, as well as the coupled system. Extensive verification of the tools has been carried out and these results, among others, have been reported in previous publications [17,18,185-187].

The main focus in this section is to illustrate the capabilities of the UVLM on the seamless integration of aero-, structural-, and rigid-body dynamics offered by the monolithic discrete-time statespace formulation. First of all, T-tail flutter results are presented in order to illustrate how the methodology naturally captures in-plane motions (Section 6.1). Next, linear stability analyses are performed for a representative HALE UAV model, first for the clamped flexible wing (Section 6.2), and then for the free-flying aircraft (Section 6.3). Parametric studies are carried out to determine the impact of velocity, payload location, and stiffness, while exemplifying the robustness of the method. Finally, the response of this aircraft to discrete gusts is assessed (Section 6.4), comparing linear and nonlinear solutions, and evaluating the influence of the force-free wake.

\subsection{T-tail flutter}

The aeroelastic stability of T-empennages has been subject to attention since the 1950s. As often happens, the problem was drawn to public attention after an unfortunate accident, in this case, that of the Handley Page Victor bomber in 1954 [188,189]. The key advantage offered by T-tails is that the horizontal tail plane (HTP) is clear of the wake shed by the main wing of the aircraft (particularly in high-wing configurations), consequently increasing its efficiency, reducing its size and averting buffeting [190]. In addition, it allows rear-mounted engines and it facilitates loading and unloading in military transport aircraft. The main pitfalls are the requirement of a stiffer vertical tail plane (VTP) in order to withstand the HTP weight, and the increased likelihood of deep stall. T-tails are also hampered by detrimental aeroelastic couplings that may arise due to the interplay of the bending/torsion modes of the VTP, and the aerodynamic interference with the horizontal stabiliser. The motions of the vertical fin introduce in-plane motions of the HTP, which are responsible for the creation of additional unsteady aerodynamic loads. The reader is referred to Ref. [191] for an introduction into the T-tail literature, and for a description on how to tackle T-tail flutter, including transonic effects.

The flutter of these tails is highly dependent on the steady lift of the HTP [192,193]. It also depends on its dihedral, and thus on the analogous effect induced by static deformations [194]. This highlights the importance of performing a linearisation of the equations for flutter computation based on the actual deformed geometry at the corresponding flight conditions.

In its standard formulation, the DLM includes neither steady loading nor in-plane motions, and is therefore not suitable for the prediction of flutter onset in these cases. It is however possible to incorporate these effects and accommodate the method for T-tail stability analysis [141,142]. In contrast, no modification is needed on the procedure based on the UVLM, which is able to capture all relevant kinematics and accounts for static aeroelastic effects. The methodology for stability evaluation is exercised next to illustrate this.

An empennage that consists of a vertical fin and an horizontal tail-plane is considered-the fuselage is not modelled. The lifting surfaces are thin, flat plates, with neither sweep nor dihedral. The assembly is clamped at the root of the vertical fin, and the joint between HTP and VTP is rigid. The main geometrical and structural properties of the test case are given in Table 1.

The two dominant modes that govern the stability of the tail are the torsion of the fin, which also introduces lead-lag motion of the HTP (Mode 1) and the bending of the fin (Mode 2). For this

Table 1

T-tail properties.

$\begin{array}{ll}\text { Chord of vertical fin } & 2 \mathrm{~m} \\ \text { Span of vertical fin } & 6 \mathrm{~m} \\ \text { Chord of HTP } & 2 \mathrm{~m} \\ \text { Semi-span of HTP } & 4 \mathrm{~m} \\ \text { Elastic axis (from l.e.) } & 25 \% \text { chord } \\ \text { Centre of gravity (from l.e.) } & 35 \% \mathrm{chord} \\ \text { Mass per unit length } & 35 \mathrm{~kg} / \mathrm{m} \\ \text { Sectional moment of inertia (around e.a.) } & 8 \mathrm{~kg} \mathrm{~m} \\ \text { Torsional stiffness } & 10^{6} \mathrm{~N} \mathrm{~m}^{2} \\ \text { Bending stiffness } & 10^{7} \mathrm{~N} \mathrm{~m}^{2} \\ \text { In-plane bending stiffness } & 10^{9} \mathrm{~N} \mathrm{~m}^{2}\end{array}$




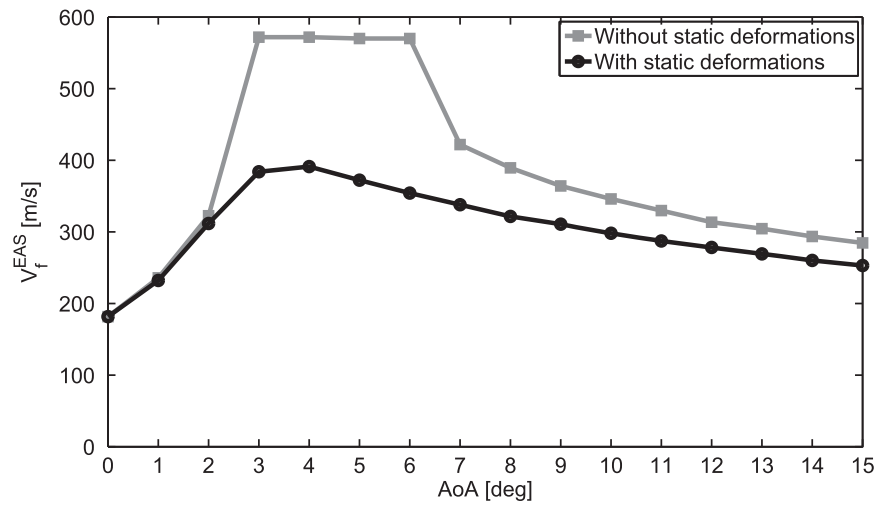

Fig. 4. Flutter speed of the T-tail as a function of angle of attack. Note the divergence speed at $V_{\infty}^{E A S}=570 \mathrm{~m} / \mathrm{s}$.

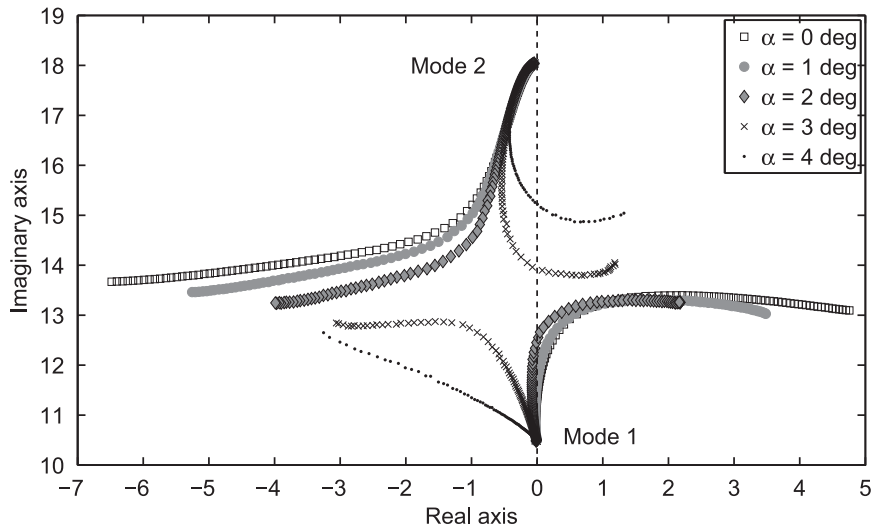

Fig. 5. Root loci for the T-tail with static deformations. $V_{\infty}^{E A S} \in[1,460] \mathrm{m} / \mathrm{s}$, $\Delta V_{\infty}^{E A S}=3.6 \mathrm{~m} / \mathrm{s}$.

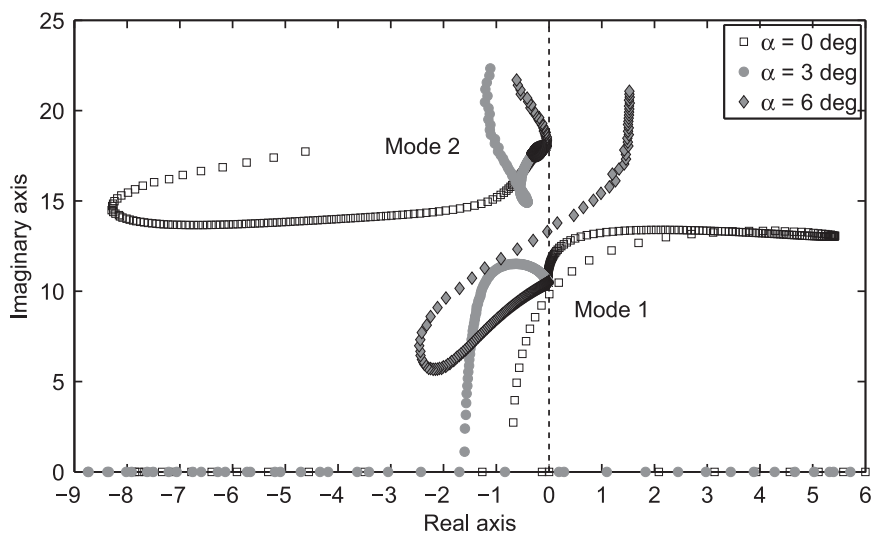

Fig. 6. Root loci for the T-tail without static deformations. $V_{\infty}^{E A S} \in[1,720] \mathrm{m} / \mathrm{s}$, $\Delta V_{\infty}^{E A S}=3.6 \mathrm{~m} / \mathrm{s}$

configuration, the in-vacuo frequencies of these modes are $\omega_{0}^{1}=10.5 \mathrm{rad} / \mathrm{s}$ and $\omega_{0}^{2}=18.0 \mathrm{rad} / \mathrm{s}$, respectively.

The flutter speed of the tail is computed for varying angles of attack (AoA), with and without static deformations. Results are shown in Fig. 4. The values of the flutter speed are presented as equivalent air speed (EAS), i.e, for a density of $\rho_{\infty}=1.225 \mathrm{~kg} / \mathrm{m}^{3}$. It can be observed that even though the static deformations are small, their influence on stability is significant, and throughout the range of angles, non-conservative.

When static deformations are included, the stability curve exhibits a maximum at an angle around $3^{\circ}$, and this is caused by a change in the fluttering mode. For smaller angles, the stability
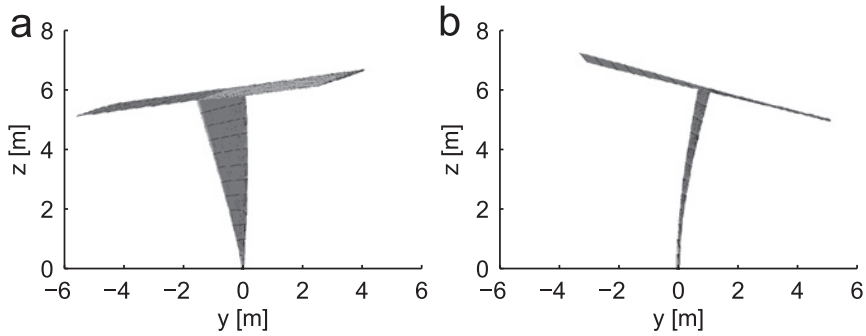

Fig. 7. T-tail modes with static deformations for $A o A=0^{\circ}$ and $V_{f}^{E A S}=182 \mathrm{~m} / \mathrm{s}$ (a) Mode 1 and (b) Mode 2.
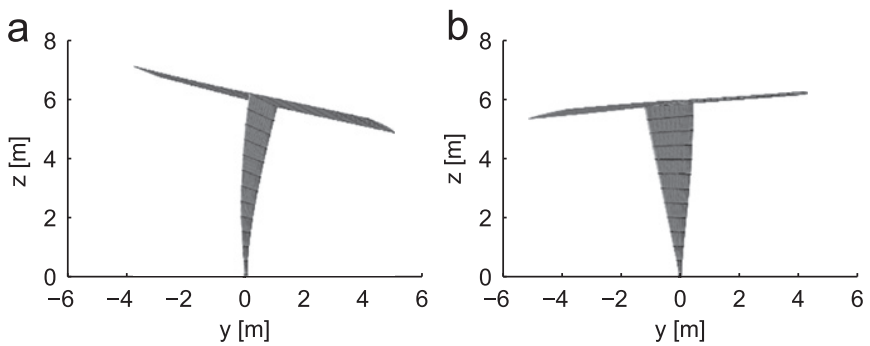

Fig. 8. T-tail modes with static deformations for $A o A=10^{\circ}$ and $V_{f}^{E A S}=298 \mathrm{~m} / \mathrm{s}$ : (a) Mode 1 and (b) Mode 2.

envelope grows with AoA, and it is Mode 1 which becomes unstable. For higher angles, it is Mode 2 which flutters, and the critical speed decreases with incidence. The stability of the tail without static deformations, though not correct, presents an even richer behaviour. Obviously, in the absence of aerodynamic loads, the flutter speed for zero angle of attack exactly matches the value with static deformations. The curves are very close for angles below $3^{\circ}$, but they then depart. The plateau found for the case when deformations are ignored corresponds to the divergence speed, which is reached before the flutter onset for $3 \leq \mathrm{AoA}<6$. Beyond this angle, Mode 2 is responsible for dynamic instability.

This can be more clearly visualised in the root loci. Fig. 5 displays the stability plot when static deformations are accounted for. Several angles of incidence are included to expose the transition between modes. In these diagrams, a positive real part indicates instability, and only the upper halves of the root loci are included, since they are symmetric due to the complex conjugate nature of the eigenvalues. As it can be seen, the change occurs when the angle increases from $2^{\circ}$ to $3^{\circ}$, and the mode that flutters switches from Mode 1 to Mode 2.

The root loci for the case without static deformations are displayed in Fig. 6. More data points are needed here until instability is reached. A zero angle of incidence shows a very interesting feature. Mode 1 becomes unstable early on, but continuation on the stability plot evidences that it returns back to stability as the speed grows, only to become unstable again as a zero-frequency pole. For $\mathrm{AoA}=3^{\circ}$, Mode 1 is stable for a wide range of velocities, until it hits the abscissa axis, becomes non-oscillatory, and crosses the stability boundary, which represents divergence. In turn, for $\mathrm{Ao} A=6^{\circ}$, Mode 1 remains oscillatory throughout, and eventually flutters. For higher angles of attack, it is Mode 2 the one that becomes unstable as for the situation when static deformations are included.

While at small angle of attacks there is clear distinction between the torsion and bending modes of the vertical fin, the division is blurred as the angle increases and aerodynamic loading, both steady and unsteady, gains prominence. Figs. 7 and 8 depict the two dominant eigenmodes at flutter onset for 

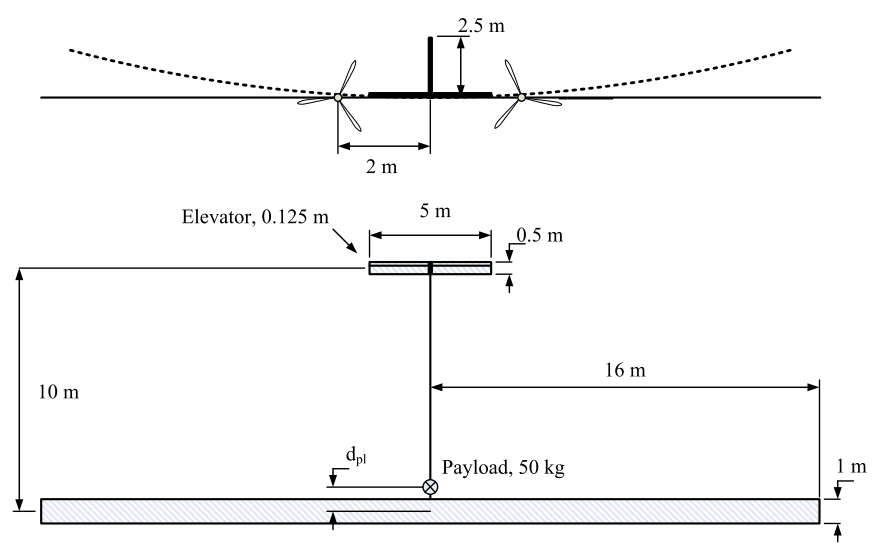

Fig. 9. HALE model aircraft geometry. Large-aspect-ratio straight wing, rigid fuselage and tail, and propellers (not to scale). Front and top views showing typical wing deformation.

Table 2

HALE model aircraft properties.

\begin{tabular}{lll}
\hline Property & Main wing & Tail \\
\hline Chord & $1 \mathrm{~m}$ & $0.5 \mathrm{~m}$ \\
Semi-span & $16 \mathrm{~m}$ & $2.5 \mathrm{~m}$ \\
Elastic axis (from l.e.) & $50 \%$ chord & $50 \%$ chord \\
Centre of gravity (from l.e.) & $50 \% \mathrm{chord}$ & $50 \% \mathrm{chord}$ \\
Mass per unit length & $0.75 \mathrm{~kg} / \mathrm{m}$ & $0.08 \mathrm{~kg} / \mathrm{m}$ \\
Moment of inertia (around e.a.) & $0.1 \mathrm{~kg} \cdot \mathrm{m}$ & $0.01 \mathrm{~kg} \mathrm{~m}$ \\
Torsional stiffness & $\sigma \times 10^{4} \mathrm{~N} \mathrm{~m}^{2}$ & $\infty$ \\
Bending stiffness & $2 \sigma \times 10^{4} \mathrm{~N} \mathrm{~m}^{2}$ & $\infty$ \\
In-plane bending stiffness & $4 \sigma \times 10^{6} \mathrm{~N} \mathrm{~m}^{2}$ & $\infty$ \\
\hline
\end{tabular}

$\mathrm{Ao} A=0^{\circ}$ and $\mathrm{Ao} A=10^{\circ}$, respectively, including static deformations. These shapes are obtained by plotting the relevant normalised eigenvectors, directly computed from the eigenvalue analysis to evaluate stability. Whereas the eigenshapes at zero incidence are very similar to those in vacuo, fin bending and torsion appear coupled for larger angles, and help to explain the transition between fluttering modes.

The above example highlights the potential of the UVLM to capture in-plane motions, and to incorporate static deformations, both of which prove to be pivotal in T-tail flutter, and to integrate static and dynamic stability in the same analysis.

\subsection{Stability of a flexible wing}

Flutter results for a flexible wing are presented in this section. For that purpose, a numerical model of a HALE UAV has been defined, with main features given in Fig. 9 and Table 2, based on the one proposed by Patil and co-workers [10]. The vehicle consists of a large aspect-ratio flexible wing, a rigid fuselage and a rigid tail comprising a $25 \%$ chord elevator. Both the wings and the tail surfaces are modelled as uncambered thin flat plates with aerodynamic effects, and the fuselage is a non-lifting body. The aircraft carries a payload of $50 \mathrm{~kg}$ in the fuselage (the only non-structural mass of the problem, modelled as a point load), located at a distance $d_{p l}$ from the elastic axis of the main wing, and is powered by two propellers, which are modelled as point forces rigidly linked to the wing. The mass per unit length of the fuselage is the same as that of the tail members, and thus the total mass of this aircraft, including payload and structural mass, is $75.4 \mathrm{~kg}$. As observed in Table 2, the stiffness properties of the main wing will be used as a parameter for subsequent results, varying the parameter $\sigma$-the flexibility of the wing increases as $\sigma$
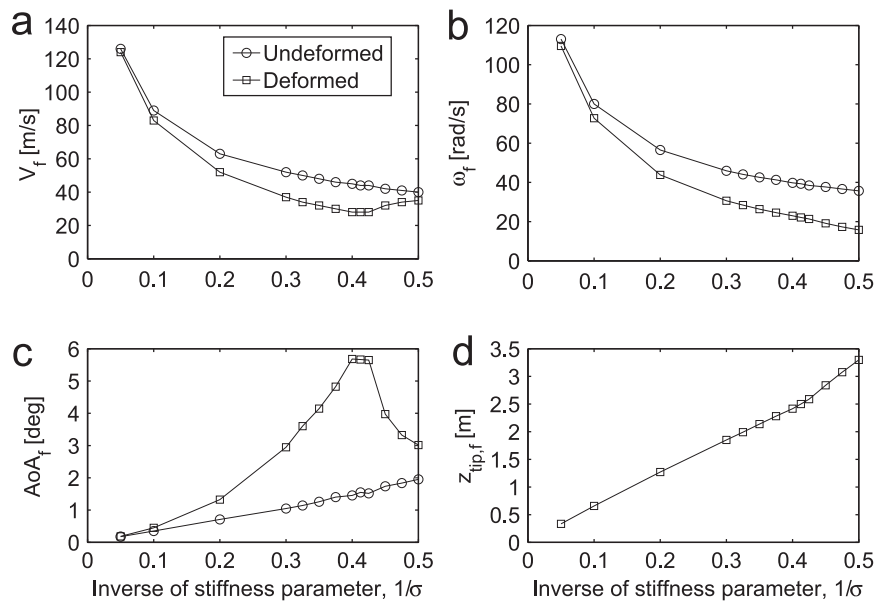

Fig. 10. Aeroelastic stability of a flexible wing. Matched flutter conditions as a function of wing flexibility, versus linearisation about the undeformed configuration: (a) flutter speed, (b) flutter frequency, (c) angle of attack, and (d) static wingtip deflection.

decreases. It is assumed that the aircraft flies at an altitude of $20 \mathrm{~km}$, where the density is $\rho_{\infty}=0.0899 \mathrm{~kg} / \mathrm{m}^{3}$.

In this section the aircraft is assumed to be clamped (rigidbody degrees-of-freedom are not included) and the payload is on the elastic axis, $d_{p l}=0 \mathrm{~m}$. The full aircraft description is required for trimming the aircraft, i.e., for steady level flight. However, as the tail and the fuselage are rigid, the aeroelastic stability is solely dependent on the main wing-note that the tail would affect stability if rigid-body degrees of freedom were included, as it will be seen in Section 6.3. The trimming is achieved through three inputs, namely angle of attack, AoA, elevator deflection, $\delta$, and thrust per propeller, $T$.

The solution process is as follows: for a given free-stream velocity, the trim conditions are computed and a linear stability analysis is carried out about the deformed configuration that corresponds to these trim inputs. This is accomplished by solving directly the generalised eigenvalue problem derived from Eq. (35). If the wing is stable, the flight speed is increased, the aircraft trimmed and the stability analysis performed again. This is repeated until the flutter onset is found, and is therefore a matched solution-this is the notation typically used with respect to the Mach number.

Fig. 10 depicts the results of the matched flutter calculations for varying stiffness properties of the main wing. Results are plotted against the inverse of the stiffness parameter so that the wing flexibility increases as the abscissa grows. In order to evaluate the effect of the static deformations, the linearisation is also performed about the undeformed wing for the same trim conditions. Alongside the flutter speed and frequency, the angle of attack that trims the aircraft at the flutter speed, and the wing-tip deflection for this incidence are also presented. As one would expect, linearising around the undeformed configuration leads to a significant overestimation of the flutter onset on a very flexible wing, which may lead to catastrophic consequences. In fact, even for a relatively stiff wing, $1 / \sigma=0.1$, which corresponds to a tip deflection of the order of $4 \%$ of the wing semi-span, the discrepancy is already noticeable. As the flexibility increases, this difference grows dramatically (61\% error at $1 / \sigma=0.4$ ), until a minimum is found for the flutter speed of the deformed configuration.

For the stiffest wings, $1 / \sigma \leq 0.4$, the mode that becomes unstable is the coupling of the bending and torsion degrees of freedom of the wing. This is the case for the linearisation about both the undeformed and the deformed shapes. As flexibility increases, the mode that flutters for the undeformed case remains 

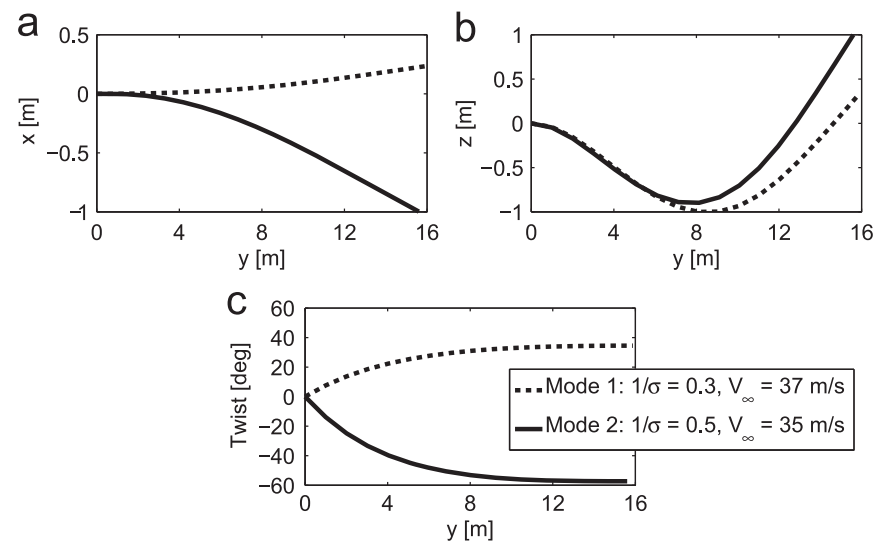

Fig. 11. Fluttering modes of the flexible wing: (a) in-plane displacements, (b) outof-plane displacements, and (c) twist of the reference line.

the same-obviously, the frequency is modified. In contrast, the flutter mechanism for the deformed wing as $1 / \sigma$ increases includes a strong chordwise bending (in-plane) component that couples with the torsion and spanwise bending degrees of freedom. This explains the growing differences in flutter frequency while the flutter speeds remain relatively close. The relevance of in-plane bending modes in aeroelastic analysis was highlighted, for instance, in Ref. [89].

Fig. 11 depicts these two modes when linearising about the deformed configuration. For that purpose, two different stiffnesses have been considered and the information obtained from the normalised eigenvectors at the respective flutter speeds are plotted. For $1 / \sigma=0.3$ the flutter onset occurs at $V_{\infty}=37 \mathrm{~m} / \mathrm{s}$, and what has been dubbed as Mode 1 is responsible for instability. In contrast, for $1 / \sigma=0.5$ the neutral speed corresponds to $V_{\infty}=35 \mathrm{~m} / \mathrm{s}$, and it is Mode 2 that flutters. While the spanwise bending profile is similar in both cases, chordwise deformations and torsion of the reference line present absolutely different characteristics. The significant in-plane deformations featured by Mode 2 can be clearly observed.

The change in flutter mode becomes obvious through the angle of attack at the matched flutter conditions - see Fig 10(c). The curve presents a maximum at the values of stiffness at which the change in behaviour occurs for the deformed shape. This result is entirely due to the emergence of a different flutter mechanism. Trim inputs evolve smoothly across the ranges of velocities and flexibilities considered, and there is no discontinuity in the derivative of the function. This is illustrated by the curves for the undeformed wing: as the unstable mode is the same for all values of $\sigma$, the required angle of attack increases as the flutter speed decreases so that the aircraft remains trimmed. The matched flutter procedure followed here permits the identification of this bifurcation in the stability characteristics of the wing.

The static wing-tip deflection increases linearly with wing flexibility, since the total wing lift remains roughly constant to balance the total weight. It is complicated to anticipate the required trim inputs for an aircraft with a very flexible wing. On the one hand, as the deflections grow, the angle of attack needs to increase in order to counteract the loss of vertical force caused by the tilting of the lift vector. On the other, the twist of the wing grows rapidly with wing flexibility and provides additional lift force. Furthermore, as the wing deforms the direction along which the thrust force acts changes significantly. Thus, the exact load distribution for equilibrium follows from the intricate interaction and balance of all these factors.

Finally, note that results beyond the values of flexibility considered are not presented because trimming the aircraft becomes

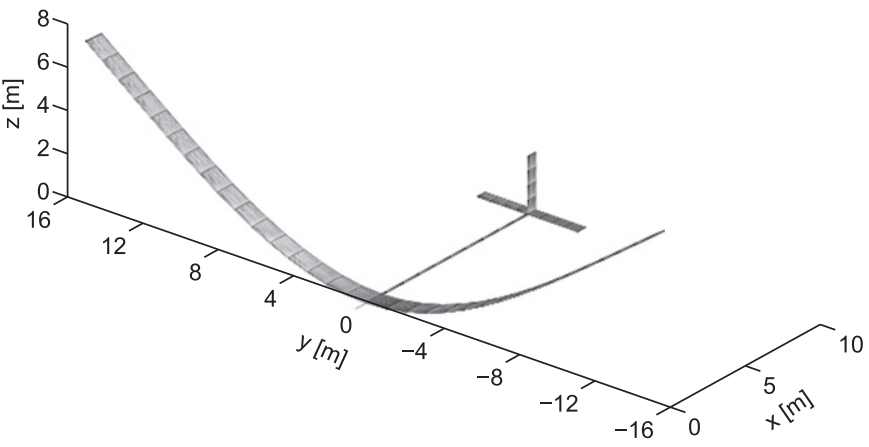

Fig. 12. Deformed configuration of the model aircraft at trim conditions for $\rho_{\infty}=0.0889 \mathrm{~kg} / \mathrm{m}^{3}, V_{\infty}=25 \mathrm{~m} / \mathrm{s}, \sigma=1$, and $d_{p l}=0 \mathrm{~m}$.

Table 3

Longitudinal continuous-time eigenvalues of the rigid aircraft.

\begin{tabular}{llll}
\hline Mode & $V_{\infty}=20 \mathrm{~m} / \mathrm{s}$ & $V_{\infty}=25 \mathrm{~m} / \mathrm{s}$ & $V_{\infty}=30 \mathrm{~m} / \mathrm{s}$ \\
\hline Phugoid (quasi-steady) & $-0.051 \pm 0.361 i$ & $-0.022 \pm 0.288 i$ & $-0.011 \pm 0.240 i$ \\
Phugoid (UVLM) & $-0.054 \pm 0.365 i$ & $-0.028 \pm 0.269 i$ & $-0.016 \pm 0.218 i$ \\
Real root (quasi-steady) & -3.21 & -4.08 & -4.93 \\
Real root (UVLM) & -1.96 & -2.71 & -3.44 \\
Real root (quasi-steady) & -9.27 & -11.60 & -13.91 \\
Real root (UVLM) & -10.61 & -13.14 & -17.18 \\
\hline
\end{tabular}

infeasible for near-flutter speeds. This in fact sets an upper boundary to the achievable flight velocities.

\subsection{Linear stability analysis of an aircraft with coupled flight dynamics and aeroelastic response}

As the flexibility of their primary structures increases, aircraft are prone to exhibit unconventional features, such as an overlap of structural and rigid-body degrees of freedom, that is, of its aeroelastic and flight dynamic characteristics. In order to exemplify this, the configuration introduced above (see Fig. 9 and Table 2) is considered again. Results are also obtained at the same flight altitude. Fig. 12 shows the deformed shape of the trimmed aircraft for the nominal conditions of $V_{\infty}=25 \mathrm{~m} / \mathrm{s}, \sigma=1$, and $d_{p l}=0 \mathrm{~m}$, which highlights the very large deformations that can appear on the main wing of this configuration. In the results in the figure, the vertical tip deflection reaches $44 \%$ of the semi-span, with an inward tip displacement of nearly $2 \mathrm{~m}$.

First of all, the impact of the flight speed on the longitudinal motions of the rigid aircraft is illustrated. For this case, based on a quasi-steady aerodynamics approximation, it is possible to obtain a system of equations for the four longitudinal rigid-body states [152, p. 175], which allows closed-form solution of the stability derivatives. The results of this simplified model are compared to those obtained with the UVLM, solving the eigenvalue problem derived from Eq. (35) for the free-flying rigid vehicle at the corresponding trim conditions. In this problem, the elastic states are neglected, and hence the corresponding rows and columns are removed from the discrete-time state-space formulation.

The rigid vehicle is trimmed at free-stream speeds of $V_{\infty}=20$, 25 , and $30 \mathrm{~m} / \mathrm{s}$, and the stability is evaluated for the corresponding conditions, both for the quasi-steady approximation and the UVLM. Table 3 summarises these results.

The solution of the eigenvalue problem gives as many eigenvalues as states has the system, and it includes lateral as well as longitudinal modes. For comparison purposes, only the four dominant longitudinal eigenvalues are included in Table 3 . The agreement between quasi-steady aerodynamics and the UVLM is 


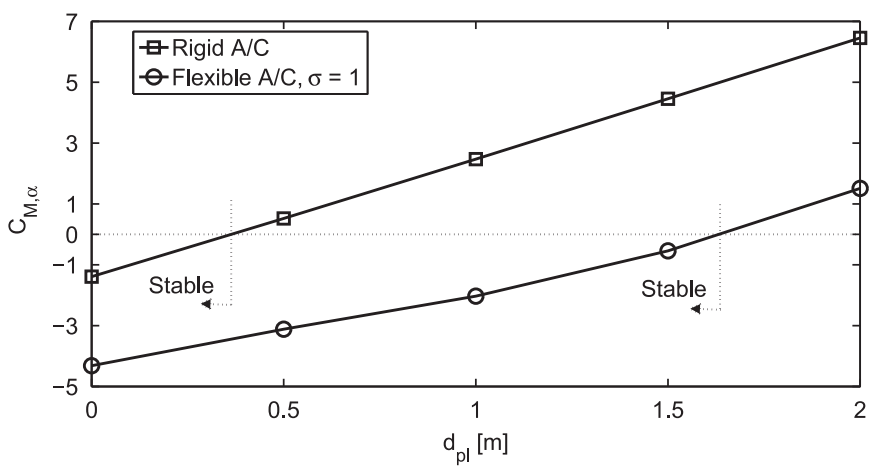

Fig. 13. Static stability of the rigid and flexible aircraft as a function of the location of the payload, $d_{p l}$, for $\rho_{\infty}=0.0889 \mathrm{~kg} / \mathrm{m}^{3}$, and $V_{\infty}=25 \mathrm{~m} / \mathrm{s}$.

Table 4

Trim characteristics: inputs and tip deflection.

\begin{tabular}{llccc}
\hline Case & Input & $V_{\infty}=20 \mathrm{~m} / \mathrm{s}$ & $V_{\infty}=25 \mathrm{~m} / \mathrm{s}$ & $V_{\infty}=30 \mathrm{~m} / \mathrm{s}$ \\
\hline \multirow{2}{*}{ Rigid } & Angle of attack (deg) & 13.2 & 8.2 & 5.6 \\
& Elevator (deg) & -7.4 & -4.4 & -2.9 \\
& Thrust (N) & 7.6 & 4.6 & 3.1 \\
\multirow{5}{*}{ Flexible } & Angle of attack (deg) & 16.0 & 8.7 & 5.3 \\
& Elevator (deg) & -23.8 & -9.5 & -4.4 \\
& Thrust (N) & 8.8 & 5.6 & 4.3 \\
& Tip displacement (m) & 6.6 & 7.1 & 7.7 \\
\hline
\end{tabular}

remarkable for the phugoid mode. This mode depends heavily on the variation of horizontal forces with flight speed, and so is governed by the induced drag. Even though the estimation of induced drag is different in both cases, it seems obvious that for this very high-aspect-ratio wing the effect is minor.

The second characteristic longitudinal mode, namely the shortperiod mode, does not appear in its conventional form here. Instead of a pair of complex conjugate oscillatory roots, two negative real eigenvalues are obtained. Even though the trends provided by the quasi-steady aerodynamics are consistent with those of the UVLM, the discrepancy here is bigger. This attests the errors incurred by the quasi-steady model. These non-oscillatory poles are strongly linked to the static margin of the aircraft. In fact, for a statically unstable vehicle, i.e., when the aerodynamic centre is ahead of the centre of gravity $\left(\partial C_{M} / \partial \alpha>0\right)$, one of the real roots would be positive. For instance, by displacing the payload along the fuselage towards the tail, the aircraft becomes pitch unstable. Fig. 13 illustrates this, plotting $C_{M, \alpha}$ for different payload locations, $d_{p l}$, both for the rigid and flexible vehicles. $C_{M, \alpha}$ is determined at the trim conditions for each payload position, and is computed via finite differences. The most notable feature of this figure is that the flexible case exhibits a larger range of $d_{p l}$ for static stability. Note that for higher values of $d_{p l}$ the trim conditions become unrealistic, as the required elevator deflection becomes excessive, and thus are not included.

Following with the assessment of flight-speed influence on stability, the flexible aircraft is considered next. In this case, the nominal value of the stiffness is taken, $\sigma=1$, and results are compared to the rigid aircraft. From now on, $d_{p l}=0 \mathrm{~m}$ to guarantee static stability. Table 4 lists the trim features for the rigid and flexible aircraft for the three free-stream velocities simulated, where the thrust given is for each propeller.

Fig. 14 presents the root loci as a function of flight speed, showing the dominant modes, Fig. 14(a), and magnifying the region closest to the origin, Fig. 14(b). Only the upper halves of root loci are included. The poles that correspond to the same modes have been linked to aid
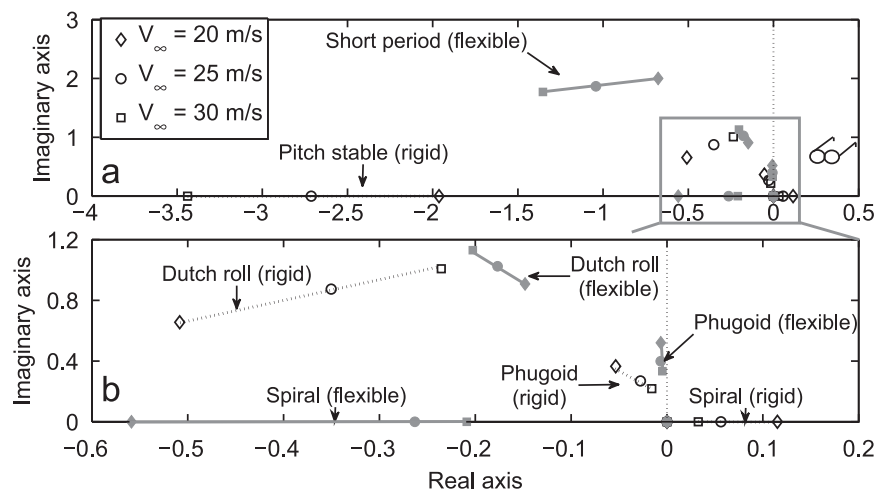

Fig. 14. Root loci of the eigenvalue analysis for the flexible and rigid aircraft, with the flight velocity as parameter: (a) dominant roots and (b) magnified section showing the roots nearest the origin.

interpretation. This continuation can be deduced by evaluating the shape of the corresponding eigenvectors.

One of the negative real roots introduced in Table 3 can be easily identified in Fig. 14(a), and it proves pitch stability. The root locus also reveals the short-period mode for the flexible aircraft, whereas the equivalent for the rigid case is beyond the range displayed. Note that many roots are left out of the plotted area, since they are either very high-frequency modes, or because they have very large negative real parts, and are therefore very stable. The latter includes roll subsidence and yaw stiffness, both zerofrequency stable roots, which correspond to self-correcting mechanisms that resist roll rate and sideslip, respectively.

Fig. 14(b) zooms in the section highlighted in Fig. 14(a) to study the eigenvalues in more detail. Regarding oscillatory modes, the typical longitudinal phugoid and lateral Dutch-roll can be identified for the rigid vehicle, and their counterparts for the flexible aircraft are also shown. ${ }^{2}$ The effect of wing flexibility is manifest. Although still stable, the damping of the flexible phugoid mode is about an order of magnitude smaller than its rigid equivalent. The stability margin of the rigid phugoid decreases as the flight speed increases, but the flexible mode displays a distinct behaviour, for which damping remains nearly constant. In both cases the frequency of the mode decreases with flight speed. The discordance in Dutch roll is even more radical. While the frequency grows with velocity in both cases, the trend in damping is opposite for the rigid and flexible vehicle.

The characteristic spiral mode can also be seen in Fig. 14(b). This is a non-oscillatory motion characterised by (zero-frequency) real roots. The mode is unstable for the rigid aircraft at the flight velocities considered, which is an expected outcome, since the wings do not have dihedral. In contrast, as the wings exhibit large deformations at the trim conditions (see Table 4), they provide resilience against the spiral mode, analogous to the dihedral effect, and the flexible aircraft is thus stable. Interestingly, the increase in speed leads to opposite tendencies in damping for rigid and flexible cases. Finally, note that as the yaw angle is included as a state of the system, a neutral mode appears at the origin in all cases, but is of no interest.

Next, the effect of the stiffness of the main wing is exemplified. In this case the flight speed is fixed at $V_{\infty}=25 \mathrm{~m} / \mathrm{s}$ and the stiffness of the main wing is used as parameter and varied from the nominal value, $\sigma=1$, to rigid, $\sigma=\infty$. From $\sigma=1$ to $\sigma=2.5$ the stiffness is varied in increments of $\Delta \sigma=0.1$, and from $\sigma=3$ to

\footnotetext{
${ }^{2}$ The first few modes of the flexible vehicle will be identified by the names on their rigid-aircraft counterparts, (e.g., phugoid, short period, etc.), although they also include vehicle deformations.
} 

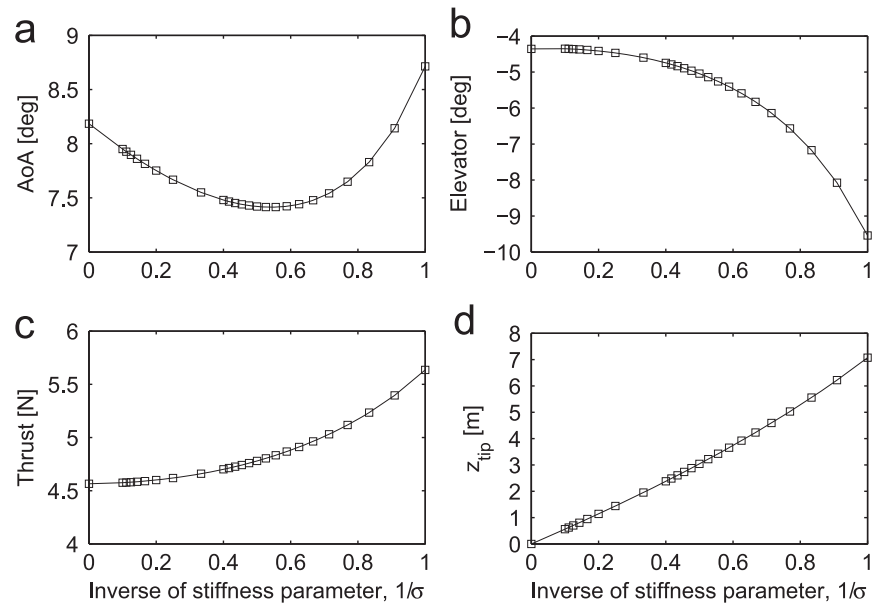

Fig. 15. Trim characteristics of the flexible aircraft at $V_{\infty}=25 \mathrm{~m} / \mathrm{s}$ as a function of wing flexibility: (a) angle of attack, (b) elevator deflection, (c) thrust, and (d) static wing-tip deflection.

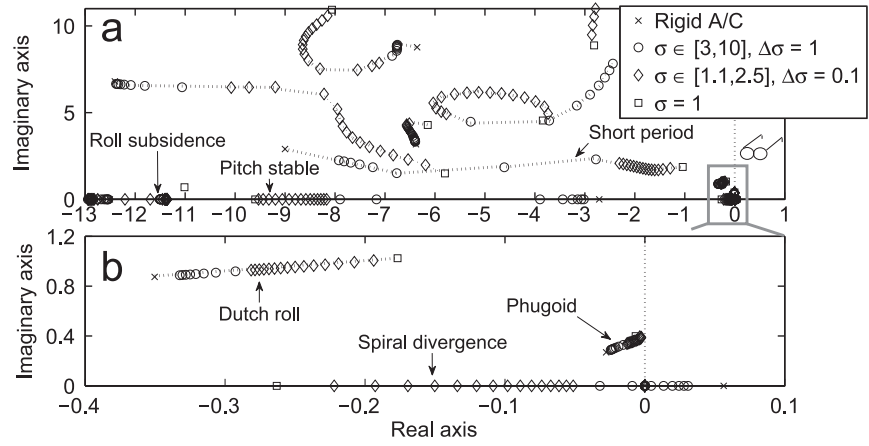

Fig. 16. Root loci of the eigenvalue analysis for the flexible and rigid aircraft at $V_{\infty}=25 \mathrm{~m} / \mathrm{s}$, with the stiffness of the main wing as parameter: (a) dominant roots, and (b) magnified section showing the roots nearest the origin.

$\sigma=10$, in increments of $\Delta \sigma=1.0$. First of all, the aircraft is trimmed in order to compute the deformed configuration about which the linear stability analysis will take place. Trim results are shown in Fig. 15, and it can be clearly seen from the tip displacements of the wings, that they may correspond to highly deformed wing shapes. Hence, the linear stability analysis is performed about a nonlinear static equilibrium configuration.

Fig. 16 presents the root loci as a function of $\sigma$, showing the dominant modes and zooming in the region of interest. As mentioned earlier, one of the flight-dynamics modes is the roll subsidence mode, associated to a resistance of the vehicle to roll. This mode is identified in Fig. 16(a), and it is characterised by large damping, making it very stable. An analogous behaviour to roll occurs in yaw. While this is not one of the classical modes obtained from the lateral stability quintic, it is mentioned in the literature as yaw stiffness [153], and it is also characterised by a real stable root. This mode is beyond the boundaries of this root locus, given by negative real poles with a larger damping than the roll subsidence mode. The pitch stability of the aircraft can also be attested from the figure. As the flexibility of the wing increases, the static margin becomes more prominent, which is consistent with the results presented in Fig. 13.

One of the modes that most conspicuously epitomises wing flexibility is the short period. As the stiffness of the wing is reduced, its damping rapidly decreases and approaches the neutral stability boundary. From the rigid case to the nominal stiffness of $\sigma=1$, the damping plummets by an order of magnitude. For the set of conditions studied, it remains stable, but the effect of static deformations and the coupling of the rigid-body mode with the wing elasticity is obvious. The short period has a higher frequency than the phugoid, and is likely to overlap with the structural frequencies of flexible wings, which are much smaller than those of very stiff aircraft. Several other modes are also shown in this diagram, and the significance of couplings between rigid-body and elastic modes is evident.

Fig. 16(b) focuses on the region closest to the origin. The trend observed before for the spiral divergence (Fig. 14) is even more clearly highlighted here. The mode is originally unstable for the rigid configuration, but the eigenvalue moves to the left of the root locus as the stiffness decreases. This illustrates the instrumental dihedral effect provided by the bending of the wing, playing a stabilising role, more significant as deformations grow. The classical Dutch roll is captured in this figure as well. Analogous to the short period, the damping falls with wing flexibility inducing a destabilising effect.

Finally, the phugoid appears as the most lightly damped oscillatory mode, and gets very close to the neutral boundary as the wing excursions grow. This flexible phugoid is characterised by a longitudinal constant angle-of-incidence motion coupled with the first bending mode of the wing. In contrast to the short period or the Dutch roll, nonetheless, the initial damping reduction with wing flexibility is eventually reversed, and the poles displace towards the left of the root locus (that is, an increase in wing flexibility beyond a certain value has a stabilising effect on the vehicle).

Some of the key modes are graphically illustrated next for the nominal conditions of $V_{\infty}=25 \mathrm{~m} / \mathrm{s}, \sigma=1$, and $d_{p l}=0 \mathrm{~m}$. The modes are obtained from the corresponding normalised eigenvectors. In order to ease visualisation, the structural states are multiplied by 10 (with respect to the rigid-body degrees of freedom). Different views of the motion are presented, and snapshots have been taken at different intervals during one full cycle (of the relevant mode) to highlight salient features in each of them. The modes are damped, but the damping is ignored when plotting them.

The flexible phugoid mode is displayed in Fig. 17. Fig. 17(a) presents a lateral view of the trajectory followed by the aircraft, which shows the pitching and plunging motions that counteract each other for a constant angle of incidence. The wing excursions around the static equilibrium shape can also be perceived. Fig. 17(c-f) highlights the deformations of the main wing. As the aircraft pitches up and climbs first, the wings undergo a downward flapping-like motion. As the vehicles pitches down and descends, the wing bends upwards, reaching the maximum tip deflections before the cycle is finished. The snapshots of the ascending and descending trajectories at the same altitude indicate the measure of the wing deflections. Fig. 17(b) summarises the above features. Note that the position of the aircraft is not part of the states of the system, but it is obtained by integrating body velocities.

As aforementioned, the coupling of elastic deformations with the short-period mode is more prominent than in the phugoid. Fig. 18 proves this. Again, the eigenvector is normalised and structural states magnified by a factor of 10 . Fig. 18(a), (b), and (c-f) shows lateral, perspective, and front views of the trajectory, respectively. Clearly, the deformations of the wing are much larger in this case. The higher frequency of the short period can be inferred from the distance travelled in one period, and while the amplitude of the pitching motion is comparable to that of the phugoid, the plunging amplitude is much smaller. This stability mode has also been referred to as body-freedom flutter in the literature [195].

Note that the phugoid in Fig. 17 is dominated by slow vertical motions of the aircraft, and the inertial coupling with the first bending mode of the wing yields in-phase elastic deformations (when the aircraft goes up, the wing goes down). The short-period 

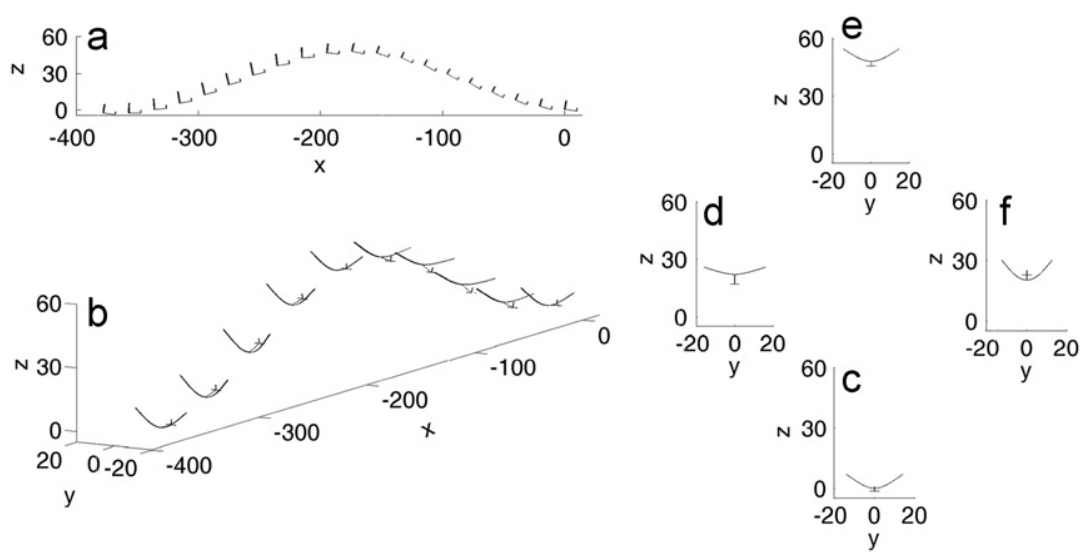

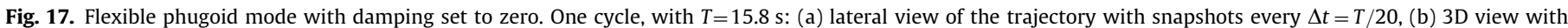
snapshots every $\Delta t=T / 10$, and (c-f) front views with snapshots every $\Delta t=T / 4$, clockwise. All dimensions $(x, y, z)$ in metres.
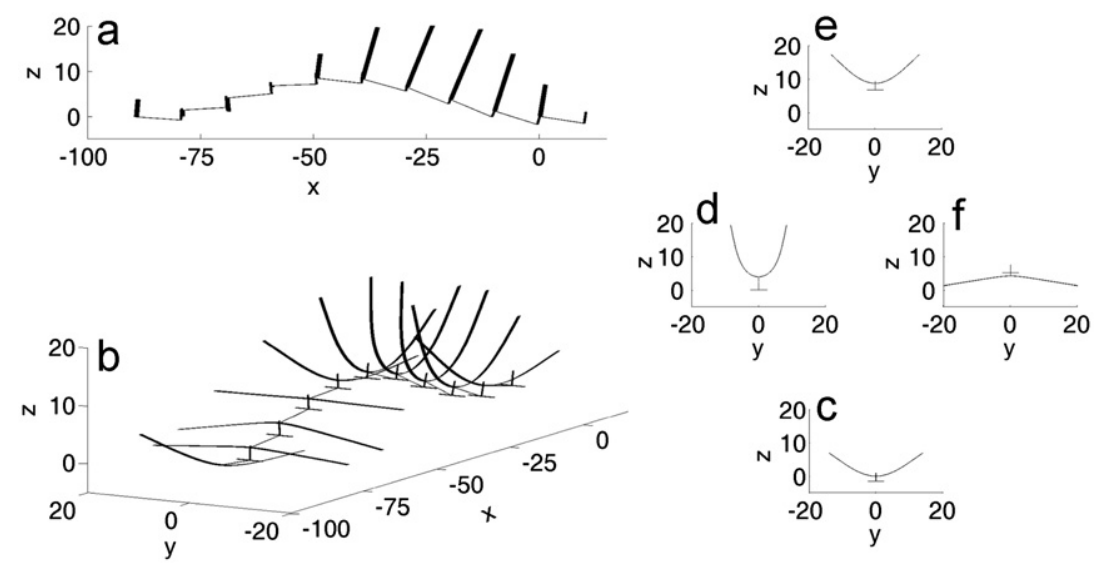

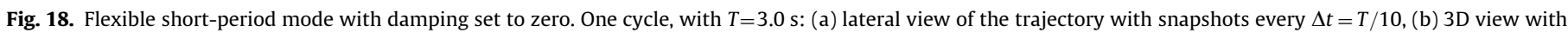
snapshots every $\Delta t=T / 10$, and (c-f) front views with snapshots every $\Delta t=T / 4$, clockwise. All dimensions $(x, y, z)$ in metres.

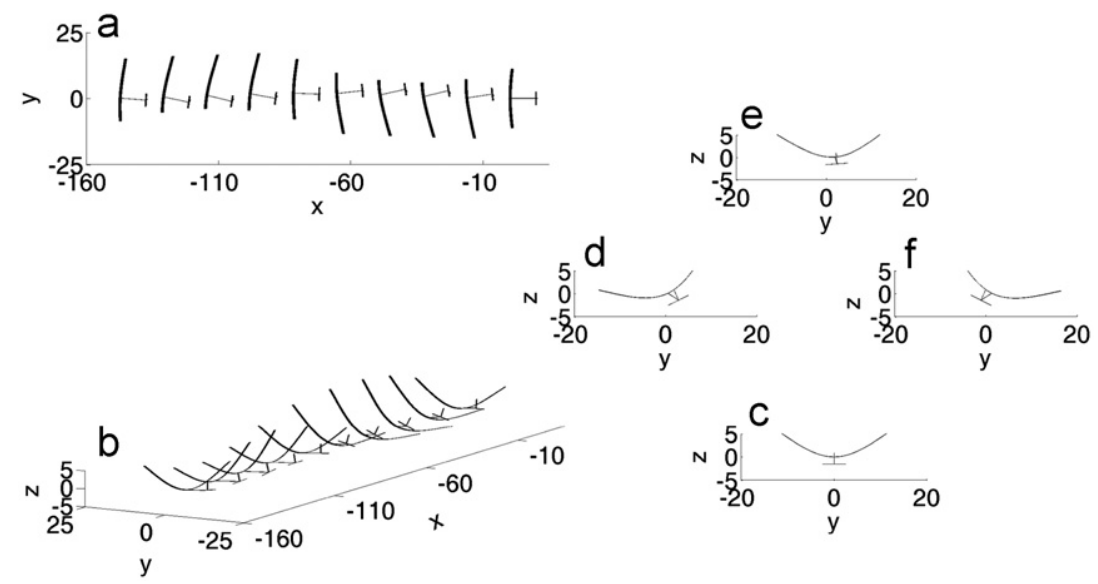

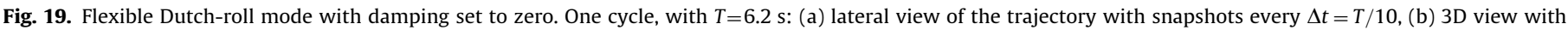
snapshots every $\Delta t=T / 10$, and (c-f) front views with snapshots every $\Delta t=T / 4$, clockwise. All dimensions $(x, y, z)$ in metres.

also involves a change on the incidence angle, and that makes the interaction with the elastic degrees of freedom more complex: there is the inertial coupling as in the phugoid mode, but a second aerodynamic coupling also appears between the changes in lift due to changes in induced angle of attack, and the deformations of the wing. As a result, the wing bending is out of phase with the vertical rigid-body motions. If the short period happens at highenough reduced frequencies, there would be also an effect of the aerodynamic lags on this coupling.

Finally, the Dutch roll is displayed as an example of a lateral mode in Fig. 19. Top, perspective and front views of the Dutch roll trajectory have been taken in Fig. 19(a), (b), and (c-f). It can be 
seen in this case that the amplitude of structural deformations is negligible against the rolling, yawing and plunging encompassed by the aircraft motion.

\subsection{Open-loop gust response of a UAV platform}

During their life-cycle aircraft experiences static, dynamic, and even impact loads. Loads due to ground and flight manoeuvres, atmospheric disturbances, or foreign-object strikes are critical for the successful design of the vehicle. The correct estimation of the loads the aircraft will be subject to is therefore essential, and depends on aerodynamics, structures, and weights. In particular, time-dependent forces that affect the dynamic aeroelastic behaviour of the aircraft might represent very demanding load cases, and must be studied as a transient-dynamics problem.

Some of the most critical dynamic-load conditions are those caused by encounters with gusts and atmospheric turbulence [60,196-198]. This is particularly critical in the case of very flexible UAVs, which still face a major structural integrity risk in adverse weather conditions [199]. To address this, the response of nonconventional aircraft concepts to these disturbances has been studied recently by different authors, including flying-wing configurations $[8,147,200]$, blended-wing-body airliners [163], and flexible HALE platforms [134]. In fact, both the sizing of the structure and the design of flight control systems (FCS) are intimately linked to the response of the aircraft to atmospheric disturbances.

For the certification of large commercial aeroplanes the airworthiness authorities require clearance of continuous turbulence and tuned discrete-gusts [201,202], which affect fatigue life of structural members and the maximum stresses they are subject to. These are evaluated through an idealisation of the disturbance, namely a power spectral density (PSD) approach for continuous random turbulence and "1-cos" pulses of different lengths and intensities for discrete gusts. Methods of different fidelity might be employed to represent the aircraft dynamics, but they customarily include gust-penetration effects and model the tail plane. In both continuous and discrete analysis, vertical and lateral disturbances are considered for the relevant mass configurations, flight levels, and Mach numbers. The gust impact on manoeuvres might also be accounted for. In all cases, the load alleviation due to the rigidbody motions of the vehicle needs to be considered [203].

Continuous turbulence is generally analysed in the frequency domain, assuming isotropy and a statistical Gaussian probability distribution, where the PSD is represented by Dryden [204] or von Kármán $[205,206]$ spectra. The PSD and root-mean-square values of the response are then obtained through the relevant aircraftdynamics model, which as based in the frequency domain, needs to be linear. Alternatively, rational approximations to the longitudinal and transverse spectra are also possible, which allow time histories of the turbulence to be obtained [207], and thus evaluation of the response in the time domain including nonlinearities, such as those of FCS or due to large deformations.

Discrete-gust response is studied in the time domain, and models based on strip theory, for instance, allow nonlinearities to be incorporated. In contrast, the most common approach in industry is based on the DLM, which provides the aerodynamic influence coefficients as a function of the reduced frequency, and is therefore linear. In this case, a state-space time-domain representation is obtained via a rational function approximation (see Section 3.1.1). Further nonlinearities arise if the flight conditions are in the transonic regime, and in this case one would need to resort to CFD analysis [208]. Irrespective of the model used, discrete-gust analysis requires a tuning process in which the critical gust length of the "1-cos" pulse is determined for each set of flight conditions on a set of "Interesting Quantities" (e.g., root bending moment, shear force at engine mounts, etc.). The

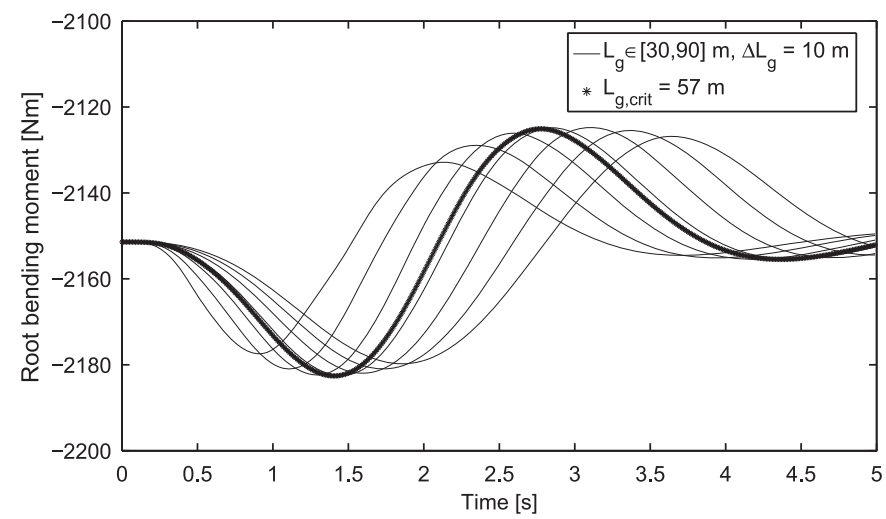

Fig. 20. Root bending moment of the main wing of the test aircraft undergoing " 1 cos" gust disturbances of different lengths. Critical gust length is $L_{g \text {,crit }}=57 \mathrm{~m}$. $V_{g 0}=0.01 V_{\infty}$ and nominal conditions.

UVLM is exercised here to show how this discrete-gust tuning can be performed on a fully-flexible vehicle.

The inclusion of gust disturbances in the standard UVLM is straightforward, and the implementation of a discrete " 1 -cos" pulse is described, for instance, by Wang et al. [8]. The gustinduced velocities affect the non-penetration boundary condition at the collocation points, Eqs. (5) and (9). They also modify the expression for the aerodynamic loads, Eq. (13), through the local aerodynamic velocities, $\dot{\zeta}_{b}$. Finally, the gust will propagate into the wake if this is considered fully force-free, hence affecting the local velocities at the wake grid, $\boldsymbol{V}$ in Eq. (10), and changing its rolled-up shape. The linear state-space formulation described in Section 5.3 can also incorporate prescribed gust profiles. In this case, the open-loop equivalent of Eq. (37) is considered, i.e.,

$E_{\text {sys }} \Delta \boldsymbol{x}^{n+1}=A_{\text {sys }} \Delta \boldsymbol{x}^{n}+B_{d} \Delta \boldsymbol{u}_{d}^{n}$.

The gust-induced velocities affect the aerodynamic states through the non-penetration boundary condition, and the elastic and rigid-body states through the aerodynamic loads, Eq. (34). Recall that this linearised formulation does not include a forcefree wake, which is frozen at the equilibrium conditions.

Only the case of a vertical stationary discrete "1-cos" pulse will be considered here. The spanwise distribution is assumed constant. At a penetration length through the disturbance, $x_{g}(t)$, the vertical gust velocity is given by

$V_{g}(t)=\frac{1}{2} V_{g 0}\left[1-\cos \left(\frac{2 \pi x_{g}}{L_{g}}\right)\right], \quad 0 \leq x_{g} \leq L_{g}$,

where $V_{g 0}$ is the gust intensity, and $L_{g}$ its length. At time step $n$, the column vector with the gust velocities at all collocation points, $\boldsymbol{V}_{g}^{n}$, coincides with $\Delta \boldsymbol{u}_{d}^{n}$ in Eq. (38), where the relationship between the penetration length and time is given by

$x_{g}=V_{\infty} t^{n}-x_{k}$,

and where $x_{k}$ is the coordinate of the $k$ th collocation point on the body-fixed frame, assuming the origin of this frame is located at the edge of the incident gust at the beginning of the motion.

In order to determine the critical gust length, the root bending moment is computed. The model aircraft, defined in Fig. 9 and Table 2, is considered, with nominal conditions of $V_{\infty}=25 \mathrm{~m} / \mathrm{s}$, $\sigma=1$, and $d_{p l}=0 \mathrm{~m}$, and including all aerodynamic, structural, and rigid-body states. Fig. 20 presents the root bending moment of the main wing as the vehicle undergoes "1-cos" gusts of different lengths. Results have been obtained via the open-loop linear state-space equations, Eq. (38). A small gust intensity of $V_{g 0}=0.01 V_{\infty}$ is simulated, so that the results remain linear. Gust lengths from $30 \mathrm{~m}$ to $90 \mathrm{~m}$ have been evaluated, in increments of 
a

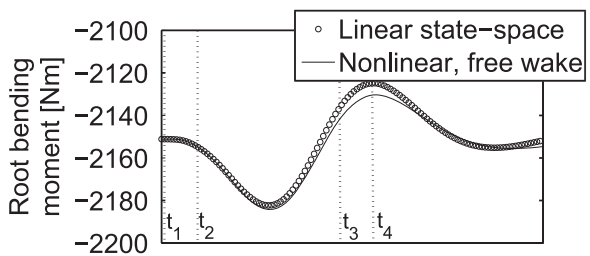

C

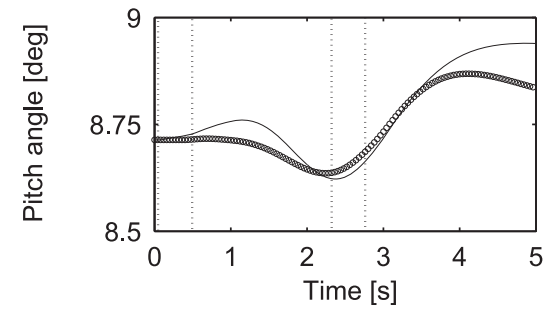

b

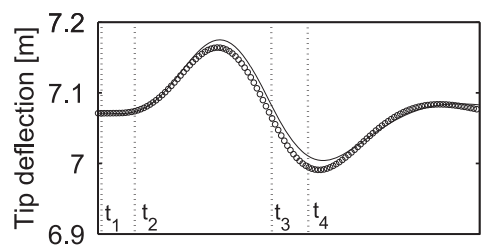

d

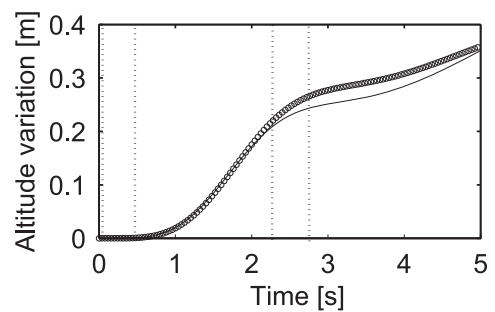

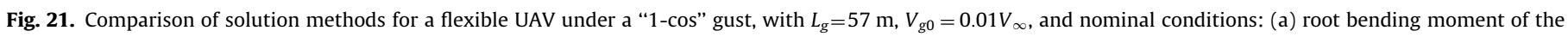
wing, (b) tip deflection of the wing, (c) pitch angle of the aircraft, and (d) altitude variation.

a

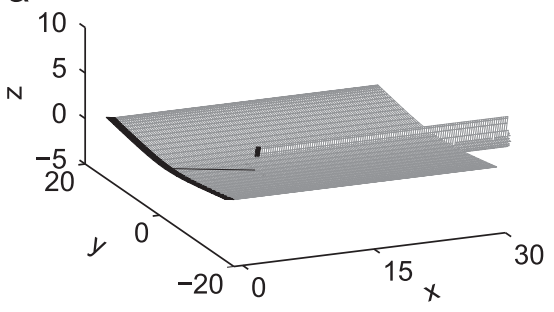

C

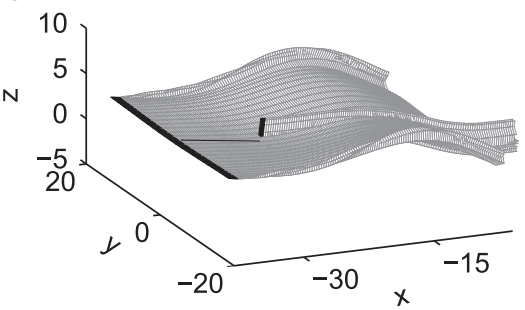

b

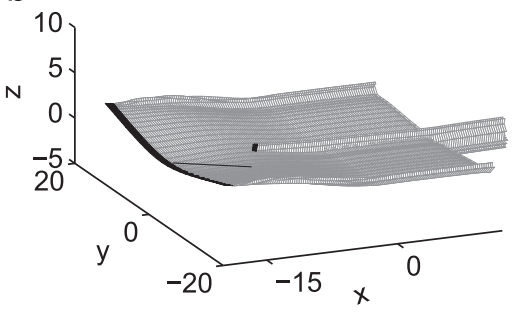

d

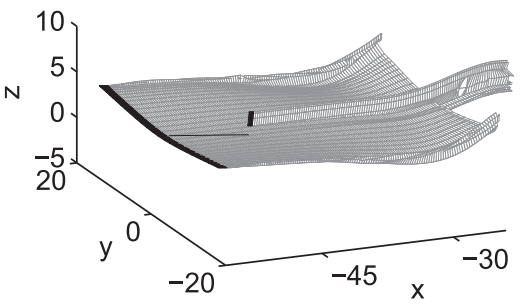

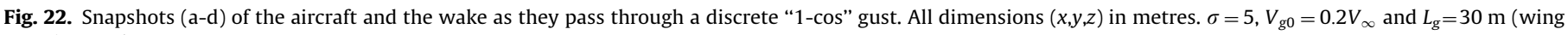
span is $32 \mathrm{~m}$ ).

$10 \mathrm{~m}$, and after refining to increments of $1 \mathrm{~m}$, the critical gust length is found to be $L_{g, c r i t}=57 \mathrm{~m}$, or 57 wing-chord lengths. It can be observed that the initial root bending moment, which corresponds to the deformed wing of the trimmed vehicle, is significant. This is caused by the large deformations of the wing at equilibrium, $44 \%$ of the semi-span. The plots illustrate the typical trend for a flexible member due to a gust: the wing experiences up-bending motions as the gust velocity increases, and then bends downwards as the gust is left behind.

As a next step, the critical gust length is tested in a fully nonlinear simulation, including a free wake. The gust intensity is kept at the above level in order to guarantee small deformations around the equilibrium configuration. This allows the assumption of a frozen wake in the state-space formulation to be assessed. Fig. 21 displays the comparison. Vertical lines have been added on the plots, and they represent, from left to right: (1) main wing reaches the origin of the stationary gust $\left(t_{1}\right)$; (2) tail reaches gust $\left(t_{2}\right)$; (3) main wing leaves gust $\left(t_{3}\right)$; and (4) tail leaves gust $\left(t_{4}\right)$

Fig. 21(a) presents the root bending moment of the wing, and Fig. 21(b) its tip deflection. The agreement is reasonably good, particularly when the critical load and maximum tip deflection occur, which is the point of maximum interest. However, the discrepancy is higher on the second peak, which is mainly caused by the different attitude of the aircraft. Fig. 21(c) shows the pitch angle of the vehicle. Both solution methodologies provide different results, but note that the variations around the trim value are small. Finally, Fig. 21(d) depicts the variation in altitude experienced by the aircraft, which climbs on once the gust is left behind until a new equilibrium is reached. This variation in altitude introduces an alleviation factor into the gust loads: the aircraft climbs when hit by the gust, hence inducing aerodynamic forces that resist the deformation.

Linear gust load analysis using the UVLM under the prescribed-wake assumption yields results of the same fidelity as those obtained by the DLM, with the advantage that the direct state-state solution does not require a rational-function approximation (a potential source of errors). The results above confirm that the prescribed wake assumption can give a good approximation to predict linear gust response, capturing the critical load conditions. Here a very flexible vehicle has been simulated undergoing a gust of small intensity, but the methodology would be equally valid for a stiffer aircraft and larger gust intensities. 
Overall, the deformations in the example remain in the linear regime (around the nonlinear static equilibrium).

Since structural deformations are small, the free wake seems to be responsible for the mismatch observed with the fully nonlinear solver. The force-free wake will have an impact on the load distribution of the main wing, but its effect will be more prominent on the tail. As the wake rolls up and the gust propagates into it, the tail "sees" a modified shape, and the aerodynamic interference will therefore vary. The tail is considered rigid in these simulations, but the wake effect is likely to be visible in the pitch rate of the aircraft. This, in turn, will affect rigid-body motions, elastic deformations, and aerodynamic loads, explaining the disagreements in Fig. 21. On the other hand, note that the variation in aerodynamic loads due to the free wake will cause the linear model not to represent the exact trim at the initial conditions, hence introducing a further source of error.

Finally, in order to illustrate the gust convection downstream, Fig. 22 presents several snapshots of the vehicle (wing, fuselage, tail) and the corresponding wakes. For visualisation purposes, a gust with $V_{g 0}=0.2 V_{\infty}$ and $L_{g}=30 \mathrm{~m}$ has been implemented, and $\sigma=5$ has been considered. The latter yields a static tip deflection of $z_{\text {tip }}=1.11 \mathrm{~m}$ at trim conditions. The propagation of the gust over the wakes of the main wing and tail can be identified, and the roll up of the wake at the tips is also revealed.

The aircraft pitches up first as the gust hits the main wing, and this initial climb leads to a maximum change in altitude of $3.54 \mathrm{~m}$. The effect of the gust on the tail provokes a downward pitching moment, and hence the aircraft descends next. This can be inferred from Fig. 22 by the portion of the vertical fin immersed below the wake of the main wing-more fin is visible as the vehicle pitches down. During this motion, the wing also deforms under the effect of the gust, first bending upwards, and then downwards. The maximum tip deflection experienced by the wing is $2.12 \mathrm{~m}$ and occurs at $V_{\infty} t / L_{g}=0.70$, whereas the minimum deflection is $0.14 \mathrm{~m}$ at $V_{\infty} t / L_{g}=1.28$. Obviously, due to the unsteadiness of the problem, the aerodynamic loading is not instantaneous and there is a lag between the actual gust encounter and the subsequent resultant motion and deformations. Note that the rigid-body climbing motion of the aircraft alleviates quite substantially the excursions experienced by the wing, and that these deformations, in turn, mitigate the maximum altitude variation undergone by the vehicle.

\section{Conclusions and outlook}

The steady vortex-lattice method is a well-known incompressible potential-flow technique that has been, and still is, extensively used for low-speed-flight calculations. In contrast, the unsteady version has been largely overlooked in fixed-wing aircraft dynamics modelling, and has been mostly exercised in other disciplines, such as rotorcraft, wind turbines or flapping-wing vehicle analysis. The double lattice is the reigning tool in linear aeroelasticity, and 2D strip theory has been favoured recently in the conceptual design of large flexible HALE UAVs. However, the UVLM represents a suitable candidate in many situations, and this paper has emphasised its applicability in aeroelasticity and flight dynamics at low speeds. Scenarios where it is not only a powerful alternative, but even a necessary one, have been highlighted, hence illustrating its full potential. Indeed, it could be argued that the savings in CPU time obtained by the DLM are no longer relevant and that the linear state-space UVLM, which is more general, could replace it in virtually all cases.

The UVLM is governed by Laplace's equation, which is linear, and thus is constrained to the subsonic regime with negligible viscous effects. However, the enforcement of the boundary conditions on the deformed geometry allows geometrically nonlinear deflections of the lifting surfaces to be captured. In its standard formulation the UVLM models a force-free wake, incorporating a further nonlinearity into the problem. In turn, the linearised version of the equations offers the same level of fidelity as state-of-the-art tools in aeroelasticity, and caters for in-plane motions and static nonlinear wing excursions. The latter are likely to become a common feature of flexible HALE vehicles, but they might also arise in the certification process of commercial aircraft, due to the high load factors under which they must operate without damage.

Integrating the aerodynamics with structural and rigid-body models, a full description of the dynamics of a flexible vehicle is accomplished. Here a geometrically nonlinear beam has been implemented, but models of various degrees of complexity, including descriptions based on modal analysis, could be appended instead. The resulting simulation framework allows analyses such as aircraft trimming, linear stability evaluation, and linear or nonlinear gust response to be performed. Numerical studies focusing on these capabilities have been presented, illustrating the flutter of a T-tail and the open-loop characteristics of a representative HALE configuration.

A key characteristic evidenced by the results is that including nonlinear effects may lead to unexpected trends. The flutter speed of a clamped flexible wing has been shown to significantly decrease with flexibility, and undesirable couplings between elastic and rigid-body oscillatory modes have been identified. However, the stiffness reduction of airframe structures does not necessarily have a detrimental impact. In fact, the opposite is true in some cases. For example, the stability envelope of the freeflying aircraft may increase with wing deformations: the static margins for payload location are widened, and the spiral mode becomes stable due to the increased effective dihedral.

Time marching the fully nonlinear system is a relatively lengthy process, but it is sometimes necessary. In these cases, computing the local velocities on the free wake is the main contributor to the total simulation cost. Practical solutions to this problem exist though, such as parallelisation of the code or implementation of acceleration algorithms whereby the details of the wake far-field are approximated.

In contrast, the monolithic state-space assembly obtained through the linearisation of the equations proves extremely efficient, and each point of the parametric space takes just a few seconds to evaluate in a desktop computer. In addition, this formulation casts in the same analysis static and dynamic stability, enables the straightforward implementation of reduced-order modelling techniques, and is ideally suited for advanced control synthesis methods. The prescribed-wake assumption provides an acceptable level of accuracy and insight, before the closed-loop solution is then tested on the nonlinear solver. Finally, the state-space formulation is also expected to constitute a valuable tool to address some of the pressing issues industry is facing, such as multidisciplinary optimisation and uncertainty modelling.

CFD simulations still incur computational expenses that are often not permissible for routine analysis of flexible-aircraft dynamics, or require a level of detail which is simply not available at the conceptual design stage. Until the day arrives when these high-fidelity tools become practical for those tasks, the unsteady vortex-lattice method should be contemplated as an outstanding aerodynamics model, and employed alongside existing methods to drive aeronautical research.

\section{Acknowledgements}

Part of this work was carried out during a placement of the first author at the Active Aeroelasticity and Structures Research 
Laboratory, University of Michigan, led by Professor C.E.S. Cesnik. His support, advice, and guidance are greatly appreciated. The authors would also like to thank Henrik Hesse of Imperial College London for his contributions on the flexible-body dynamics model. Finally, Joseba Murua gratefully acknowledges the financial support of the Department of Education, Universities and Research of the Basque Government.

\section{References}

[1] Albano E, Rodden WP. A doublet lattice method for calculating lift distributions on oscillating surfaces in subsonic flow. AIAA Journal 1969;7(2): 279-85, http://dx.doi.org/10.2514/3.5086.

[2] Kier TM. Comparison of unsteady aerodynamic modelling methodologies with respect to flight loads analysis. In: AIAA atmospheric flight mechanics conference, AIAA 2005-6027, San Francisco, CA, USA, 2005.

[3] Lucia DJ. The SensorCraft configurations: a non-linear aeroservoelastic challenge for aviation. In: 46th AIAA/ASME/ASCE/AHS/ASC structures, structural dynamics, and materials conference, Austin, TX, United States, 2005. pp. 1768-74, AIAA Paper 2005-1943.

[4] Shearer CM, Cesnik CES. Nonlinear flight dynamics of very flexible aircraft Journal of Aircraft 2007;44(5):1528-45, http://dx.doi.org/10.2514/1.27606.

[5] van Schoor MC, van Flotow AH. Aeroelastic characteristics of a highly flexible aircraft. Journal of Aircraft 1990;27(10):901-8, http://dx.doi.org/ $10.2514 / 3.45955$.

[6] Drela M. Integrated simulation model for preliminary aerodynamic, structural, and control-law design of aircraft. In: 40th AIAA structures, structura dynamics and materials conference, AIAA 99-1934, St. Louis, MO, USA 1999. pp. 1644-56.

[7] Baldelli DH, Chen PC, Panza J. Unified aeroelastic and flight dynamic formulation via rational function approximations. Journal of Aircraft 2006;43(3):763-72, http://dx.doi.org/10.2514/1.16620.

[8] Wang Z, Chen PC, Liu DD, Mook DT. Nonlinear-aerodynamics/nonlinearstructure interaction methodology for a high-altitude long-endurance wing Journal of Aircraft 2010;47(2):556-66, http://dx.doi.org/10.2514/1.45694.

[9] Patil MJ, Hodges DH, Cesnik CES. Nonlinear aeroelastic analysis of complete aircraft in subsonic flow. Journal of Aircraft 2000;37(5):753-60, http://dx.doi.o $\mathrm{rg} / 10.2514 / 2.2685$

[10] Patil MJ, Hodges DH, Cesnik CES. Nonlinear aeroelasticity and flight dynamics of high-altitude long-endurance aircraft. Journal of Aircraft 2001;38(1):88-94, http://dx.doi.org/10.2514/2.2738.

[11] Meirovitch L, Tuzcu I. Unified theory for the dynamics and control of maneuvering flexible aircraft. AIAA Journal 2004;42(4):714-27, http://dx.doi.org/10.2514/ 1.1489.

[12] $\overline{\text { Romeo }}$ G, Frulla G, Marzocca P, Tuzcu I. Non-linear aeroelastic modeling and experiments of flexible wings. In: 47th AIAA/ASME/ASCE/AHS/ASC structures, structural dynamics, and materials conference. AIAA 2006-2186, Newport, RI, USA, 2006

[13] Palacios R, Cesnik CES, Reichenbach EY. Re-examined structural design procedures for very flexible aircraft. In: International forum on aeroelasticity and structural dynamics, Stockholm, Sweden, 2007.

[14] Demasi L, Livne E. Dynamic aeroelasticity of structurally nonlinear config urations using linear modally reduced aerodynamic generalized forces. AIAA Journal 2009;47(1):71-90, http://dx.doi.org/10.2514/1.34797.

[15] Su W, Cesnik CES. Nonlinear aeroelasticity of a very flexible blended-wingbody aircraft. Journal of Aircraft 2010;47(5):1539-53, http://dx.doi.org/ $10.2514 / 1.47317$.

[16] Zhao Z, Ren G. Multibody dynamic approach of flight dynamics and nonlinear aeroelasticity of flexible aircraft. AIAA Journal 2011;49(1):41-54 http://dx.doi.org/10.2514/1.45334.

[17] Palacios R, Murua J, Cook R. Structural and aerodynamic models in the nonlinear flight dynamics of very flexible aircraft. AIAA Journal 2010;48(11):2559-648, http://dx.doi.org/10.2514/1.52446.

[18] Murua J, Palacios R, Graham JMR. Assessment of wake-tail interference effects on the dynamics of flexible aircraft. AIAA Journal 2010;50(7):15751585, http://dx.doi.org/10.2514/1.J051543.

[19] Maute K, Nikbay M, Farhat C. Coupled analytical sensitivity analysis and optimization of three-dimensional nonlinear aeroelastic systems. AIAA Journal 2001;39(11):2051-61, http://dx.doi.org/10.2514/2.1227.

[20] Geuzaine P, Brown G, Harris C, Farhat C. Aeroelastic dynamic analysis of a full F-16 configuration for various flight conditions. AIAA Journal 2003;41(3): 363-71, http://dx.doi.org/10.2514/2.1975.

[21] Garcia JA. Numerical investigation of nonlinear aeroelastic effects on flexible high-aspect-ratio wings. Journal of Aircraft 2005;42(4):1025-36 http://dx.doi.org/10.2514/1.6544.

[22] Batchelor GK. An introduction to fluid dynamics. Cambridge University Press; 1967.

[23] Rosenhead L. Formation of vortices from a surface of discontinuity. Proceedings of the Royal Society of London. Series A: Mathematical, Physical and Engineering Sciences 1931;134:170-92 〈http://www.jstor.org/stable/ 95835 > Last retrieved: 15 Jan 2012.
[24] Lavrent'ev MA. On a construction of a flow that engulfs an arc of a given form. Proceedings of the Central Institute of Aerohydrodynamics 1932;113:3-56.

[25] Falkner VM. The calculation of aerodynamic loading on surfaces of any shape. In: Aeronautical research council reports and memoranda; 1943.

[26] Watkins CE, Runyan HL, Cunninghman HJ. A systematic kernel function procedure for determining aerodynamic forces on oscillating or steady finite wings at subsonic speeds, NASA Report R-48; 1959.

[27] Stark VJE. Aerodynamic forces on a combination of a wing and a fin oscillating in subsonic flow. SAAB TN 1964;54.

[28] Davies DE. Generalised aerodynamic forces on a T-tail oscillating in subsonic flow. In: Aeronautical research council reports and memoranda. No. 3422; 1964.

[29] Vivian HT, Andrew LV. Unsteady aerodynamics for advanced configurations. Part I: application of the subsonic kernel function to nonplanar lifting surfaces, USAF FDL-TDR-64-452; 1965.

[30] Laschka B, Schmidt H. Unsteady aerodynamic forces on coplanar lifting surfaces in subsonic flow (wing-horizontal tail interference), Jahrbuch 1967 der WGLR; 1967.

[31] Berman JH, Shyprykevich P, Smedfeld JB, Kelly RF. Unsteady aerodynamic forces for general wing/control-surface configurations in subsonic flow, USAF FDL-TR-67-117; 1968.

[32] Hedman SG. Vortex lattice method for calculation of quasi steady state loadings on thin elastic wings in subsonic flow. The Aeronautical Research Institute of Sweden. Report 105; 1965.

[33] Vortex-Lattice Utilization, NASA SP-405, a workshop held at Langley Research Center, Hampton, VA, USA (17-18 May 1976).

[34] Margason RJ, Lamar JE. Vortex-lattice FORTRAN program for estimating subsonic aerodynamic characteristics of complex planforms. NASA TN D-6142; 1971.

[35] Lamar JE, Herbert HE. Production version of the extended NASA-Langley Vortex Lattice FORTRAN computer program, vol. I. User's guide, NASA TM 83303; 1982.

[36] Herbert HE, Lamar JE. Production version of the extended NASA-Langley Vortex Lattice FORTRAN computer program, vol. II. Source code, NASA TM 83304; 1982.

[37] James RM. On the remarkable accuracy of the vortex lattice method. Computer Methods in Applied Mechanics and Engineering 1972;1(1):59-79, http://dx.doi.org/10.1016/0045-7825(72)90021-7.

[38] Hough GR. Remarks on vortex-lattice methods. Journal of Aircraft 1973;10(5):314-7, http://dx.doi.org/10.2514/3.44370.

[39] DeJarnette FR. Arrangement of vortex lattices on subsonic wings. In: Vortexlattice utilization. Hampton, VA, USA: Langley Research Center; 1976.

[40] Hunt B. The mathematical basis and numerical principles of the boundary integral method for incompressible potential flow over 3-D aerodynamic configurations. In: Numerical methods in applied fluid dynamics. Academic Press Inc.; 1980. Based on the proceedings of the conference on numerical methods in applied fluid dynamics, University of Reading; 1978.

[41] Rusak Z, Seginer A, Wasserstrom E. Convergence characteristics of a vortexlattice method for nonlinear configuration aerodynamics. Journal of Aircraft 1985;22(9):743-9, http://dx.doi.org/10.2514/3.45196.

[42] Mook DT, Nayfeh AH. Application of the vortex-lattice method to highangle-of-attack subsonic aerodynamics. SAE paper no. 851817; October 1985.

[43] Polhamus EC. A concept of the vortex lift on sharp edge delta wings based on a leading-edge suction analogy. NASA-TN 3767; 1966.

[44] Polhamus EC. Prediction of vortex-lift characteristics by a leading-edge suction analogy. Journal of Aircraft 1971;8(4):193-9, http://dx.doi.org/ $10.2514 / 3.44254$.

[45] Lan CE. A quasi-vortex-lattice method in thin wing theory. Journal of Aircraft 1974;11(9):518-27, http://dx.doi.org/10.2514/3.60381.

[46] Lamar JE, Gloss BB. Subsonic aerodynamic characteristics of interacting lifting surfaces with separated flow around sharp edges predicted by a vortex-lattice method. NASA TN D-7921; 1975.

[47] Polhamus EC. Application of slender wing benefits to military aircraft. In: AIAA Wright brothers lecture. AIAA 83-2566; 1983.

[48] Fiddes SP, Gaydon JH. A new vortex lattice method for calculating the flow past yacht sails. Journal of Wind Engineering and Industrial Aerodynamics 1996;63(1-3):35-59, http://dx.doi.org/10.1016/S0167-6105(96)00068-2.

[49] Kay J, Mason WH, Durham W, Lutze F, Benoliel A. Control authority issues in conceptual design: critical conditions, estimation methodology, spreadsheet assessment, trim and bibliography. Technical report VPI-Aero-200, Virginia Polytechnic Institute and State University, Department of Aerospace and Ocean Engineering; November 1993.

[50] Melin T. User's guide and reference manual for Tornado, Royal Institute of Technology (KTH), Department of aeronautics, Stockholm, Sweden <http:// www.redhammer.se/tornado/index.html $>$ Last retrieved: 15 January 2012; December 2000.

[51] Drela M, Youngren H. AVL 3.30 User primer , 〈http://web.mit.edu/drela/ Public/web/avl/ $>$ Last retrieved: 15 January 2012; 2010.

[52] Rossow VJ, Tinling BE. Research on aircraft/vortex-wake interactions to determine acceptable level of wake intensity. Journal of Aircraft 1988;25(6):481-92, http://dx.doi.org/10.2514/3.45610.

[53] Elzebda JM, Mook DT, Nayfeh AH. Numerical simulation of steady and unsteady, vorticity-dominated aerodynamic interference. Journal of Aircraft 1994;31(5):1031-6, http://dx.doi.org/10.2514/3.46606. 
[54] Rossow VJ. Validation of vortex-lattice method for loads on wings in liftgenerated wakes. Journal of Aircraft 1995;32(6):1254-62, http://dx.doi.org/ $10.2514 / 3.46872$

[55] Haghighat S, Liu HHT, Martins JRRA. Application of robust control design techniques to the aeroservoelastic design optimization of a very flexible UAV wing. In: 13th AIAA/ASMO multidisciplinary analysis optimization conference. AIAA 2010-9123. Forth Worth, TX, USA; 2010.

[56] Rodden WP, Taylor PF, McIntosh Jr. SC. Further refinement of the subsonic doublet-lattice method. Journal of Aircraft 1998;35:720-26 http://dx.doi. org/10.2514/2.2382.

[57] Kálmán TP, Rodden WP, Giesing JP. Application of the doublet-lattice method to nonplanar configurations in subsonic flow. Journal of Aircraft 1971;8:406-13, http://dx.doi.org/10.2514/3.59117.

[58] Giesing JP, Kálmán TP, Rodden WP. Subsonic unsteady aerodynamics for general configurations. Part 2: application of the doublet-lattice method and the method of images to lifting-surface/body interference. USAF FDLTR-5, Part II; 1972.

[59] Rodden WP, Baker ML. Improving the convergence of the doublet-lattice method through tip corrections. In: International forum on aeroelasticity and structural dynamics, Williamsburg, VA, USA; 1999.

[60] Wright JR, Cooper JE. Introduction to aircraft aeroelasticity and loads. John Wiley \& Sons Ltd; 2007.

[61] Hess JL. Calculation of potential flow about arbitrary three-dimensional lifting bodies. In: Final technical report MDC J5679-01. Douglas Aircraft Co., Long Beach, CA, USA; October 1972.

[62] Katz J, Plotkin A. Low-speed aerodynamics, 2nd ed. Cambridge Aerospace Series. Cambridge University Press; 2001.

[63] Belotserkovskii SM. Study of the unsteady aerodynamics of lifting surfaces using the computer. Annual Review of Fluid Mechanics 1977;9:469-94, http://dx.doi.org/10.1146/annurev.fl.09.010177.002345.

[64] Rehbach C. Numerical calculation of three dimensional unsteady flows with vortex sheets. La Recherche Aérospatiale September-October 1977. p. 289-98. Also AIAA 1978-111.

[65] Atta EH, Kandil OA, Mook DT, Nayfeh AH. Unsteady aerodynamic loads on arbitrary wings including wing-tip and leading-edge separation. AIAA 1977-156; 1977.

[66] Konstadinopoulos P, Thrasher DF, Mook DT, Nayfeh AH, Watson L. A vortexlattice method for general unsteady aerodynamics. Journal of Aircraft 1985;22(1):43-9, http://dx.doi.org/10.2514/3.45078.

[67] Levin D, Katz J. Vortex-lattice method for the calculation of nonsteady separated flow over delta wings. Journal of Aircraft 1981;18:1032-7, http://dx.doi.org/10.2514/3.57596.

[68] Katz J. Lateral aerodynamic of delta wings with leading-edge separation. AIAA Journal 1984;22(3):323-8, http://dx.doi.org/10.2514/3.8395.

[69] Fritz TE, Long LN. Object-oriented unsteady vortex lattice method for flapping flight. Journal of Aircraft 2004;41(6):1275-90, http://dx.doi.org/ $10.2514 / 1.7357$.

[70] Willis DJ, Peraire J, White JK. A combined pFFT-multipole tree code, unsteady panel method with vortex particle wakes. International Journal for Numerical Methods in Fluids 2007;53:1399-422, http://dx.doi.org/ $10.1002 /$ fld 1240

[71] Stanford BK, Beran PS. Analytical sensitivity analysis of an unsteady vortex lattice method for flapping wing optimization. Journal of Aircraft 2010;47(2): 647-62, http://dx.doi.org/10.2514/1.46259.

[72] Röttgermann A, Behr R, Schöttl C, Wagner S. Calculation of blade-vortex interaction of rotary wings in incompressible flow by an unsteady vortexlattice method including free wake analysis. Notes on Numerical Fluid Mechanics 1992;33:153-66.

[73] Wie SY, Lee S, Lee DJ. Potential panel and time-marching free-wake coupling analysis for helicopter rotor. Journal of Aircraft 2009;46(3): 1030-41, http://dx.doi.org/10.2514/1.40001.

[74] Simoes FJ, Graham JMR. Application of a free vortex wake model to a horizontal axis wind turbine. Journal of Wind Engineering and Industrial Aerodynamics 1992;39(1-3):129-38, http://dx.doi.org/10.1016/01676105(92)90539-M.

[75] Coton FN, Wang T. The prediction of horizontal axis wind turbine performance in yawed flow using an unsteady prescribed wake model. Proceedings of the Institution of Mechanical Engineers, Part A: Journal of Power and Energy 1999; 213:33-43 http://dx.doi.org/10.1243/0957650991537419.

[76] Grant I, Mo M, Pan X, Parkin P, Powell J, Reinecke H, et al. An experimental and numerical study of the vortex filaments in the wake of an operational, horizontal-axis, wind turbine. Journal of Wind Engineering and Industrial Aerodynamics 2000;85(2):177-89, http://dx.doi.org/10.1016/S01676105(99)00139-7.

[77] Pesmajoglou SD, Graham JMR. Prediction of aerodynamic forces on horizontal axis wind turbines in free yaw and turbulence. Journal of Wind Engineering and Industrial Aerodynamics 2000;86(1):1-14, http://dx.doi.org/10.1016 S0167-6105(99)00125-7.

[78] Rados K, Larsen G, Barthelmie R, Schlez W, Lange B, Schepers G, Hegberg T, Magnisson M. Comparison of wake models with data for offshore wind farms. Wind Engineering 2001;25(5):271-80, http://dx.doi.org/10.1260/ 030952401760177846.

[79] Hansen MOL, Sørensen JN, Voutsinas S, Sørensen N, Madsen HA. State of the art in wind turbine aerodynamics and aeroelasticity. Progress in Aerospace Sciences 2006;42(4):285-330, http://dx.doi.org/10.1016/j.paerosci.2006. $\underline{10.002}$
[80] Døssing M. Vortex lattice modelling of winglets on wind turbine blades. Master's thesis. Risø National Laboratory, Technical University of Denmark, Roskilde, Denmark; August 2007.

[81] Karkehabadi R. Aerodynamic interference of a large and a small aircraft Journal of Aircraft 2004;41(6):1424-9, http://dx.doi.org/10.2514/1.4570.

[82] Sequeira CJ, Willis DJ, Peraire J. Comparing aerodynamic models for numerical simulation of dynamics and control of aircraft. In: 44th AIAA aerospace sciences meeting and exhibit. AIAA 2006-1254, Reno, NV, USA; 2006.

[83] Hall BD, Mook DT, Nayfeh AH, Preidikman S. Novel strategy for suppressing the flutter oscillations of aircraft wings. AIAA Journal 2001;39(10): 1843-50, http://dx.doi.org/10.2514/2.1190.

[84] Wang Z, Chen PC, Liu DD, Mook DT, Patil MJ. Time domain nonlinear aeroelastic analysis for HALE wings. In: 47th AIAA/ASME/ASCE/AHS/ASC structures, structural dynamics, and materials conference. AIAA 2006-1640, Newport, RI, USA; 2006.

[85] Stanford B, Beran P. Formulation of analytical design derivatives for nonlinear unsteady aeroelasticity. AIAA Journal 2011;49(3):598-610, http://dx.doi.org/ $10.2514 / 1.53411$.

[86] Obradovic B, Subbarao K. Modeling of flight dynamics of morphing-wing aircraft. Journal of Aircraft 2011;48(2):391-402, http://dx.doi.org/10.2514/ 1.C000269.

[87] Hall KC. Eigenanalysis of unsteady flows about airfoils, cascades, and wings AIAA Journal 1994;32(12):2426-32, http://dx.doi.org/10.2514/3.12309.

[88] Dowell EH, Hall KC. Eigenmode analysis in unsteady aerodynamics: Reduced order models. Applied Mechanics Reviews 1997;50(6):371-87, $\underline{\mathrm{h}}$ ttp://dx.doi.org/10.1115/1.3101718.

[89] Tang D, Dowell EH, Hall KC. Limit cycle oscillations of a cantilevered wing in low subsonic flow. AIAA Journal 1999;37(3):364-71, http://dx.doi.org/ $10.2514 / 2.717$

[90] Tang D, Kholodar D, Juang J-N, Dowell EH. System identification and proper orthogonal decomposition method applied to unsteady aerodynamics. AIAA Journal 2001;39(8):1569-76, http://dx.doi.org/10.2514/2.1482.

[91] Juang J-N, Kholodar D, Dowell EH. System identification of a vortex lattice aerodynamic model. AIAA Journal 2002;40(6):1187-96, http://dx.doi.org/ $10.2514 / 2.1770$.

[92] Voutsinas SG. Vortex methods in aeronautics: how to make things work International Journal of Computational Fluid Dynamics 2006;20(1):3-18, http:/ /dx.doi.org/10.1080/10618560600566059.

[93] Greengard L, Rohklin V. A fast algorithm for particle simulations. Journal of Computational Physics 1987;73:325-84, http://dx.doi.org/10.1016/00219991(87)90140-9.

[94] Bagai A, Leishman JG. Adaptive grid sequencing and interpolation for rotor fee-wake analyses. AIAA Journal 1998;36(9):1593-602, http://dx.doi.org/ $10.2514 / 2.585$

[95] Leishman JG. Principles of helicopter aerodynamics, 2nd ed. Cambridge Aerospace Series, Cambridge University Press; 2006.

[96] Leishman JG, Bhagwat MJ, Bagai A. Free-vortex filament methods for the analysis of helicopter rotor wakes. Journal of Aircraft 2002;39(5):759-75, ht tp://dx.doi.org/10.2514/2.3022.

[97] Saffman PG, Baker GR. Vortex interactions. Annual Review of Fluid Mechanics 1979;11:95-121, http://dx.doi.org/10.1146/annurev.fl.11.010179. 000523.

[98] Sarpkaya T. Computational methods with vortices-the 1988 Freeman scholar lecture. Journal of Fluids Engineering 1989;111(1):5-52 http://dx.doi.org/10. $1115 / 1.3243601$.

[99] Cottet G-H, Koumoutsakos PD. Vortex methods: theory and practice. Cambridge University Press; 2000.

[100] Quackenbush TR, Bliss DB, Wachspress DA. New free-wake analysis of rotorcraft hover performance using influence coefficients. Journal of Aircraft 1989;26(12):1090-7, http://dx.doi.org/10.2514/3.45885.

[101] Vatistas GH, Kozel V, Mih WC. A simpler model for concentrated vortices Experiments in Fluids 1991;11(1):73-6, http://dx.doi.org/10.1007/ BF00198434.

[102] Rahier G, Delrieux Y. Blade-vortex interaction noise prediction using a rotor wake roll-up model. Journal of Aircraft 1997;34(4):522-30, http://dx.doi.or g/10.2514/2.2204.

[103] Conlisk AT. Modern helicopter aerodynamics. Annual Review of Fluid Mechanics 1997;29:515-67, http://dx.doi.org/10.1146/annurev.fluid.29.1.515.

[104] Maskew B. Subvortex technique for the close approach to a discretized vortex sheet. Journal of Aircraft 1977;14(2):188-93, http://dx.doi.org/ $10.2514 / 3.44582$

[105] Bramesfeld G, Maughmer MD. Relaxed-wake vortex-lattice method using distributed vorticity elements. Journal of Aircraft 2008;45(2):560-8 http://dx.doi.org/10.2514/1.31665.

[106] Rehbach C. Calcul d'ecoulements autour d'ailes sans epaisseur avec nappes tourbillonnaires evolutives. La Recherche Aérospatiale 1973;2:53-61.

[107] Mudry A. La theorie generale des nappes et filaments tourbillonnaires et ses applications a l'aerodynamique instationnaire. PhD thesis. Paris VI; 1982.

[108] Knio O, Ghoniem A. Numerical study of three-dimensional vortex methods. Journal of Computational Physics 1990;86:75-106, http://dx.doi.org/ 10.1016/0021-9991(90)90092-F.

[109] Bogateanu R, Frunzulică F, Cardos V. Unsteady free-wake vortex particle model for HAWT. In: American institute of physics conference proceedings. vol. 1281. 2010. p. 1855-8 http://dx.doi.org/10.1063/1.3498265. 
[110] Commerford GL, Carta FO. Unsteady aerodynamic response of a twodimensional airfoil at high reduced frequency. AIAA Journal 1974;12(1):43-8, http://dx.doi.org/10.2514/3.49151.

[111] Archibald FS. Unsteady Kutta condition at high values of the reduced frequency parameter. Journal of Aircraft 1975;12(6):545-50, http://dx.doi.org/10.2514/ 3.44472.

[112] Satyanarayana B, Davis S. Experimental studies of unsteady trailing-edge conditions. AIAA Journal 1978;16(2):125-9, http://dx.doi.org/10.2514/ 3.60869 .

[113] Basu BC, Hancock GJ. The unsteady motion of a two-dimensional aerofoil in incompressible inviscid flow. Journal of Fluid Mechanics 1978;87:159-78, h ttp://dx.doi.org/10.1017/S0022112078002980.

[114] Fleeter S. Trailing edge conditions for unsteady flows at high reduced frequency. AIAA Journal 1980;18(5):497-503, http://dx.doi.org/10.2514/ 3.50783 .

[115] Katz J, Weihs D. Wake rollup and the Kutta condition for airfoils oscillating at high frequency. AIAA Journal 1981;19(12):1604-6, http://dx.doi.org/ $10.2514 / 3.60097$.

[116] Poling DR, Telionis DP. The response of airfoils to periodic disturbancesthe unsteady Kutta condition. AIAA Journal 1986;24(2):193-9, http://dx.doi.org/ $10.2514 / 3.9244$

[117] Karamcheti K. Principles of ideal-fluid aerodynamics. John Wiley \& Sons Inc.; 1966.

[118] Moran J. An introduction to theoretical and computational aerodynamics. John Wiley \& Sons Inc.; 1984

[119] Anderson JD. Fundamentals of aerodynamics, 3rd ed. Aeronautical and aerospace engineering Series. McGraw-Hill; 2001.

[120] Margason RJ, Kjerlgaard SO, Sellers WL, Morris Jr. CEK, Walkey KB, Shields EW. Subsonic panel methods-a comparison of several production codes. AIAA-85-0280; January 1985.

[121] Hess JL, Smith AMO. Calculation of non-lifting potential flow about arbitrary three-dimensional bodies. Technical report ES-40622. Douglas Aircraft Co. Long Beach, CA, USA; March 1962.

[122] Karkehabadi R. Thick wings in steady and unsteady flows. Journal of Aircraft 2004;41(4):964-7, http://dx.doi.org/10.2514/1.4589.

[123] Ahmadi AR, Widnall SE. Unsteady lifting-line theory as a singular perturbation problem. Journal of Fluid Mechanics 1985;153:59-81, http://dx.doi.org $10.1017 /$ S0022112085001148.

[124] Van Dyke MD. Perturbation methods in fluid mechanics. Parabolic Press: 1975.

[125] Wilmott P. Unsteady lifting-line theory by the method of matched asymptotic expansions. Journal of Fluid Mechanics 1988;186:303-20, http://dx.do i.org/10.1017/S0022112088000151.

[126] Willis DJ, Persson P-O, Israeli ER, Peraire J, Swartz SM, Breuer KS. A computational framework for fluid structure interaction in biologically inspired flapping flight. In: AIAA applied aerodynamics conference. AIAA 2007-3803, Miami, FL, USA; 2007.

[127] Wagner H. Über die Entstehung des dynamischen Auftriebes von Tragflügeln Zietschrift für Angewandte Mathematik und Mechanick 1925;5(1):17.

[128] Küssner HG. Zusammenfassender Bericht über den instationären Auftrieb von Flügeln. Luftfahrtforschung 1936;13(12):410-24.

[129] Theodorsen T. General theory of aerodynamic instability and the mechanism of flutter. NACA report no. 496; 1935.

[130] Sears WR. Some aspects of non-stationary airfoil theory and its practical application. Journal of the Aeronautical Sciences 1941;8(5):104-8.

[131] Leishman JG, Nguyen KQ. State-space representation of unsteady airfoil behaviour. AIAA Journal 1989;28(5):836-44, http://dx.doi.org/10.2514/ 3.25127.

[132] Peters DA, Karunamoorthy S, Cao W. Finite state induced flow models. Part I: two-dimensional thin airfoil. Journal of Aircraft 1995;32(2):313-22, http://dx.doi.org/10.2514/3.46718.

[133] Peters DA. Two-dimensional incompressible unsteady airfoil theory-an overview. Journal of Fluids and Structures 2008;24:295-312, http://dx.doi.org 10.1016/j.jfluidstructs.2007.09.001.

[134] Cook R, Palacios R, Goulart P. Robust gust alleviation and stabilization of very flexible aircraft. AIAA Journal, in press.

[135] Leishman JG, Beddoes TS. A semi-empirical model for dynamic stall. Journal of American Helicopter Society 1989;34(3):3-17, http://dx.doi.org/10.4050 JAHS.34.3.

[136] Petot D. Modélisation du décrochage dynamique. La Recherche Aérospatiale 1995;5:60-72.

[137] Drela M, Youngren H. XFOIL 6.9 user primer <http://web.mit.edu/drela/ Public/web/xfoil/ > Last retrieved: 15 January 2012; 2001.

[138] Hepperle M. JAVAFOIL user's guide 〈http://www.mh-aerotools.de/airfoils/ javafoil.htm > Last retrieved: 15 January 2012; 2010

[139] Blair M. A compilation of the mathematics leading to the doublet-lattice method. USAF WL-TR-95-3022; 1994.

[140] Rodden WP. The development of the doublet-lattice method. In: International forum on aeroelasticity and structural dynamics, Rome, Italy; 1997.

[141] Cavagna L, Ricci S, Travaglini L. Aeroelastic analysis and optimization at conceptual design level using NeoCASS suite. In: $52^{\text {nd }}$ AIAA/ASME/ASCE AHS/ASC structures, structural dynamics, and materials conference. AIAA 2005-2079, Denver, CO, USA; 2011.

[142] van Zyl LH. Aeroelastic analysis of T-tails using an enhanced doublet lattice method. Journal of Aircraft 2011;48(3):823-31, http://dx.doi.org/10.2514/ 1.C001000.
[143] MSC Software, MD Nastran 2011 \& MSC Nastran 2011 release guide $\langle$ http://www.mscsoftware.com/〉. Last retrieved: 15 January 2012.

[144] ZONA Technology, Inc., Scottsdale, AZ, USA, ZAERO Version 8.3, user's manual 〈http://www.zonatech.com/ZAERO.htm〉 Last retrieved: 15 January 2012.

[145] Mukherjee R, Gopalarathnam A. Post-stall prediction of multiple-liftingsurface configurations using a decambering approach. Journal of Aircraft 2006;43(3):660-8, http://dx.doi.org/10.2514/1.15149.

[146] Gogulapati A, Friedmann PP. Approximate aerodynamic and aeroelastic modeling of flapping wings in hover and forward flight. In: $52^{\text {nd }}$ AIAA/ASME/ASCE/AHS/ASC structures, structural dynamics, and materials conference. AIAA 2011-2008, Denver, CO, USA; 2011.

[147] Patil MJ, Hodges DH. Flight dynamics of highly flexible flying wings. Journal of Aircraft 2006;43(6):1790-8, http://dx.doi.org/10.2514/1.17640.

[148] Vepa R. Finite-state modeling of aeroelastic systems. NASA CR-2779; 1977.

[149] Karpel M. Design for active flutter suppression and gust alleviation using state space aeroelastic modeling. Journal of Aircraft 1982;19(3):221-7. http://dx.doi.org/10.2514/3.57379.

[150] Karpel M. Procedures and models for aeroservoelastic analysis and design ZAMM-Journal of Applied Mathematics and Mechanics/Zeitschrift für Angewandte Mathematik and Mechanik 2001;81(9):579-92, http://dx.doi. org/10.1002/1521-4001(200109)81:9 < 579::AID-ZAMM579> 3.0.CO;2-Z.

[151] De Gaspari A, Ricci S, Riccobene L, Scotti A. Active aeroelastic control over a multisurface wing: modeling and wind-tunnel testing. AIAA Journal 2009;47(9):1995-2010, http://dx.doi.org/10.2514/1.34649.

[152] Ashley H. Engineering analysis of flight vehicles. Addison-Wesley aerospace series, Addison-Wesley Publishing Co.; 1974.

[153] Stevens BL, Lewis FL. Aircraft control and simulation, 2nd ed. John Wiley \& Sons, Inc.; 2003.

[154] Stengel RF. Flight dynamics. Princeton University Press; 2004.

[155] Palacios R, Climent H, Karlsson A, Winzell B. Assessment of strategies for correcting linear unsteady aerodynamics using CFD or experimental results. In: Progress in computational flow-structure interaction. Springer-Verlag; 2003. p. 209-24. Presented at the IFASD conference, Madrid 2001 [Chapter 8].

[156] Voss R, Tichy L, Thormann R. A ROM based flutter prediction process and its validation with a new reference model. In: 15th international forum of aeroelasticity and structural dynamics. IFASD 2011-036, Paris, France; 2011.

[157] Roger KL. Airplane math modelling and active aeroelastic control design. Technical report. AGARD-CP-228; 1977.

[158] Eversman W, Tewari A. Consistent rational fraction approximation for unsteady aerodynamics. Journal of Aircraft 1991;28(9):545-52, http://dx.d oi.org/10.2514/3.46062.

[159] Cook MV. Flight dynamics principles: a linear systems approach to aircraft stability and control, 2nd ed. Elsevier Ltd; 2007.

[160] Ghoreyshi M, Badcock KJ, Woodgate MA. Accelerating the numerical generation of aerodynamic models for flight simulation. Journal of Aircraft 2009;46(3):972-80, http://dx.doi.org/10.2514/1.39626.

[161] Da Ronch A, Ghoreyshi M, Badcock KJ. On the generation of flight dynamics aerodynamic tables by computational fluid dynamics. Progress in Aerospace Sciences 2011;47(8):597-620, http://dx.doi.org/10.1016/j.paerosci.2011.09.001.

[162] Roskam J. Airplane flight dynamics and automatic flight control systems. DARcorporation; 1979.

[163] Wildschek A, Stroscher F, Klimmek T, Šika Z, Vampola T, Valášek M, et al. Gust load alleviation on a large blended wing body airliner. In: 27th international congress of the aeronautical sciences, Nice, France; 2010.

[164] Kier KM. An integrated loads analysis model including unsteady aerodynamic effects for position and attitude dependent gust fields. In: 15th international forum of aeroelasticity and structural dynamics. IFASD 2011-052, Paris, France; 2011.

[165] Dovgiǐ S, Shekhovtsov A. An improved vortex lattice method for nonstationary problems. Journal of Mathematical Sciences 2001;104(6): 1615-27, http://dx.doi.org/10.1023/A:1011325112413.

[166] Ashurst WT, Meiburg E. Three-dimensional shear layers via vortex dynamics. Journal of Fluid Mechanics 1988;189:87-116, http://dx.doi.org/ $10.1017 /$ S0022112088000928.

[167] Lighthill MJ. A new approach to thin airfoil theory. The Aeronautical Quarterly 1951;3:193-210.

[168] Murua J. Flexible-aircraft dynamics with a geometricallly-nonlinear description of the unsteady aerodynamics. PhD thesis. Imperial College London; 2012.

[169] Silva WA, Bartels RE. Development of reduced-order models for aeroelastic analysis and flutter prediction using the CFL3Dv6.0 code. Journal of Fluids and Structures 2004;19(6):729-45, http://dx.doi.org/10.1016/j.jfluidstructs. 2004.03.004.

[170] Shabana AA. Flexible multibody dynamics: review of past and recent developments. Multibody System Dynamics 1997;1(2):189-222, http://dx.doi.org/ 10.1023/A:1009773505418.

[171] Bisplinghoff RL, Ashley H. Principles of aeroelasticity. John Wiley \& Sons, Inc.; 1962. [Reprinted by Dover Publications, Inc.; 1975].

[172] Milne RD. Dynamics of the deformable aeroplane. Parts I and II. In: Aeronautical research council reports and memoranda. No. 3345; 1962.

[173] Meirovitch L, Tuzcu I. The lure of the mean axes. Journal of Applied Mechanics 2007;74:497-504, http://dx.doi.org/10.1115/1.2338060.

[174] Hodges DH. A mixed variational formulation based on exact intrinsic equations for dynamics of moving beams. International Journal of Solids 
and Structures 1990;26(11):1253-73, http://dx.doi.org/10.1016/00207683(90)90060-9.

[175] Simo JC, Vu-Quoc L. A three-dimensional finite-strain rod model. Part II: computational aspects. Computer Methods in Applied Mechanics and Engineering 1986;58:79-116, http://dx.doi.org/10.1016/0045-7825(86)90079-4.

[176] Géradin M, Cardona A. Flexible multibody dynamics: a finite element approach. John Wiley Sons, Inc.; 2001.

[177] Cesnik CES, Brown EL. Modeling of high aspect ratio active flexible wings for roll control. In: 43rd AIAA/ASME/ASCE/AHS/ASC structures, structural dynamics, and materials conference. No. AIAA 2002-1719, Denver, CO, USA; 2002.

[178] Hodges DH. Geometrically exact, intrinsic theory for dynamics of curved and twisted anisotropic beams. AIAA Journal 2003;41(6):1131-7, http://dx. doi.org/10.2514/2.2054.

[179] Sotoudeh Z, Hodges DH. Incremental method for structural analysis of joinedwing aircraft. Journal of Aircraft 2011;48(5):1588-601, http://dx.doi.org/ 10.2514/1.C031302.

[180] Palacios R. Nonlinear normal modes in an intrinsic theory of anisotropic beams. Journal of Sound and Vibration 2011;330(8):1772-92, http://dx.doi.org/ 10.1016/j.jsv.2010.10.023.

[181] Cesnik CES, Hodges DH. VABS: a new concept for composite rotor blade cross-sectional modeling. Journal of the American Helicopter Society 1997;42(1):27-38, http://dx.doi.org/10.4050/JAHS.42.27.

[182] Palacios R, Cesnik CES. Cross-sectional analysis of non-homogeneous anisotropic active slender structures. AIAA Journal 2005;43(12):2624-38, http://dx.doi.org/10.2514/1.12451.

[183] Cramer EJ, Dennis Jr JE, Frank PD, Lewis RM, Shubin GR. Problem formulation for multidisciplinary optimization. SIAM Journal on Optimization 1994;4(4):754-76, http://dx.doi.org/10.1137/0804044.

[184] Farhat C, Lesoinne M, Stern P, Lantéri S. High performance solution of threedimensional nonlinear aeroelastic problems via parallel partitioned algorithms: methodology and preliminary results. Advances in Engineering Software 1997;28:43-61, http://dx.doi.org/10.1016/S0965-9978(96)00028-2.

[185] Murua J, Palacios R, Graham J. Modeling of nonlinear flexible aircraft dynamics including free-wake effects. In: AIAA atmospheric flight mechanics conference. AIAA 2010-8226, Toronto, ON, Canada; 2010.

[186] Murua J, Hesse H, Palacios R, Graham JMR. Stability and open-loop dynamics of very flexible aircraft including free-wake effects. In: 52nd AIAA/ASME/ASCE/AHS/ASC structures, structural dynamics, and materials conference, AIAA 2011-1915, Denver, CO, USA, 2011.

[187] Murua J, Palacios R, Graham JMR. A discrete-time state-space model with wake interference for stability analysis of flexible aircraft. In: 15th international forum of aeroelasticity and structural dynamics. IFASD 2011-127, Paris, France; 2011.

[188] Baldock J. Determination of the flutter speed of a T-tail unit by calculations, model tests and flight flutter tests. Technical report. AGARD Report 221; 1958.

[189] Livne E, Weisshaar T. Aeroelasticity of nonconventional airplane configurationspast and future. Journal of Aircraft 2003;40(6):1047-65, http://dx.doi.org/10.2514/ 2.7217.

[190] Raymer DP. Aircraft design: a conceptual approach, 4th ed. AIAA Education Series. American Institute of Aeronautics and Astronautics, Inc.; 2006.
[191] Attorni A, Cavagna L, Quaranta G. Aircraft T-tail flutter predictions using computational fluid dynamics. Journal of Fluids and Structures 2011;27:161-74, http://dx.doi.org/10.1016/j.jfluidstructs.2010.11.003.

[192] McCuE DJ, Gray R, Drane DA. The effect of steady tailplane lift on the subcritical response of a subsonic T-tail flutter model. In: Aeronautical research council reports and memoranda. No. 3652; 1971.

[193] Gray R, Drane DA. The effect of steady tailplane lift on the oscillatory behaviour of a T-tail flutter model at high subsonic speeds. In: Aeronautical research council reports and memoranda. No. 3745; 1974.

[194] Jennings WP, Berry MA. Effect of stabilizer dihedral and static lift on T-tail flutter. Journal of Aircraft 1977;14(4):364-7, http://dx.doi.org/10.2514/ 3.58785 .

[195] Love MH, Zink PS, Wieselmann PA, Youngren H. Body freedom flutter of high aspect ratio flying wings. In: 46th AIAA/ASME/ASCE/AHS/ASC structures, structural dynamics, and materials conference. AIAA 2005-1947, Austin, TX, USA; 2005.

[196] Hoblit FM. Gust loads on aircraft: concepts and applications. AIAA Education Series. American Institute of Aeronautics and Astronautics, Inc.; 1988.

[197] Lomax TL. Structural loads analysis for commercial transport aircraft: theory and practice. AIAA Education Series. American Institute of Aeronautics and Astronautics, Inc.; 1996.

[198] Howe D. Aircraft loading and structural layout, aerospace series. Professional Engineering Publishing; 2004.

[199] Noll TE, Ishmael SD, Henwood B, Perez-Davis ME, Tiffany GC, Gaier M, et al. Technical findings, lessons learned, and recommendations resulting from the Helios prototype vehicle mishap. In: NATO/RTO AVT-145 workshop on design concepts, processes and criteria for uav structural integrity, Florence, Italy; 2007.

[200] Su W, Cesnik CES. Dynamic response of highly flexible flying wings. AIAA Journal 2011;49(2):324-39, http://dx.doi.org/10.2514/1.J050496.

[201] Certification Specifications, European Aviation Safety Agency.

[202] Federal Aviation Administration Regulations. Part 25-airworthiness standards: transport category airplanes. US Department of Transportation.

[203] Zole A, Karpel M. Continuous gust response and sensitivity derivatives using state-space models. Journal of Aircraft 1994;31(5):1212-4, http://dx.doi.org/ $10.2514 / 3.46632$

[204] Liepmann HW. On the application of statistical concepts to the buffeting problem. Journal of Aeronautical Sciences 1952;19(12):793-800.

[205] Diederich FW, Drischler JA. Effect of spanwise variations in gust intensity on the lift due to atmospheric turbulence. NACA TN 3920; April 1957.

[206] Houbolt JC, Steiner R, Pratt KG. Dynamic response of airplanes to atmospheric turbulence including flight data on input and response. NASA TR R-199 (initially presented as an AGARD paper, July 1962); June 1964.

[207] Campbell CW. Monte Carlo turbulence simulation using rational approximations to von Kármán spectra. AIAA Journal 1986;24(1):62-6, http://dx.doi.org/ $10.2514 / 3.9223$.

[208] Raveh DE. Gust-response analysis of free elastic aircraft in the transonic flight regime. Journal of Aircraft 2011;48(4):1204-11, http://dx.doi.org/ 10.2514/1.C031224. 